Portland State University

PDXScholar

Spring 5-19-2016

\title{
Physics-Based Imaging Methods for Terahertz Nondestructive Evaluation Applications
}

\author{
Gabriel Paul Kniffin \\ Portland State University
}

Follow this and additional works at: https://pdxscholar.library.pdx.edu/open_access_etds

Part of the Electromagnetics and Photonics Commons, and the Physics Commons Let us know how access to this document benefits you.

\section{Recommended Citation}

Kniffin, Gabriel Paul, "Physics-Based Imaging Methods for Terahertz Nondestructive Evaluation Applications" (2016). Dissertations and Theses. Paper 2945.

https://doi.org/10.15760/etd.2940

This Dissertation is brought to you for free and open access. It has been accepted for inclusion in Dissertations and Theses by an authorized administrator of PDXScholar. Please contact us if we can make this document more accessible: pdxscholar@pdx.edu. 


\title{
Physics-Based Imaging Methods
}

for Terahertz Nondestructive Evaluation Applications

by

Gabriel Paul Kniffin

A dissertation submitted in partial fulfillment of the requirements for the degree of

\author{
Doctor of Philosophy \\ in \\ Electrical and Computer Engineering
}
Dissertation Committee:
Lisa M. Zurk, Chair
T. Martin Siderius
Donald D. Duncan
Suresh Singh

Portland State University

2016 
(c) 2016 Gabriel Paul Kniffin 


\begin{abstract}
Lying between the microwave and far infrared (IR) regions, the "terahertz gap" is a relatively unexplored frequency band in the electromagnetic spectrum that exhibits a unique combination of properties from its neighbors. Like in IR, many materials have characteristic absorption spectra in the terahertz ( $\mathrm{THz})$ band, facilitating the spectroscopic "fingerprinting" of compounds such as drugs and explosives. In addition, non-polar dielectric materials such as clothing, paper, and plastic are transparent to $\mathrm{THz}$, just as they are to microwaves and millimeter waves. These factors, combined with sub-millimeter wavelengths and non-ionizing energy levels, makes sensing in the $\mathrm{THz}$ band uniquely suited for many NDE applications.
\end{abstract}

In a typical nondestructive test, the objective is to detect a feature of interest within the object and provide an accurate estimate of some geometrical property of the feature. Notable examples include the thickness of a pharmaceutical tablet coating layer or the 3D location, size, and shape of a flaw or defect in an integrated circuit. While the material properties of the object under test are often tightly controlled and are generally known a priori, many objects of interest exhibit irregular surface topographies such as varying degrees of curvature over the extent of their surfaces. Common THz pulsed imaging (TPI) methods originally developed for objects with planar surfaces have been adapted for objects with curved surfaces through use of mechanical scanning procedures in which measurements are taken at normal incidence over the extent of the surface [1]. While effective, these methods often require expensive robotic arm assemblies, the cost and complexity of which would likely be prohibitive should a large volume of tests be needed to be carried out on a production line. 
This work presents a robust and efficient physics-based image processing approach based on the mature field of parabolic equation methods, common to undersea acoustics, seismology, and other areas of science and engineering. The method allows the generation of accurate 3D THz tomographic images of objects with irregular, non-planar surfaces using a simple planar scan geometry, thereby facilitating the integration of 3D THz imaging into mainstream NDE use.

The key contributions of this work are:

- The development of a parabolic equation-based processing method for focusing terahertz $(\mathrm{THz})$ phased array data through objects with irregular surface topographies, including varying degrees of curvature. Parabolic equation (PE) methods have been used for decades by the seismology, underwater acoustics, and radar communities to model electromagnetic and acoustic wave propagation. Despite their long history, PE methods have only recently been applied to imaging problems in ground-penetrating radar and medical ultrasound. This work constitutes the very first application of PE methods to THz imaging.

- The design and implementation terahertz tomographic imaging experiments conducted using the Picometrix T-Ray 4000 pulsed THz time-domain imaging system at the Northwest Electromagnetics and Acoustics Research Laboratory (NEAR-Lab) as an experimental testbed - to demonstrate the method's ability to correct for refraction through curved dielectric boundaries and accurately localize features of interest within the sample under test. Such features include boundaries between curved dielectric layers, allowing the quantification of their thickness.

- The design and implementation of numerical test simulations to quantify the 
influence of errors due to approximations made in the method's derivation on the accuracy of the resulting 3D tomographic images.

- The implementation and numerical validation of an analytic, Mie series model for electromagnetic scattering from a layered dielectric sphere. 


\section{Acknowledgments}

Science is a cooperative enterprise, spanning the generations.

It's the passing of a torch from teacher to student to teacher.

A community of minds reaching back from antiquity and forward to the stars.

NeIL DeGrasse Tyson

To all of the teachers and mentors I've had over the years, I thank you for helping me in my journey and for making me a better human being. You are the giants on whose shoulders I stand. In particular, I am deeply thankful to my advisor, Dr. Lisa Zurk, for her guidance and advocacy, and for believing in me when I needed it most. The same applies to Dr. Martin Siderius, whose constant encouragement, patience, and mentorship have been invaluable to me throughout my time at the NEAR-Lab. Thanks are also due to Dr. Don Duncan for his support and for introducing me to the subjects of Fourier and statistical optics, the intuition I gained from which proved extremely useful throughout this project (after the material finally sunk in). I'd also like to thank Dr. Suresh Singh for expanding my horizons and for trusting in my abilities enough to let me assist him in some of his own work. I'm extremely grateful to the four of you for all you've done for me in addition to serving on my Ph.D. committee. I'm also thankful to Dr. Rick Campbell, who has always had a 
knack for igniting my curiosity, and Dr. Dan Rouseff for his support and for sharing his expertise and insights on subjects pertinent to my own work. Thanks also to Dr. Branimir Pejcinovic and Dr. Alla Timchenko for their support.

And of course, I couldn't have made it this far without the support and friendship of my peers in the NEAR-Lab over the years: Scott Schecklman, Sam Henry, John Gebbie, Elizabeth Küsel, Lanfranco Muzi, Dan Frister, John Boyle, Reid McCargar, Richard Campbell, Jimmy Gladish, Nathan Laws, Eran Schweitzer, Pasang Sherpa, Jordan Shibley, Jorge and Zizheng Quijano, Alex Higgins, Forest Kernan, George Ogden, Eric Sorensen, Don Tornquist, Joel Paddock, Tobias Oesterlein, Kyung Nam, Garth Sundberg, and Shari Matzner. Thanks for being awesome.

Finally, I'd like to acknowledge and thank the National Science Foundation for helping me launch my career by sponsoring this research. 


\section{Table of Contents}

Abstract $\quad$ i

Acknowledgments $\quad$ iv

List of Figures $\quad$ ix

Chapter 1 Introduction and Motivation 1

1.1 Contributions and Significance . . . . . . . . . . . . 7

1.2 Executive Summary . . . . . . . . . . . . . . . . 9

$\begin{array}{lll}\text { Chapter } 2 & \text { Literature Review } & 13\end{array}$

2.1 Terahertz Non-Destructive Evaluation . . . . . . . . . . . . 13

2.2 3D Terahertz Imaging . . . . . . . . . . . . . . . . 15

2.3 Wave Propagation Methods . . . . . . . . . . . . . . . . . . . 19 
2.4 Mie Series Solution for Layered Sphere Scattering . . . . . . . . . . . 20

\section{$\begin{array}{lll}\text { Chapter } 3 & \text { Theoretical Background } & 23\end{array}$}

3.1 Reflection Mode Diffraction Tomography . . . . . . . . . . . . . 23

3.2 Parabolic Equation Methods . . . . . . . . . . . . . . . . . . 33

3.2.1 Fourier Back-Propagation ................ 35

Broadband Imaging Resolution: A Geometrical Interpretation 37

3.2.2 Split-Step Fourier Back-Propagation . . . . . . . . . . . . . . 42

Implementation . . . . . . . . . . . . . . . . . 44

Error Analysis: Propagation Step Size . . . . . . . . . . 45

Error Analysis: Propagation Angle and Refractive Index Per-

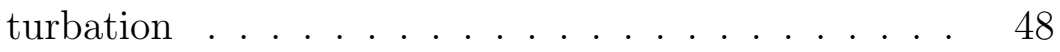

Chapter 4 Implementation, Demonstration, and Analysis 51

4.1 Picometrix T-Ray 4000 Imaging System . . . . . . . . . . . . . . 52

4.1.1 Waveform Preprocessing . . . . . . . . . . . . 55

4.2 Experimental Results . . . . . . . . . . . . . . . . . 61

4.2.1 Pharmaceutical Tablet ............... 62 
4.2.2 High-Density Polyethylene Test Object . . . . . . . . . . . . . 65

4.2 .3 3D-Printed Test Object . . . . . . . . . . . . . . 68

4.3 Error Analysis Simulations . . . . . . . . . . . . . . . . 78

$\begin{array}{lll}\text { Chapter } 5 & \text { Conclusions and Future Work } & 86\end{array}$

5.1 Broader Impacts and Future Work . . . . . . . . . . . . . . . 87

5.2 Publications . . . . . . . . . . . . . . . . . 88

$\begin{array}{ll}\text { Bibliography } & 91\end{array}$

Appendix A Mie Series Model for Scattering from a Layered Dielec$\begin{array}{ll}\text { tric Sphere } & 110\end{array}$

A.1 Mie Series Model Derivation . . . . . . . . . . . . . . . . 110

A.2 Mie Series Model Validation . . . . . . . . . . . . . . . 117 


\section{List of Figures}

1.1 The "Terahertz Gap" shown within the greater electromagnetic spectrum. 1

1.2 (a) Photograph of 8-pin integrated circuit (IC) measured by the author using a $\mathrm{THz}$ imaging system to be described in a later section. (b) Top-down THz image (C-scan) of IC. (c) Cross-sectional slice (B-scan) image taken along horizontal line in $\mathrm{THz}$ C-scan. (d) THz time-domain waveforms (A-scans) taken along vertical lines in $\mathrm{THz} \mathrm{B}$-scan. . . . . .

1.3 Example of refractive distortion. The subject's feet appear significantly displaced from their actual location due to the oblique viewing angle from the surfaces of the water tank. . . . . . . . . . . 5

1.4 Pharmaceutical tablets exhibit convex surfaces with layers of coating. $\quad 6$

1.5 Diagram of possible $\mathrm{THz}$ phased array imaging configurations using either a physical (a) or (inverse) synthetic (b) phased array. . . . . . . 7

3.1.1 Imaging geometry assumed in $\mathrm{THz} \mathrm{SA}$ tomography formulation. The object under test, represented by a closed region of refractive index $n(\mathbf{r})>1$, is illuminated by an isotropic point transceiver at $\mathbf{r}_{0}=$

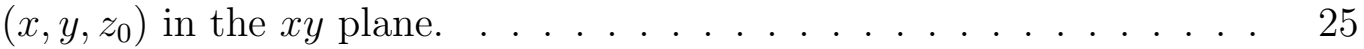

3.1.2 Wave vector shown in terms of spherical coordinates. . . . . . . . . 30

3.1.3 Illustration of the Ewald hemisphere given by the support of (3.1.26) over which the 3D Fourier transform of the scattering potential is sampled. 33

3.2.1 Image of a point scatterer at $\left(x^{\prime}, z^{\prime}\right)=(4 \mathrm{~mm}, 15 \mathrm{~mm})$ from 2D simulation data. (a) shows the resulting image along with annular arcs of radial thickness $\delta r=0.41 \mathrm{~mm}$ extending from elements of the array to the location of the scatterer. The rectangle around the scatterer indicates the bounds of the zoomed image (b) which shows the point spread function (PSF) centered at the scatterer's location. The four points bounding the PSF correspond to the intersection points of the annuli from the outermost array elements. . . . . . . . . . .

3.2.2 Axial (a) and lateral (b) resolution calculated for the simulation space in Fig. 3.2.1 using expressions (3.2.12) and (3.2.17). The red " $\times$ " indicates the location of the scatterer in Fig. 3.2.1 in the parameter space. . . . 
3.2.3 Diagram illustrating the tangent plane approximation, under which a sensor at $x_{0}$ in the array plane will receive a specular reflection from the curved surface at an angle $\theta_{p}$ from the $z$-axis, which is equal to the tilt angle of the tangent plane. . . . . . . . . . . . . . .

3.2.4 Relative error in the SSF propagator (3.2.39) as a function of propagation angle $\theta_{p}$ and relative refractive index perturbation $\delta n / n_{0} \ldots \ldots$.

4.1.1 Picometrix T-Ray $4000 \mathrm{THz}$ time-domain imaging system. . . . . . .

4.1.2 Example waveforms and amplitude spectra generated using T-Ray 4000 operating in monostatic reflection mode with a $1^{\prime \prime}$ focal length lens. Waveforms shown in (a) are the coherent average of 100 and 10,000 waveforms. Coherent averaging in this way increases signal-to-noise ratio. This is more evident in (b), which shows the corresponding Fourier amplitude spectra on a log scale. As the figure shows, the noise floor is relatively flat across the $\mathrm{THz}$ spectrum. . . . . . . . . . . .

4.1.3 (a) Point-like virtual transceiver at focal point of $1^{\prime \prime}$ lens [91]. (b) Synthetic array formed by raster scanning virtual transceiver laterally in the $x y$-plane $[23,24] . \ldots \ldots \ldots \ldots$

4.1.4 Comparison of the amplitude response of the matched filter $b_{\mathrm{MF}}(\omega)$ from (4.1.3), the deconvolution filter $b_{\text {deconv. }}(\omega)$ from (4.1.7), the Wiener deconvolution filter $b_{\mathrm{WD}}(\omega)$ from (4.1.6) and the parameterized Wiener deconvolution filter $b_{\mathrm{PD}}(\omega)$ in (4.1.14), where $w_{r}(\omega)$ is given by the 10,000 reference waveform average in Fig. 4.1.2. For $b_{\mathrm{PD}}(\omega), \alpha=100$ and the power cutoff level was set to the noise floor in the 100-waveform

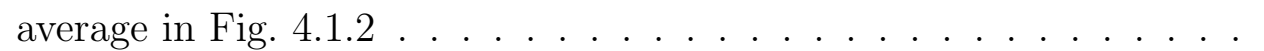

4.1.5 Comparison of the normalized output of (4.1.15), whose impulse responses correspond to the transfer functions are shown in Fig. 4.1.4. .

4.2.1 Over-the-counter gelcap tablet imaged using the Picometrix T-Ray 4000 imaging system described in Sec. 4.1. Emptying the gelcap of its liquid contents was found to enhance the $\mathrm{THz}$ reflection from the inner surface. 62

4.2.2 Axial slices through the FBP-processed (a) and SSF-processed (b) 3D image data along with the depths of the top boundary and inner boundaries. . . . . . . . . . . . . . . .

4.2.3 HDPE test object with cylindrically-curved top surface and protruding copper wires A, B, and C. (a) Diagram of test object. (b) $x z$-view showing curved top surface. (c) yz-view showing protruding copper wires. . . . . . . . . . . . . . . . . .

4.2.4 Volume rendering of $\mathrm{THz}$ SA tomographic data of HDPE test object obtained using FBP shown at 3 different angles. Returns from the curved HDPE surface and wires A, B, and C (see Fig. 4.2.3) are labeled. 67 
4.2.5 Volume rendering of $\mathrm{THz} \mathrm{SA}$ tomographic data of HDPE test object obtained using SSF back-propagation shown at 3 different angles. Returns from the curved HDPE surface and wires A, B, and C are labeled. 69

4.2.6 Side-by-side comparison of cross-sectional slices of THz SA tomographic data obtained using (a) FBP and (b) SSF back-propagation. Slices are shown at $x=-3.65 \mathrm{~mm},-0.45 \mathrm{~mm}$, and $2.75 \mathrm{~mm}$, corresponding to wires A, B, and C, respectively. Returns from the HDPE surface are labeled and arrows indicate the downward shift of the internal wires from their correct location (dotted lines) due to refractive effects. The image distortions introduced by the HDPE have been largely corrected by the SSF method, as indicated by the continuity of the bright returns from the protruding ends of the wires and the segments of the wires within the HDPE. . . . . . . . . . . . . . . . .

4.2.7 (a) Diagram of 3D printed hemicylindrical test object with $5 \mathrm{~mm}$ outer radius and axially-sloped inner radius, ranging from $4 \mathrm{~mm}$ on one end to $3.5 \mathrm{~mm}$ on the other. Copper tape was affixed to the inside of the sample to provide a high-contrast return as shown in the photographs (b) and (c). . . . . . . . . . . . . . . . . . .

4.2.8 Cross-sectional slice images through 3D THz tomographic images produced using FBP (a) and SSF (b) methods at the same $y$ value. The top boundary $z_{\text {top }}\left(x, y_{\text {slice }}\right)$ and inner boundaries, estimated from the depth of the protruding ends $z_{\text {inner }}\left(x, y_{\text {slice }}\right)$ are visible in both images as dashed blue and solid orange lines, respectively. In the FBP-processed data (a), the inner surface $z_{\mathrm{FBP}}\left(x, y_{\text {slice }}\right)$ (dotted purple line) appears displaced vertically from $z_{\text {inner }}\left(x, y_{\text {slice }}\right)$ due to refraction. In the SSFprocessed data (b), the refraction is corrected, bringing the inner surface $z_{\text {SSF }}\left(x, y_{\text {slice }}\right)$ (dot-dashed green line) into alignment with $z_{\text {inner }}\left(x, y_{\text {slice }}\right) .73$

4.2.9 Layer thickness estimates calculated using (4.2.1)-(4.2.3) for the image data in Fig. 4.2.8. . . . . . . . . . . . . . . . 76

4.2.10 Contour plots of layer thickness error in millimeters. (a) Layer thickness error from FBP-processed data calculated using (4.2.4). (b) Layer thickness error from SSF-processed data calculated using (4.2.5). While some error remains, SSF processing significantly reduces thickness error. 77

4.3.1 Relative error in the SSF propagator (3.2.39) as a function of propagation angle $\theta_{p}$ and relative refractive index perturbation $\delta n / n_{0}$ along with lines representing where the error in the data set presented in Sec. 4.2 .3 falls. . . . . . . . . . . . . . . . . . . 80

4.3.2 Diagram of tilted dielectric layer assumed in simulations. . . . . . . 81 
4.3.3 Imaging results from simulated data from tilted dielectric slab shown in Fig. 4.3.2 for the case of $\theta_{p}=32^{\circ}$, shown with $z_{\text {top }}\left(x, \theta_{p}\right)$ and $z_{\text {bottom }}\left(x, \theta_{p}\right)$ for reference. (a) Results of FBP processing, which doesn't correct for refraction within the object. (b) Results of SSF processing given the layer's $\mathrm{THz}$ refractive index $n=1.59 \ldots \ldots \ldots \ldots$

4.3.4 Thickness error for a range of tilt angles for both FBP and SSF processed data. . . . . . . . . . . . . . . . . . . . . 


\section{Chapter 1}

\section{Introduction and Motivation}

The "terahertz gap" lies between the microwave and infrared bands in the electromagnetic spectrum, shown in Fig. 1.1. Historically, the development of efficient (THz) sources and receivers has lagged behind that of the neighboring microwave and infrared bands. However, recent advances in both microwave/RF and optical technologies have begun to fill in the gap, unlocking a host of new and potentially revolutionary technologies and applications in a wide variety of scientific and engineering disciplines.

The growing interest in $\mathrm{THz}$ technology is largely due to the unique combination of properties exhibited by $\mathrm{THz}$ radiation. Like in the infrared band, many materials have unique absorption spectra in the $\mathrm{THz}$ band, facilitating the spectroscopic fin-

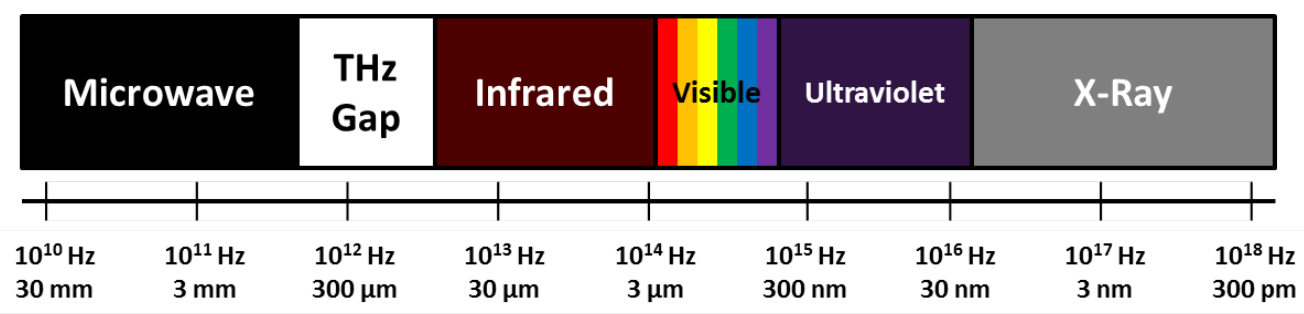

Figure 1.1: The "Terahertz Gap" shown within the greater electromagnetic spectrum. 
gerprinting of compounds such as drugs and explosives [2, 3]. In addition, non-polar materials such as clothing, paper, and plastic are transparent to $\mathrm{THz}$, just as they are to microwaves and millimeter waves. The relatively short wavelengths of $\mathrm{THz}$ waves (0.1-1 mm) and broadband nature of many common $\mathrm{THz}$ systems also makes $\mathrm{THz}$ technology attractive for imaging applications. These properties, combined with the low (non-ionizing) energy levels of $\mathrm{THz}$ waves drove much of the early interest in $\mathrm{THz}$ sensing for security screenings of mail or luggage and passengers in airports. However, these features also make THz waves uniquely suited for many non-destructive evaluation (NDE) applications.

In most of these applications, an image of the internal structure of the object under test is desired in order to detect the presence and determine the physical characteristics of a feature of interest within the object. Real-world examples of such features include pharmaceutical tablet coatings $[4,5]$, automotive paint layers $[6,7]$, as well as faults or defects in polymer parts [8-10], integrated circuits [11-13], and aerospace components [14-16].

A common approach to such THz NDE problems is THz pulsed imaging (TPI), also known as $\mathrm{THz}$ time-of-flight (ToF) tomography [1, 17-22]. This process involves focusing broadband $\mathrm{THz}$ pulses onto a section of the sample under test and coherently detecting the reflected pulses in a manner similar to pulse-echo ultrasound [18, $20,21]$. The delay times of the returned echo pulses are then used to determine the optical distances between dielectric interfaces along the beam path. This method is illustrated in Fig. 1.2, which shows THz TPI data of an 8-pin integrated circuit (IC) measured by the author using a THz imaging system described in Chapter 4 . Fig. $1.2 \mathrm{~b}$ shows a top-down $\mathrm{THz}$ image, commonly referred to as a C-scan by the 


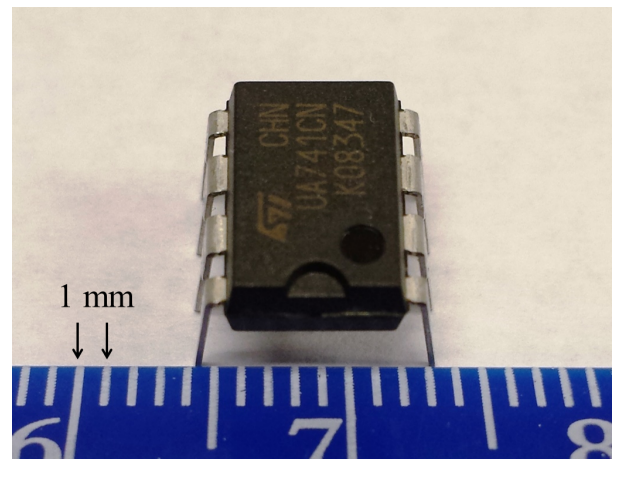

(a)

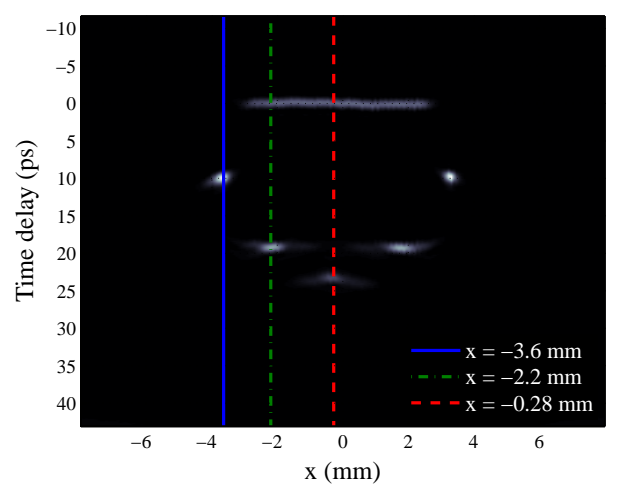

(c)

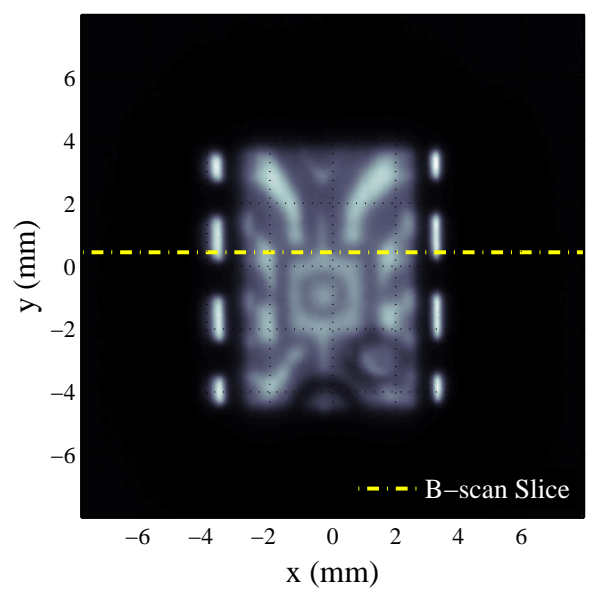

(b)

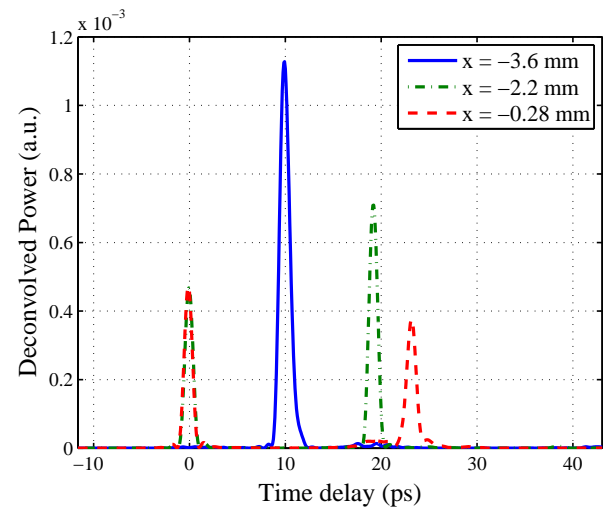

(d)

Figure 1.2: (a) Photograph of 8-pin integrated circuit (IC) measured by the author using a $\mathrm{THz}$ imaging system to be described in a later section. (b) Top-down $\mathrm{THz}$ image (C-scan) of IC. (c) Cross-sectional slice (B-scan) image taken along horizontal line in $\mathrm{THz} \mathrm{C}$-scan. (d) $\mathrm{THz}$ time-domain waveforms (A-scans) taken along vertical lines in $\mathrm{THz}$ B-scan. 
medical ultrasound community. The C-scan clearly shows the 8 metal pins as well as the IC's internal electrical contacts. The THz data in Fig. 1.2 actually represents a 3D cube of data, with each pixel in the C-scan representing a time domain waveform. This allows viewing cross-sectional slices (B-scans) through the data, such as that in Fig. 1.2c, taken along the horizontal yellow line shown in Fig. 1.2b. The B-scan shows reflections from the front surface of the IC's dielectric surface, followed by subsequent echoes from the IC's pins and conductive internal structures. Fig. 1.2d shows three waveforms (A-scans), each individual columns of the B-scan data taken along the vertical lines in Fig. 1.2c.

One limitation of TPI is that the returned echo pulses are assumed to originate from a point along the optical axis of the focusing lens. This doesn't typically cause problems for TPI of objects with approximately planar surfaces such as the IC chip in Fig. 1.2, provided the monostatic measurements are taken at normal incidence to the object's surface. In this case, refractive effects due to the dielectric material simply delay the arrival of the echo pulses, making them appear later in time. This is shown clearly in Figs. 1.2c and 1.2d, where the returns from the IC's metal pins from within the dielectric packaging at $x=-2.2 \mathrm{~mm}$ appear displaced vertically from their positions outside the dielectric packaging at $x=-3.6 \mathrm{~mm}$. Correcting for these refractive delays is a relatively straightforward process of scaling the time axis to account for the slower propagation velocity of the $\mathrm{THz}$ pluses. However, if the measurement is taken at an oblique incidence, refraction will cause the beam path to be bent away from the optical axis, resulting in distortions in the final image of the object's internal structure as illustrated in Fig. 1.3.

Furthermore, many objects of interest may present irregular surface topographies 


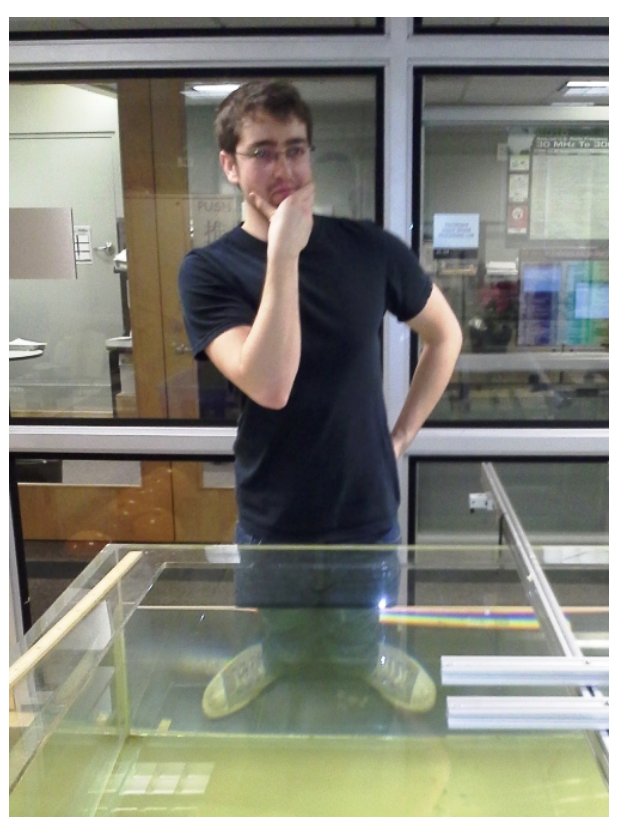

Figure 1.3: Example of refractive distortion. The subject's feet appear significantly displaced from their actual location due to the oblique viewing angle from the surfaces of the water tank.

with varying degrees of curvature or other features, which makes measurements at normal incidence to the surface much more complicated. A prime example of this arises in the NDE of pharmaceutical tablet coatings. In this case, the thickness of the coating layers is sought and typically obtained using TPI [1, 19]. However, the tablets themselves have curved outer surfaces as shown in Fig. 1.4. In order to maintain normal alignment throughout the TPI measurement process, the pioneering work by Zeitler et al. [1] employed a six-axis robotic arm to physically rotate and translate the pill throughout the scanning process. Each side of the pill is scanned in two steps, the first being the use of a laser profilometer to acquire the 3D spatial location of the pill's outermost surface, the second being a set of TPI measurements over the extent of the pill's surface to obtain a qualitative image of the layers of tablet coating. Echo pulse delay times in the TPI data are scaled by a known or assumed refractive index to provide an accurate estimate of the layer thicknesses. The pill is 

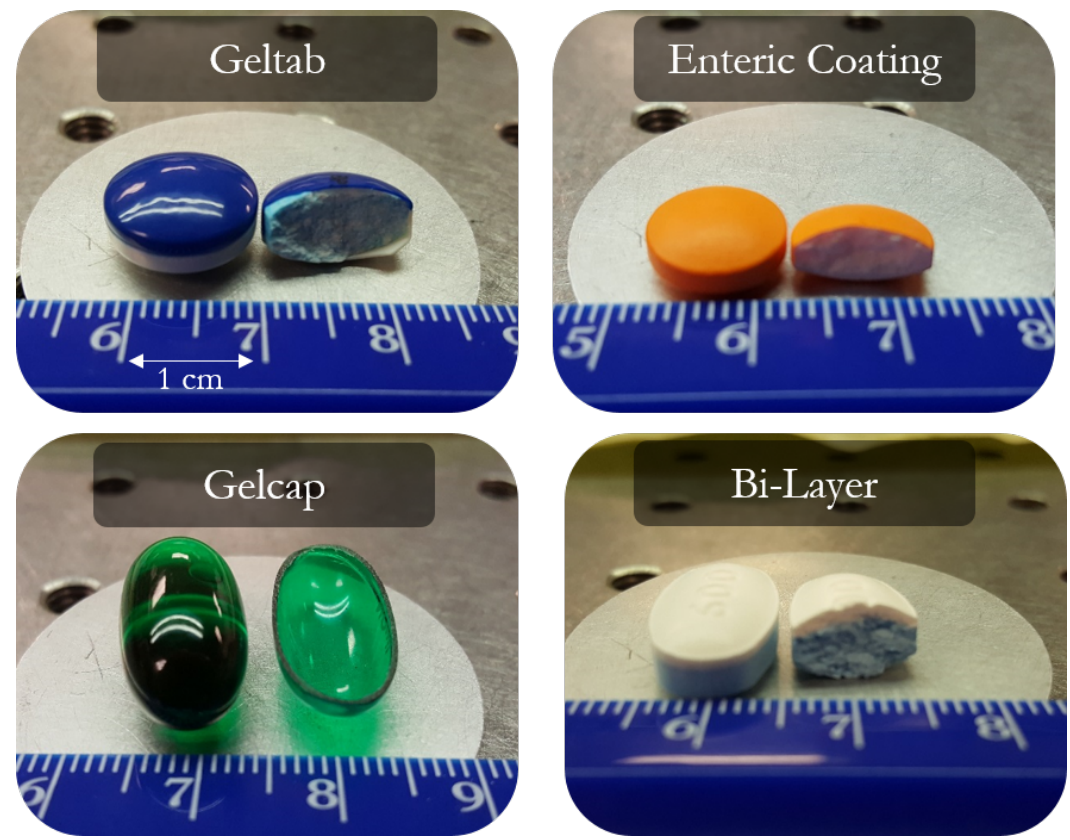

Figure 1.4: Pharmaceutical tablets exhibit convex surfaces with layers of coating.

then flipped so the other side can be measured in an identical fashion, resulting in a total of 40-60 minutes of scan time per pill. While effective, this method requires a complicated and expensive setup and would be difficult to scale up should a large volume of measurements need to be carried out on a production line.

Phased array techniques, such as THz synthetic aperture (SA) tomography [23, 24], offer an alternative with several advantages over conventional TPI. Phased array techniques allow a high degree of depth resolution without sacrificing spectral information and enable dynamic focusing to multiple depths using a single data set [25]. In addition, as $\mathrm{THz}$ technology matures, transmitter and receiver arrays are expected to decrease in cost and size while increasing in efficiency [26-28], enabling practical deployment on the production line. Two example configurations are depicted in Fig. 1.5, in which pharmaceutical tablets (or other objects under test) are imaged as they move down a conveyor belt either by a $2 \mathrm{D}$ physical planar array or by a 

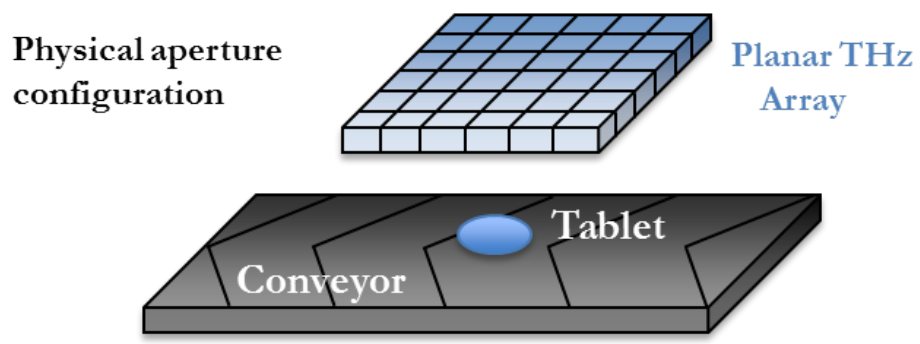

(a)

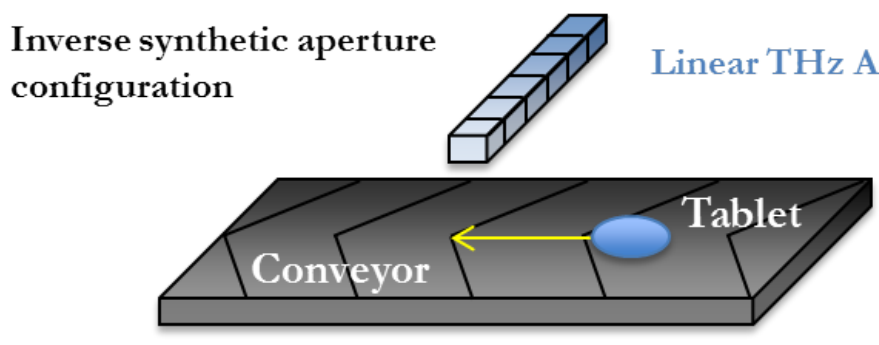

(b)

Figure 1.5: Diagram of possible $\mathrm{THz}$ phased array imaging configurations using either a physical (a) or (inverse) synthetic (b) phased array.

1D linear array operating in an inverse synthetic aperture configuration as the pills are conveyed beneath. While $3 \mathrm{D} \mathrm{THz} \mathrm{SA}$ tomographic imaging has already been demonstrated on objects with planar boundaries [23, 24], the more general case of objects with non-planar boundaries remains a significant barrier that will need to be overcome before such methods can be integrated into standard practice in the field of NDE.

\subsection{Contributions and Significance}

This dissertation advances the capabilities of THz 3D imaging technology by developing an efficient, physics-based image processing method capable of correcting for refractive effects introduced in $3 \mathrm{D} \mathrm{THz}$ tomographic images of transparent objects with irregular boundaries. Though the original motivation for this work was to 
allow focusing to within the curved layers of pharmaceutical tablets in order to nondestructively estimate the thickness of the various enteric coating layers, the method developed is much more broadly useful and is applicable to a far larger set of $\mathrm{THz}$ imaging scenarios in which accurate 3D images of a transparent object's internal structure are sought.

The key contributions of this work are:

- The development of a parabolic equation-based processing method for focusing terahertz $(\mathrm{THz})$ phased array data through objects with irregular surface topographies, including varying degrees of curvature. Parabolic equation (PE) methods have been used for decades by the seismology [29, 30], underwater acoustics [31, 32], and radar communities [33, 34] to model electromagnetic and acoustic wave propagation. Despite their long history, PE methods have only recently been applied to imaging problems in ground-penetrating radar [35, 36] and medical ultrasound $[37,38]$. This work [39-41] constitutes the very first application of $\mathrm{PE}$ methods to $\mathrm{THz}$ imaging.

- The design and implementation terahertz tomographic imaging experiments conducted using the Picometrix T-Ray 4000 pulsed THz time-domain imaging system at the Northwest Electromagnetics and Acoustics Research Laboratory (NEAR-Lab) as an experimental testbed - to demonstrate the method's ability to correct for refraction through curved dielectric boundaries and accurately localize features of interest within the sample under test. Such features include boundaries between curved dielectric layers, allowing the quantification of their thickness.

- The design and implementation of numerical test simulations to quantify the 
influence of errors due to approximations made in the method's derivation on the accuracy of the resulting 3D tomographic images.

- The implementation and numerical validation of an analytic, Mie series model for electromagnetic scattering from a layered dielectric sphere.

This work represents a novel combination of $\mathrm{THz}$ technology, phased array imaging, reflection-mode diffraction tomography, and PE methods. The specific PE method used in this work - the split-step Fourier method - was selected due to its computational efficiency and conceptual simplicity. However, many other PE methods in common use today may also be applicable to 3D THz tomographic imaging problems. The material presented in this dissertation represents the first step in this direction, laying the foundation for further work in bringing the powerful family of PE methods to bear on $\mathrm{THz}$ phased-array tomographic imaging problems.

\subsection{Executive Summary}

This section outlines the remainder of the dissertation, providing a brief summary of each chapter for quick reference.

Chapter 2 provides an overview of the relevant literature on THz NDE (Sec. 2.1), 3D THz imaging (Sec. 2.2), and PE methods and related physics-based wave propagation approaches (Sec. 2.3). In addition, Sec. 2.4 presents an overview of the literature on analytic methods for modeling the scattering of electromagnetic waves from a layered dielectric sphere relevant to results presented in Appendix A. 
Chapter 3 lays the theoretical framework for the topics combined in this work:

Sec. 3.1 provides a rigorous derivation and analysis of reflection-mode diffraction tomography, of which $\mathrm{THz}$ synthetic aperture tomography is a special case.

Sec. 3.2 derives the PE formulations used throughout the rest of the dissertation.

Sec. 3.2.1 introduces and discusses Fourier back-propagation (FBP) and presents an intuitive geometric approach to determining imaging resolution in a generalized broadband synthetic aperture imaging system.

Sec. 3.2.2 introduces the split-step Fourier (SSF) back-propagation method and presents a rigorous analysis of the error introduced by approximations made in its derivation.

Chapter 4 presents details on the implementation of the methods introduced in Chapter 3 using the THz imaging system at the Northwest Electromagnetics and Acoustics Research Laboratory (NEAR-Lab).

Sec. 4.1 describes the Picometrix T-Ray 4000 system and presents details on its use in implementing the methods described in Chapter 3. Sec. 4.1.1 discusses methods of preprocessing of $\mathrm{THz}$ waveforms to suppress noise and improve temporal/range resolution.

Sec. 4.2 presents results from three experiments conducted using the Picometrix T-Ray 4000 described in the previous section.

Sec. 4.2.1 describes the first experiment, carried out as a "proof of concept," on an over the counter pharmaceutical gelcap. As the constituent materials of the gelcap are unknown, two further experiments 
were conducted on well-characterized test samples to demonstrate and quantify the accuracy of the images produced using SSF backpropagation.

Sec. 4.2.2 describes an experiment involving a well-characterized cylindricallycurved high-density polyethylene sample with high-contrast copper wires embedded within. Results from this experiment were presented at the 2013 SPIE Optics and Photonics conference in San Diego, CA [39].

Sec. 4.2.3 describes an experiment conducted on a specially-designed 3Dprinted test object. Results from this experiment were presented at the 2015 International Radar Symposium (IRS) in Dresden, Germany [40] and are included in a manuscript recently submitted for publication in the IEEE Transactions on Terahertz Science and Technology [41].

Sec. 4.3 relates the analytic expression for the SSF propagator error, presented in Sec. 3.2.2, to the experimental results presented in Sec. 4.2.3. Idealized simulations are then shown to further examine the influence of propagator error on the SSF method's ability to correctly localize the boundaries and estimate the thickness of a dielectric layer in an object under test. These results are featured in a manuscript recently submitted for publication in the IEEE Transactions on THz Science and Technology [41].

Chapter 5 provides closing remarks, suggests avenues for future work in this area, and presents a list of publications that were either authored or co-authored during the course of conducting this research.

Appendix A describes work conducted in the implementation and testing of a Mie series model for the electromagnetic scattering from a layered dielectric sphere. 
This model was originally intended to serve as a source of simulation data for testing the ability of the split-step Fourier method from Sec. 3.2.2 to accurately quantify the thickness of a curved dielectric layer. However, the analysis in Sec. 4.3 proved to be more useful for this purpose. Nonetheless, the work in Mie series scattering from a layered dielectric sphere represents a significant body of the work completed as part of this research and is therefore included in hopes that it may assist in future work in this area. 


\section{Chapter 2}

\section{Literature Review}

As mentioned in Sec. 1.1, this work represents a novel combination of THz technology, phased array imaging, reflection-mode diffraction tomography, and parabolic equation (PE) methods. This section provides context for the contributions made in this dissertation by summarizing the current state of research in these relevant fields. It begins with an overview of $\mathrm{THz}$ NDE and its applications, followed by a discussion of $3 \mathrm{D} \mathrm{THz}$ imaging methods and a summary of relevant work in wave propagation methods used in ultrasound NDE, medical imaging, underwater acoustics, and atmospheric propagation of electromagnetic waves. Finally, an overview of the state of the art in analytic modeling of electromagnetic scattering from a layered dielectric sphere is provided.

\subsection{Terahertz Non-Destructive Evaluation}

Nondestructive evaluation is a very broad area of study for which a wide variety of technologies have been developed. In NDE, either electromagnetic or ultrasonic waves are passed through an object of interest in order to probe its structure with- 
out permanently altering it. In the case of $\mathrm{THz} \mathrm{NDE}$, the objects of interest are typically composed non-polar dielectric materials, which are transparent to $\mathrm{THz}$ radiation. This, combined with the relatively small wavelengths $(0.1-3 \mathrm{~mm})$ and broad bandwidth of pulsed $\mathrm{THz}$ systems allow high resolution imaging of the objects under test.

The earliest results demonstrating the potential of THz imaging for NDE applications were published in the mid to late 1990s, shortly after the development sufficiently powerful THz sources and sensitive $\mathrm{THz}$ receivers. Electronics components, such as packaged semiconductor integrated circuits and floppy disks along with leaf specimens with varying moisture content were among the first objects to be imaged with $\mathrm{THz}$ radiation $[17,18,42]$. These two cases spawned interest in both biomedical and NDE applications of $\mathrm{THz}$ imaging: The ability of $\mathrm{THz}$ imaging to visualize spatially-varying differences in moisture content along with its non-ionizing energy levels spurred interest in $\mathrm{THz}$ imaging for biomedical applications [43, 44] while the potential to visualize conductive electrical contacts and other internal structures within dielectric packaging has driven the interest in THz NDE for electronics components [11-13, 45, 46].

In the wake of the 2003 space shuttle Columbia disaster, $\mathrm{THz}$ NDE was investigated as a potential method of detecting cracks, voids, and debond defects (areas of poor adhesion) in the foam insulation sprayed onto the space shuttle's external fuel tank [47-50] as well as the silica tiles that made up the shuttle's heat shield [51-53]. Other applications for $\mathrm{THz}$ NDE in the aerospace industry include inspection of thermal barrier coatings applied to jet turbine blades [16] and aircraft composite parts [14, $15]$.

THz NDE has also been used by the polymer industry for detecting leaks in plastic 
food packaging $[54,55]$, determining the orientation of glass fibers in reinforced plas-

tics [56], and inspecting weld joints in polymer parts [8], among other applications [9, 10]. The automotive industry has investigated using $\mathrm{THz}$ to monitor the drying and deposition of automotive paint $[6,7,20]$. The field of art history and preservation has also been interested in the use of $\mathrm{THz}$ to analyze the materials used by artists [57] and image hidden paint layers on canvas [58], wood [59], and murals [60, 61].

The most pertinent application to this body of work is the NDE of pharmaceutical tablet coatings $[4,5,62,63]$. Initial explorations into this area involved raster scanning a $\mathrm{THz}$ pulsed transceiver (operating in reflection mode) over a small area of a biconvex pharmaceutical tablet's surface [19]. To limit refractive errors in the TPI data, the scan area was restricted to a small patch near the center of the convex curved surface where the surface normal was nearly vertical. Subsequent work utilized a six-axis robotic arm to expand the scan area over the entire surface [1]. Currently, a typical data collection with the robot arm assembly takes approximately 40-60 minutes per pill. Throughput will need to be increased substantially in order for THz NDE of pharmaceutical tablets to become standard practice.

\subsection{D Terahertz Imaging}

Since the first 2D THz images were taken in the 1990s [42], researchers have used various methods to produce 3D $\mathrm{THz}$ images of objects of interest, the first being the aforementioned TPI of a floppy disk by Mittleman et al. in 1997 [18]. While THz imaging can be done with continuous wave (CW) swept frequency systems [51, 64, 65], the broad bandwidth of pulsed time-domain systems allows a high degree of depth resolution, making it preferable for many NDE applications. Much of the work in 3D 
$\mathrm{THz}$ imaging has consisted of adapting many well-established 3D imaging methods from other fields such as medical imaging, radar, and ultrasound to the $\mathrm{THz}$ band. This can be done with both transmission and reflection mode data.

Among the prime examples of transmission mode 3D THz imaging is the family of methods that borrow from X-ray computed tomography (CT). These methods involve taking transmission measurements, or projections, through the target at varying azimuthal angles. $\mathrm{THz} \mathrm{CT}$ was first demonstrated by Ferguson et al. in 2002 [66, 67], who employed conventional X-ray CT techniques and filtered back projection to generate images of a plastic tube, a sheet of polyethylene twisted into an s-shape, and a turkey bone. Diffraction tomography (DT) - a more generalized version of $\mathrm{CT}$ applicable to objects with features closer in scale to $\mathrm{THz}$ wavelengths - was soon demonstrated by the same group [68] along with THz tomography using both Fresnel zone plate lenses and CCD cameras in a digital holography configuration [69, 70]. Transmission mode THz tomography has also been demonstrated using a parallel plate waveguide as the imaging aperture [71], a quantum cascade laser source [72], an all-electronic THz-CT system operating in the 230-320 GHz band [73], and a fixed-phase-shift method employing a phase-unwrapping algorithm [74]. In addition, the efficiency of generating $\mathrm{THz}$ tomographic images has been increased by using alternative tomographic image reconstruction methods such as tomosynthesis, which allows image reconstruction from a much smaller number of projections than conventional CT [75] as well as wavelet-based de-noising methods that allow faster data acquisition and enhance tomographic images taken with pulsed THz sources [76, 77].

Although initially a promising approach, the pace of development in transmission mode $\mathrm{THz} \mathrm{CD} / \mathrm{DT}$ methods has slowed due to Fresnel losses and refractive effects 
introduced by objects with moderate refractive indices $(n \sim 1.5$ or greater) [78]. Though progress is being made in accounting for these effects [78, 79], they remain among the primary technical obstacles to the mainstreaming of 3D transmission mode imaging of THz-transparent objects.

For thick, lossy, or strongly refracting objects, or cases in which the features of interest are close to the object's outer surface, a reflection mode configuration is more practical for 3D THz imaging. Though some work has been done adapting CT methods to data collected in reflection mode [80], the bulk of 3D THz imaging in reflection mode borrows heavily from the TPI methods pioneered by Mittleman et al. [18], which operate in a similar manner to pulse-echo mode ultrasound. The delay times of the returned $\mathrm{THz}$ echo pulses are mapped to a physical distance using a known or assumed refractive index. The broad bandwidth of pulsed $\mathrm{THz}$ sources leads to a high degree of range (depth) resolution while lateral resolution is determined by $\mathrm{THz}$ optics, such as off-axis parabolic mirrors or focusing lenses. In a typical TPI system, THz pulses from a single monostatic transceiver raster scanned over an object are used to generate a 3D image [18, 20, 21, 62, 81]. However, as with any optical system, the focusing of the $\mathrm{THz}$ beam limits the depth of focus. To image accurately over a greater range, the focal spot size must either be widened, which limits lateral resolution, or the transceiver must be raster scanned in three dimensions.

Phased array methods offer a promising alternative to conventional TPI. In the most general sense of the term, phased-array methods encompass a wide variety of techniques from a diverse range of fields, such as beamforming in underwater acoustics $[82,83]$, synthetic aperture radar $(\mathrm{SAR})$ in microwave remote sensing [84], Fourier holography in optics [85], and phase migration in seismology [86]. Among the earliest 
examples of phased array imaging methods applied to pulsed $\mathrm{THz}$ data was the use of the Huygens-Fresnel principle to back-propagate $\mathrm{THz}$ data collected in transmission mode [87, 88]. Similarly, the use of Kirchhoff migration [89] to generate images from data collected from a single fixed transmitter position and multiple receiver positions and a quasi-optical configuration similar to that employed by optical telescope arrays [90] are among the first demonstrations of reflection-mode $\mathrm{THz}$ phased array imaging. More recently, a synthetic aperture method was developed that employs a focal lens to effect a point-like virtual transceiver which, when raster scanned in two dimensions, can be used to synthesize a 2D planar synthetic array. Delay-and-sum beamforming methods were then used to generate images from the resulting data [91, 92]. Our own research group has also published results from a similar method - THz synthetic aperture (SA) tomography - which applies a set of geometrically-derived frequency domain phase shifts over a broad frequency band to focus the 2D synthetic array to a given parallel plane below the array $[23,24]$. Focusing to a number of such focal planes allows the generation of 3D surface profiles of metallic objects as well as 3D tomographic maps of the interior features of THz-penetrable dielectric objects. While these early results demonstrate the potential of THz SA tomography, a number of simplifying assumptions were made in the processing that limit its applicability to objects with planar boundaries that lie parallel to the array plane. A THz SA focusing method that can accommodate arbitrary, non-planar boundaries (such as curved surfaces) has yet to be developed, and is the primary contribution of this dissertation. 


\subsection{Wave Propagation Methods}

As mentioned previously, TPI borrows heavily from concepts in pulse-echo ultrasound, which is commonplace in NDE and medical applications. Similarly, the use of a focal lens to effect a point-like virtual transceiver for use in synthetic aperture imaging was first pioneered by researchers in ultrasound NDE [93-95] before being adapted for use in $\mathrm{THz}$ imaging systems [23, 24, 91, 92]. Synthetic aperture focusing techniques (SAFTs) are a common form of generating images from synthetic aperture data in both ultrasound and microwave/mm-wave NDE [96-99]. The processing can be accomplished either in the time domain [94, 95, 97, 100] or frequency domain $[96,101,102]$. In the simplest case, SAFT processing assumes signals collected correspond to waves propagating through a homogeneous medium with constant phase velocity throughout the image space $[92,94,95,102]$. Of more practical concern is the case of an object whose refractive index is dissimilar from from the surrounding medium. One simple case of such a situation would be an object with one or more planar boundaries parallel to the aperture plane. This case can be handled using a variety of methods, such as using Snell's law to geometrically derive the time delays [100] or frequency-domain phase shifts $[23,24]$ required to focus the phased-array data to a point of interest, or by using Stolt transforms in an "Omega-K" algorithm [103]. However, more complicated methods must be used for objects with non-planar boundaries, which present laterally-varying refractive index variations.

For objects or environments with both axially and laterally-varying wave speeds, numerical wave-equation based methods are often employed to either model the forwardpropagation of an excitation or back-propagate measured fields back to their points

of origin within an object or environment. One such class of techniques is based on a 
parabolic approximation of the Helmholtz equation. These parabolic equation (PE) methods have been applied to a broad range of science and engineering disciplines [32, 104-106] and may be solved using a variety of methods. Common approaches include finite difference (FD) and finite element (FE) methods [32, 107, 108] and fast Fourier transform (FFT) based methods [29, 31, 32, 109]. Historically, the two methods presented a tradeoff between accuracy (FD/FE) and efficiency (FD), however the development of the split-step Padé method in the late 1990s provides a compromise between the two [110-112]. While PE methods are typically applied to solve 2D propagation problems in underwater acoustics, recent work has been conducted implementing 3D PE solutions using FFT and Padé methods [113-116].

One efficient FFT-based method, namely the split-step Fourier (SSF), is among the most utilized approaches to PE methods, having been applied in medical ultrasound imaging [37, 38], underwater acoustics [31, 32, 114, 116], seismic migration [29], ground-penetrating radar imaging [35, 36], electromagnetic wave propagation in the atmosphere [33, 104], and light propagation in optical fibers [109]. The novel adaptation of the SSF method to $\mathrm{THz}$ synthetic aperture imaging is one of the primary contributions of this work [39-41].

\subsection{Mie Series Solution for Layered Sphere Scattering}

A large part of the work carried out as part of this research was the development and MatLaB implementation of an efficient, stable recursive method for calculating the scattered fields from a layered dielectric sphere - a canonical object presenting a multiply-layered, curved surface. This code was originally intended for use in generating simulation data for quantitative performance evaluation of the physics-based 
synthetic aperture focusing method presented in this work. However, another line of analysis, summarized in Sec. 4.3 eventually proved to be more useful for this purpose. The results of the work on the Mie series scattering model are presented in Appendix A.

Although the early work on the problem of electromagnetic scattering from a sphere was carried out independently by Mie, Debye, and Lorentz [117], it was Mie's name that ended up being attached to the theory. Much of the work on the Mie series solution is summarized by Bohren and Huffman [117]. Though the underlying theory is the same, much of the subsequent work differs in implementation and assumptions about material composition. The most common ("textbook") case of interest assumes plane wave excitation and a perfectly conducting sphere [118]. More general cases of dielectric spheres and coated dielectric spheres are also common [117, 119, 120], however most such resources focus on calculating scattering efficiencies and cross sections as opposed to internal and external complex field amplitude calculations. The complex field amplitudes include the phase of the scattered fields, which is essential for use in generating simulation data suitable for performance evaluation of the physicsbased synthetic aperture focusing method.

There are many numerical approaches used to calculate the Mie series coefficients that satisfy the electromagnetic boundary conditions in a layered dielectric sphere. One of the simplest conceptually utilizes T-matrices [121-123]. However, due to advantages in computational efficiency, recursive methods are often used over T-matrix methods [117, 124-127]. Another consideration is numerical stability of the numerous spherical Bessel function calculations used in calculating the spherical harmonics in the Mie series representation of the scattered and internal field amplitudes. While 
MATLAB software (primarily used in this work) includes a standard package for calculating Bessel functions, the implementations are computationally expensive and may be prohibitively time consuming if many Bessel function calculations need to be carried out. Luckily, alternative methods based on recursive algorithms and continued fractions have been reported on extensively in the literature [128-130]. Another advantage of the published recursive methods [117, 124-127] is their utilization of spherical Bessel function ratio and logarithmic derivative calculations, which are generally more efficient and numerically stable than calculating the functions themselves $[128,130]$. 


\section{Chapter 3}

\section{Theoretical Background}

This chapter lays the theoretical framework for the contributions made in this dissertation. As $\mathrm{THz}$ synthetic aperture tomography can be considered a special case of reflection mode diffraction tomography, the section begins with a theoretical treatment of the latter. Parabolic equation methods are then introduced along with the derivation of two special cases - Fourier back-propagation and split-step Fourier backpropagation - that form the basis of the image processing methods introduced in this dissertation. A simple geometrical approach to determining imaging resolution in a generalized broadband synthetic aperture imaging system is presented as well as an analysis of the error introduced by approximations made in the derivation of the split-step Fourier method.

\subsection{Reflection Mode Diffraction Tomography}

The word "tomography" is derived from the Greek word tomos, which means slice,

and the suffix -graphy, which generally refers to the process of generating an image (i.e. photography, sonography, holography, etc.). The term therefore refers to the 
process of generating a 3D image of an object under test from a stack of 2D image slices. Typically in the context of 3D THz imaging, the term "tomography" is used to describe the generation of a 3D image using TPI data or by adapting methods from X-Ray computed tomography (CT) [22], in which the tomographic slices are reconstructed from data collected from a sensor rotating around the object (or person) under test (i.e. $[70,80])$.

The term "diffraction tomography" as used in this section refers to a generalized inverse scattering problem originally treated by Emil Wolf in 1969-70 [131, 132]. One special case of diffraction tomography, often referred to as the synthetic aperture focusing technique (SAFT) in the ultrasound NDE community [96, 97, 101, 133, 134], operates in reflection mode using a single monostatic transceiver element with a diverging beam. A specific implementation of ultrasound SAFT that utilizes the diverging beam below the focal point of a focused ultrasonic transceiver [95] forms the basis of subsequent work in THz SA tomography [23, 24, 91, 92]. As such, the THz SA tomography method presented in this and previous works can be considered a special case of reflection mode diffraction tomography.

The collection geometry for this method is illustrated in Fig. 3.1.1. An isotropic point source lies in the $x y$ plane at $\mathbf{r}_{0}=\left(x, y, z_{0}\right)$ where it illuminates an object with broadband $\mathrm{THz}$ pulses whose complex spectrum $S(\omega)$ is a function of the temporal angular frequency $\omega$ in radians/second, where time dependence is assumed to be given by $e^{-i \omega t}$. The object under test is represented by a localized 3D region of refractive index $n(\mathbf{r})>1$ in an otherwise free space medium with $n(\mathbf{r})=1$, where $\mathbf{r}=\left(x, y, z>z_{0}\right)$ is the $3 \mathrm{D}$ position vector in the region below the array plane. While in general, the refractive index $n(\mathbf{r})$ may also be dependent on the temporal frequency 


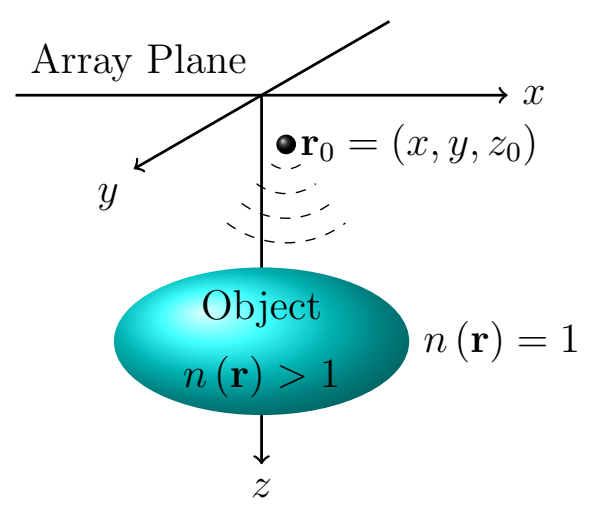

Figure 3.1.1: Imaging geometry assumed in THz SA tomography formulation. The object under test, represented by a closed region of refractive index $n(\mathbf{r})>1$, is illuminated by an isotropic point transceiver at $\mathbf{r}_{0}=\left(x, y, z_{0}\right)$ in the $x y$ plane.

$\omega$, it is assumed here to be frequency independent for simplicity of notation.

From scalar diffraction theory $[85,131]$, the complex field amplitudes $\psi(\mathbf{r}, \omega)$ in the region are treated as solutions to the inhomogeneous Helmholtz equation,

$$
\left[\nabla^{2}+n^{2}(\mathbf{r}) k^{2}\right] \psi(\mathbf{r}, \omega)=-S(\omega) \delta\left(\mathbf{r}-\mathbf{r}_{0}\right),
$$

where the term on the right hand side represents the isotropic point transceiver source at $\mathbf{r}_{0}$. By defining the scattering potential,

$$
f(\mathbf{r}) \equiv n^{2}(\mathbf{r})-1,
$$

the inhomogeneous Helmholtz equation (3.1.1) can be rewritten as

$$
\left[\nabla^{2}+k^{2}\right] \psi(\mathbf{r}, \omega)=-S(\omega) \delta\left(\mathbf{r}-\mathbf{r}_{0}\right)-k^{2} f(\mathbf{r}) \psi(\mathbf{r}, \omega),
$$

where the second term on the right-hand side takes the form of an additional equivalent source that accounts for all the secondary scattering sources introduced by the 
object. The solution to (3.1.3) can be expressed as a sum of incident and scattered components, respectively, as

$$
\psi(\mathbf{r}, \omega)=\psi_{i}(\mathbf{r}, \omega)+\psi_{s}(\mathbf{r}, \omega)
$$

where

$$
\begin{aligned}
& \psi_{i}(\mathbf{r}, \omega)=S(\omega) g\left(\mathbf{r}_{0}-\mathbf{r}, \mathbf{k}\right) \\
& \psi_{s}(\mathbf{r}, \omega)=k^{2} \iint_{-\infty}^{\infty} f\left(\mathbf{r}^{\prime}\right) \psi\left(\mathbf{r}^{\prime}, \omega\right) g\left(\mathbf{r}-\mathbf{r}^{\prime}, \mathbf{k}\right) d^{3} \mathbf{r}^{\prime}
\end{aligned}
$$

and the 3D scalar Green's function is given by

$$
g(\mathbf{r}, \mathbf{k})=\frac{e^{i \mathbf{k} \cdot \mathbf{r}}}{4 \pi|\mathbf{r}|}
$$

in terms of the position vector $\mathbf{r}$ and the wave vector $\mathbf{k}=\left(k_{x}, k_{y}, k_{z}\right)$, whose magnitude is given by the wavenumber $k=\omega / c=|\mathbf{k}|$. Under the Born approximation [96], weak scattering is assumed such that $\left|\psi_{i}(\mathbf{r})\right|>>\left|\psi_{s}(\mathbf{r})\right|$, which linearizes (3.1.6), allowing it to be rewritten in terms of (3.1.5) as

$$
\begin{aligned}
\psi_{s}(\mathbf{r}, \omega) & \approx k^{2} \iint_{-\infty}^{\infty} f\left(\mathbf{r}^{\prime}\right) \psi_{i}\left(\mathbf{r}^{\prime}, \omega\right) g\left(\mathbf{r}-\mathbf{r}^{\prime}, \mathbf{k}\right) d^{3} \mathbf{r}^{\prime} \\
& =k^{2} S(\omega) \iiint_{-\infty}^{\infty} f\left(\mathbf{r}^{\prime}\right) g\left(\mathbf{r}_{0}-\mathbf{r}^{\prime}, \mathbf{k}\right) g\left(\mathbf{r}-\mathbf{r}^{\prime}, \mathbf{k}\right) d^{3} \mathbf{r}^{\prime}
\end{aligned}
$$

For the case of monostatic backscatter, the source and receiver are collocated and the 
scattered fields detected by the receiver are given by

$$
\psi_{s}\left(\mathbf{r}_{0}, \omega\right)=k^{2} S(\omega) \iint_{-\infty}^{\infty} f\left(\mathbf{r}^{\prime}\right) g^{2}\left(\mathbf{r}_{0}-\mathbf{r}^{\prime}, \mathbf{k}\right) d^{3} \mathbf{r}^{\prime}
$$

where the squared Green's function in the integrand can be expressed as

$$
\begin{aligned}
g^{2}\left(\mathbf{r}_{0}-\mathbf{r}^{\prime}, \mathbf{k}\right) & =\frac{e^{i 2 k\left|\mathbf{r}_{0}-\mathbf{r}^{\prime}\right|}}{\left(4 \pi\left|\mathbf{r}_{0}-\mathbf{r}^{\prime}\right|\right)^{2}} \\
& =\frac{g\left(\mathbf{r}_{0}-\mathbf{r}^{\prime}, 2 \mathbf{k}\right)}{4 \pi\left|\mathbf{r}_{0}-\mathbf{r}^{\prime}\right|}
\end{aligned}
$$

From (3.1.10), it can be seen that monostatic backscatter measurements introduce an effective doubling of the phase term in the Green's function due to the round-trip travel of each scattered pulse from the transceiver to the scatterer and back. In the time domain, this phase shift is equivalent to a time delay $t_{0}$, i.e.

$$
\omega t_{0}=2 k\left|\mathbf{r}_{0}-\mathbf{r}^{\prime}\right|
$$

Solving (3.1.11) for the range $\left|\mathbf{r}_{0}-\mathbf{r}^{\prime}\right|$ yields

$$
\left|\mathbf{r}_{0}-\mathbf{r}^{\prime}\right|=\frac{c}{2} t_{0}
$$

Thus, this doubling of the wavenumber is equivalent of the halving of the phase velocity typically used to determine the range to the scatterer in a typical monostatic measurement (such as in TPI, radar, active sonar, etc.). The round-trip travel also introduces an additional factor of $4 \pi\left|\mathbf{r}^{\prime}-\mathbf{r}_{0}\right|$ in the denominator due to the additional spherical spreading of the wavefront. Dividing both sides of (3.1.9) by $k^{2} S(\omega)$ and 
substituting (3.1.10a) yields

$$
\frac{\psi_{s}\left(\mathbf{r}_{0}, \omega\right)}{k^{2} S(\omega)}=\iint_{-\infty}^{\infty} \int f\left(\mathbf{r}^{\prime}\right) \frac{e^{i 2 k\left|\mathbf{r}_{0}-\mathbf{r}^{\prime}\right|}}{\left(4 \pi\left|\mathbf{r}_{0}-\mathbf{r}^{\prime}\right|\right)^{2}} d^{3} \mathbf{r}
$$

In practice, the division by $S(\omega)$ amounts to deconvolution with the source spectrum - often a Wiener deconvolution for noisy band-limited signals [135], the process of which will be described in Sec. 4.1.1. Taking the derivative of (3.1.13) with respect to $k$ yields

$$
\begin{aligned}
\frac{\partial}{\partial k}\left[\frac{\psi_{s}\left(\mathbf{r}_{0}, \omega\right)}{k^{2} S(\omega)}\right] & =\iint_{-\infty}^{\infty} f\left(\mathbf{r}^{\prime}\right) \frac{\partial}{\partial k} \frac{e^{i 2 k\left|\mathbf{r}_{0}-\mathbf{r}^{\prime}\right|}}{\left(4 \pi\left|\mathbf{r}_{0}-\mathbf{r}^{\prime}\right|\right)^{2}} d^{3} \mathbf{r} \\
& =\frac{i}{2 \pi} \iint_{-\infty}^{\infty} f\left(\mathbf{r}^{\prime}\right) \frac{e^{i 2 k\left|\mathbf{r}_{0}-\mathbf{r}^{\prime}\right|}}{4 \pi\left|\mathbf{r}_{0}-\mathbf{r}^{\prime}\right|} d^{3} \mathbf{r}
\end{aligned}
$$

By the differentiation property of the Fourier transform, this differentiation in $k$ (applied in the frequency domain) becomes a range-dependent amplification (i.e. multiplication by $c t / 2$ in the time domain) that compensates for the $\left|\mathbf{r}_{0}-\mathbf{r}^{\prime}\right|$ in the denominator of (3.1.10) [135]. After scaling both sides of (3.1.14) by $-2 \pi i$, the resulting preprocessed signals are given by

$$
\begin{aligned}
\psi_{p}\left(\mathbf{r}_{0}, \omega\right) & \equiv-2 \pi i \frac{\partial}{\partial k}\left[\frac{\psi_{s}\left(\mathbf{r}_{0}, \omega\right)}{k^{2} S(\omega)}\right] \\
& =\iiint_{-\infty}^{\infty} f\left(\mathbf{r}^{\prime}\right) g\left(\mathbf{r}_{0}-\mathbf{r}^{\prime}, 2 \mathbf{k}\right) d^{3} \mathbf{r}^{\prime}
\end{aligned}
$$

From (3.1.15), the fields in the aperture plane (under the Born approximation) are the superposition of the fields emanating from a distribution of isotropic Huygens' point 
reflectors (Green's functions with wavenumber $2 k$ ) below the array, each weighted by the scattering potential $f(\mathbf{r})$, which falls to 0 outside the object. Equivalently, (3.1.15) can be seen to be a 3-dimensional spatial convolution of the scattering potential in (3.1.2) with the monostatic Green's function (whose wavenumber is $2 k$ ).

At this point in the derivation, it's helpful to introduce the 2D spatial Fourier transform,

$$
\begin{aligned}
\tilde{h}\left(k_{x}, k_{y}, z\right) & =\mathcal{F}_{x, y}\{h(x, y, z)\} \\
& =\int_{-\infty}^{\infty} h(x, y, z) e^{-i\left(k_{x} x+k_{y} y\right)} d x d y
\end{aligned}
$$

and the corresponding 2D inverse Fourier transform,

$$
\begin{aligned}
h(x, y, z) & =\mathcal{F}_{k_{x}, k_{y}}^{-1}\left\{\tilde{h}\left(k_{x}, k_{y}, z\right)\right\} \\
& =\frac{1}{(2 \pi)^{2}} \int_{-\infty}^{\infty} \tilde{h}\left(k_{x}, k_{y}, z\right) e^{i\left(k_{x} x+k_{y} y\right)} d k_{x} d k_{y},
\end{aligned}
$$

where $k_{x}=2 k \sin \theta \cos \phi$ and $k_{y}=2 k \sin \theta \sin \phi$ are the Fourier spatial frequencies in the $x$ and $y$ directions, which correspond to plane-wave components (represented by the complex exponential Fourier kernel) propagating in the direction specified in spherical coordinates by the zenithal angle $\theta$ from the $z$ axis and the azimuthal angle $\phi$ from the $x$ axis as shown in Fig. 3.1.2. The inverse Fourier transform (3.1.17) therefore represents the function $h(x, y, z)$ as a superposition of plane waves, each with complex amplitude $\tilde{h}\left(k_{x}, k_{y}, z\right)$. Conversely, the Fourier transform (3.1.16) decomposes the function $h(x, y, z)$ into the angular spectrum of plane waves $\tilde{h}\left(k_{x}, k_{y}, z\right)$. 


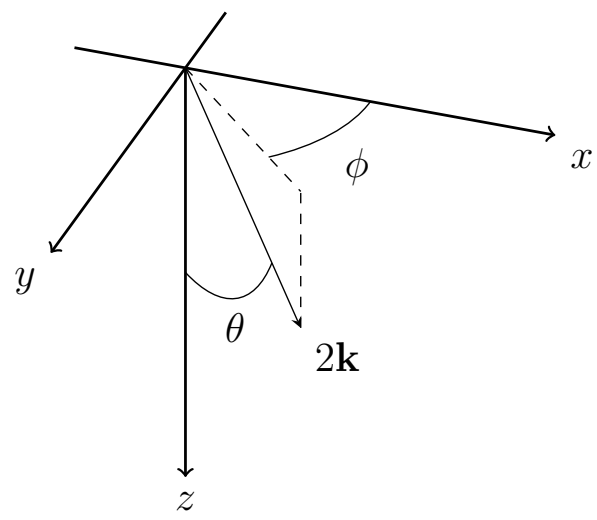

Figure 3.1.2: Wave vector shown in terms of spherical coordinates.

By decomposing $\psi_{p}\left(\mathbf{r}_{0}, \omega\right)$ into its angular plane wave spectrum via (3.1.16), the 3D convolution integral in $(3.1 .15)$ can be reduced to a $1 \mathrm{D}$ convolution in $z$,

$$
\tilde{\psi}_{p}\left(k_{x}, k_{y}, z_{0}, \omega\right)=\tilde{f}\left(k_{x}, k_{y}, z\right) * \mathcal{F}_{x, y}\{g(\mathbf{r}, 2 \mathbf{k})\}
$$

where

$$
\tilde{f}\left(k_{x}, k_{y}, z\right)=\mathcal{F}_{x, y}\{f(x, y, z)\}
$$

is the 2D Fourier transform of the scattering potential. The 2D Fourier transform of the Green's function can be obtained from the Weyl identity [136],

$$
\begin{aligned}
\frac{e^{i 2 k r}}{4 \pi r} & =\frac{i}{8 \pi^{2}} \int_{-\infty}^{\infty} \frac{e^{i k_{x} x+i k_{y} y+i \gamma|z|}}{\gamma} d k_{x} d k_{y} \\
& =\frac{1}{(2 \pi)^{2}} \iint_{-\infty}^{\infty} \frac{i}{2} \frac{e^{i \gamma|z|}}{\gamma} e^{i k_{x} x+i k_{y} y} d k_{x} d k_{y} \\
& =\mathcal{F}_{k_{x}, k_{y}}^{-1}\left\{\frac{i}{2} \frac{e^{i \gamma|z|}}{\gamma}\right\}
\end{aligned}
$$


where $r=\sqrt{x^{2}+y^{2}+z^{2}}$ and $[96,137]$

$$
\gamma=k_{z}\left(k, k_{x}, k_{y}\right)=\sqrt{(2 k)^{2}-k_{x}^{2}-k_{y}^{2}}
$$

Suppressing the functional dependence of $k_{z}$ in (3.1.21) for simplicity of notation, the convolution in (3.1.18) can be rewritten as

$$
\tilde{\psi}_{p}\left(k_{x}, k_{y}, z_{0}, \omega\right)=\frac{i}{2} \int_{-\infty}^{\infty} \tilde{f}\left(k_{x}, k_{y}, z^{\prime}\right) \frac{e^{i k_{z}\left|z_{0}-z^{\prime}\right|}}{k_{z}} d z^{\prime}
$$

Since the array plane lies above the object as shown in Fig. 3.1.1, $z^{\prime}>z_{0}$ and $\left|z_{0}-z^{\prime}\right|=z^{\prime}-z_{0}$ in the complex exponential of (3.1.22), which can then be rewritten as

$$
\tilde{\psi}_{p}\left(k_{x}, k_{y}, z_{0}, \omega\right)=\frac{i}{2 k_{z}} e^{-i k_{z} z_{0}} \int_{-\infty}^{\infty} \tilde{f}\left(k_{x}, k_{y}, z^{\prime}\right) e^{i k_{z} z^{\prime}} d z^{\prime}
$$

The remaining integral in (3.1.23) can be seen to be in the form of a Fourier transform in $z$, which by invoking the Fourier conjugation property yields

$$
\begin{aligned}
\int_{-\infty}^{\infty} \tilde{f}\left(k_{x}, k_{y}, z^{\prime}\right) e^{i k_{z} z^{\prime}} d z^{\prime} & =\left[\int_{-\infty}^{\infty} \tilde{f}^{*}\left(k_{x}, k_{y}, z^{\prime}\right) e^{-i k_{z} z^{\prime}} d z^{\prime}\right]^{*} \\
& =\left[\tilde{f}^{*}\left(k_{x}, k_{y},-k_{z}\right)\right]^{*} \\
& =\tilde{f}\left(k_{x}, k_{y},-k_{z}\right)
\end{aligned}
$$

where the superscript “*” represents complex conjugation. Substituting (3.1.24) back 
into (3.1.23) yields

$$
\tilde{\psi}_{p}\left(k_{x}, k_{y}, z_{0}, \omega\right)=\frac{i}{2 k_{z}} e^{-i k_{z} z_{0}} \tilde{f}\left(k_{x}, k_{y},-k_{z}\right),
$$

which relates the 2D Fourier transform of the preprocessed fields in the array plane, $\tilde{\psi}_{p}\left(k_{x}, k_{y}, z_{0}, \omega\right)$, to the $3 \mathrm{D}$ Fourier transform of the scattering potential $\tilde{f}\left(k_{x}, k_{y},-k_{z}\right)$, whose $-k_{z}$ dependence is due to the fact that only fields propagating in the $-z$ direction will be received by the array, given the $e^{-i \omega t}$ time convention. Ignoring the decaying evanescent field components for which $(2 k)^{2}<k_{x}^{2}+k_{y}^{2}$ results in

$$
\tilde{\psi}_{p}\left(k_{x}, k_{y}, z_{0}, \omega\right)=\frac{i}{2 k_{z}} e^{-i k_{z} z_{0}} \tilde{f}\left(k_{x}, k_{y},-k_{z}\right) u\left[(2 k)^{2}-k_{x}^{2}-k_{y}^{2}\right],
$$

where $u\left[(2 k)^{2}-k_{x}^{2}-k_{y}^{2}\right]$ is the unit step function.

In this final form, (3.1.26) can be considered a statement of the Fourier slice theorem as it applies to reflection mode diffraction tomography [96, 135]: At each temporal frequency component $\omega$, the $2 \mathrm{D}$ spatial Fourier transform of the data in the array plane samples a hemispherical "slice" of radius $2 k=2 \omega / c$ through the 3D Fourier transform of the scattering potential $\tilde{f}\left(k_{x}, k_{y},-k_{z}\right)$. This so-called Ewald hemisphere [96] is illustrated in Fig. 3.1.3. For broadband data, this information is 'filled in' by sampling over multiple hemispherical slices in the 3D Fourier domain, each with a different radius, corresponding to a different temporal frequency $\omega$. Though in principle it would be possible to construct an image of the scattering potential by applying an inverse 3D Fourier transform of the data in (3.1.26), it is much more practical to carry out the image reconstruction by applying back-propagation methods such as those to be described in the following section [135, 137]. It should also be noted that the $2 k$ radius in the Fourier domain corresponds directly to the twofold 


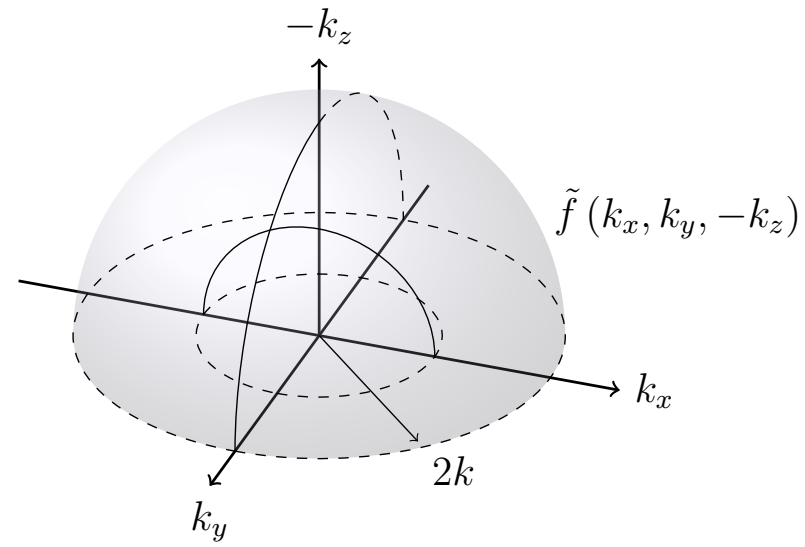

Figure 3.1.3: Illustration of the Ewald hemisphere given by the support of (3.1.26) over which the 3D Fourier transform of the scattering potential is sampled.

improvement in imaging resolution (for a given aperture size) often associated with synthetic aperture imaging $[137,138]$. This improvement comes at a price however, as the spatial sampling frequency must also be doubled, reducing the maximum array spacing from $\lambda / 2$ to $\lambda / 4[137,138]$.

\subsection{Parabolic Equation Methods}

Having collected the data in the array plane as described in Sec. 3.1, the attention turns to converting the data into information about the structure of the object as represented by its scattering potential (3.1.2). This is accomplished by numerically propagating the fields received coherently in the array plane shown in Fig. 3.1.1 back to their points of origin within the object. This is easily accomplished using the exploding reflector model commonly used in seismology [29], in which the object is assumed to scatter the $\mathrm{THz}$ pulses as if it were composed of a distribution of isotropic Huygens point reflectors that scatter the fields back to their points of origin in the array plane. The scattered fields, sampled in the array plane and preprocessed as 
described in Sec. 3.1, are treated as solutions to the scalar Helmholtz equation,

$$
\left[\nabla^{2}+\frac{\omega^{2}}{v_{p}^{2}(\mathbf{r})}\right] \psi(\mathbf{r}, \omega)=0
$$

where the subscript $p$ applied to the preprocessed fields in (3.1.15) has been dropped for simplicity of notation and the phase velocity

$$
v_{p}(\mathbf{r})=\frac{c}{2 n(\mathbf{r})}
$$

depends on the refractive index distribution $n(\mathbf{r})$ within the object space. The factor of 2 in the denominator accounts for the round-trip travel time from each point transceiver to each scatterer and back. Substituting (3.2.2) into (3.2.1) allows rewriting the Helmholtz equation as

$$
\left[\nabla^{2}+(2 k)^{2} n^{2}(\mathbf{r})\right] \psi(\mathbf{r}, \omega)=0
$$

Following the common derivations in the literature [29, 32, 109], the operator

$$
Q(\mathbf{r}) \equiv \sqrt{(2 k)^{2} n^{2}(\mathbf{r})+\frac{\partial^{2}}{\partial x^{2}}+\frac{\partial^{2}}{\partial y^{2}}}
$$

is then defined such that

$$
\left[\frac{\partial}{\partial z}+i Q(\mathbf{r})\right]\left[\frac{\partial}{\partial z}-i Q(\mathbf{r})\right]=\frac{\partial^{2}}{\partial z^{2}}+Q^{2}(\mathbf{r})+i\left[Q \frac{\partial}{\partial z}-\frac{\partial Q}{\partial z}\right]
$$

For weakly $z$-dependent propagation, the cross terms on the right hand side can be neglected [32, 33], resulting in the parabolic form of the homogeneous Helmholtz 
equation,

$$
\left[\frac{\partial}{\partial z}+i Q(\mathbf{r})\right]\left[\frac{\partial}{\partial z}-i Q(\mathbf{r})\right] \psi(\mathbf{r}, \omega)=0
$$

in terms of the operator $Q(\mathbf{r})$ in (3.2.4). This effectively decomposes the $2^{\text {nd }}$-order partial differential equation (Helmholtz equation) into the product of two $1^{\text {st }}$-order partial differential equations, representing two field components propagating in the positive and negative $z$-directions. Given the $e^{-i \omega t}$ time dependence, the fields in the array are related to those in an arbitrary plane $\left(z=z^{\prime}\right)$ in the half space below the array (see Fig. 3.1.1) by

$$
\psi\left(x, y, z^{\prime}, \omega\right)=\psi\left(x, y, z_{0}, \omega\right) \exp \left(i \int_{z_{0}}^{z^{\prime}} Q(x, y, \zeta) d \zeta\right)
$$

The fields $\psi\left(x, y, z_{0}, \omega\right)$ collected in the array plane can therefore be numerically extrapolated backward in time (and space) to the image plane $z^{\prime}$ by applying a complex exponential of the form of that in $(3.2 .7)[29,36]$. In practice, the propagation operator (3.2.4) is approximated in various ways to simplify the integral in $(3.2 .7)[32,34]$. Two such approximations are introduced in the following sections - namely, Fourier back-propagation (FBP), which assumes the fields are back-propagated through free space [85] and therefore does not correct for refraction within the scatterer, and Split-Step Fourier (SSF) back-propagation [29]; an implementation of a wide-angle parabolic equation (PE) method that does account for refraction.

\subsubsection{Fourier Back-Propagation}

In Fourier back-propagation (FBP), the image space is treated as being composed entirely of free space $(n(\mathbf{r})=1)$, which removes the spatial dependence of the prop- 
agation operator (3.2.4) and allows the propagation in (3.2.7) to be easily applied in the spatial frequency domain. Under these conditions, the second derivative terms $\partial^{2} / \partial x^{2}$ and $\partial^{2} / \partial y^{2}$ in (3.2.4) simplify to $-k_{x}^{2}$ and $-k_{y}^{2}$, respectively, resulting in

$$
\begin{aligned}
Q(\mathbf{r}) & \approx \sqrt{(2 k)^{2}-k_{x}^{2}-k_{y}^{2}} \\
& =k_{z}
\end{aligned}
$$

The integral in (3.2.7) then simplifies, turning the complex exponential into a phase shift $e^{i k_{z}\left(z^{\prime}-z_{0}\right)}$ that can easily be applied in the 2D spatial frequency domain [85], i.e.

$$
\psi_{F B P}\left(x, y, z^{\prime}, \omega\right)=\mathcal{F}_{k_{x}, k_{y}}^{-1}\left\{\mathcal{F}_{x, y}\left\{\psi\left(x, y, z_{0}, \omega\right)\right\} e^{i k_{z}\left(z^{\prime}-z_{0}\right)}\right\}
$$

where $\mathcal{F}_{x, y}\{\cdot\}$ and $\mathcal{F}_{k_{x}, k_{y}}\{\cdot\}$ are the forward and inverse Fourier transforms in (3.1.16) and (3.1.17), respectively. After back-propagating the fields to the desired image plane, the image intensity is obtained by integrating over temporal frequency,

$$
I_{F B P}\left(x, y, z^{\prime}\right)=\left|\frac{1}{2 \pi} \int_{-\infty}^{\infty} \psi_{F B P}\left(x, y, z^{\prime}, \omega\right) d \omega\right|^{2}
$$

The process is then repeated for multiple depths $z^{\prime}$ until a full 3D tomographic image is constructed.

Previous work in 3D $\mathrm{THz}$ synthetic aperture imaging [23, 24, 91] has demonstrated focusing methods very similar to FBP to be well-suited to imaging conductive and/or opaque objects, as well as transparent dielectric objects with planar boundaries aligned parallel to the array plane. However, generating accurate 3D tomographic images of transparent objects with non-planar or curved boundaries requires 
correcting for refraction within the object. Given knowledge of the refractive indices of the object's constituent materials (likely to be known a priori in a manufacturing application) and the 3D location of its boundaries (obtainable from FBP-processed 3D tomographic images), the refraction can be quickly and efficiently be corrected for by reprocessing the $\mathrm{THz}$ SA data using the split-step Fourier (SSF) back-propagation method described in Sec. 3.2.2.

\section{Broadband Imaging Resolution: A Geometrical Interpretation}

Typical discussions of imaging resolution assume narrow band illumination from continuous wave sources such as lasers. As a result, the imaging resolution is often given in terms of the optical or acoustic wavelength, $\lambda$. Though useful, this line of analysis is slightly cumbersome and unintuitive for imaging systems that use broad band pulsed sources such as $\mathrm{THz}$ time domain imaging systems. In this case, the signals transmitted are typically time domain waveforms consisting of pulses whose time duration $\tau$, or equivalently, bandwidth $B=1 / \tau$, determines the range resolution according to the classical radar range resolution equation,

$$
\delta r=\frac{c}{2} \tau=\frac{c}{2 B}
$$

For a transceiver at the point $\mathbf{r}_{0}=\left(x, y, z_{0}\right)$ in the array plane in Fig. 3.1.1 and a point scatterer in free space below at $\mathbf{r}^{\prime}=\left(x^{\prime}, y^{\prime}, z^{\prime}\right)$, the reflected echo pulse will arrive at time $t=\frac{2}{c}\left|\mathbf{r}_{0}-\mathbf{r}^{\prime}\right|$. For a single isotropic transceiver, this echo pulse can be thought of as being effectively projected over a sphere of radius $\left|\mathbf{r}_{0}-\mathbf{r}^{\prime}\right|$ centered at the transceiver's location $\mathbf{r}_{0}$. Furthermore, given the range resolution in (3.2.11), the support of this spherically-projected waveform can be confined to the spherical 


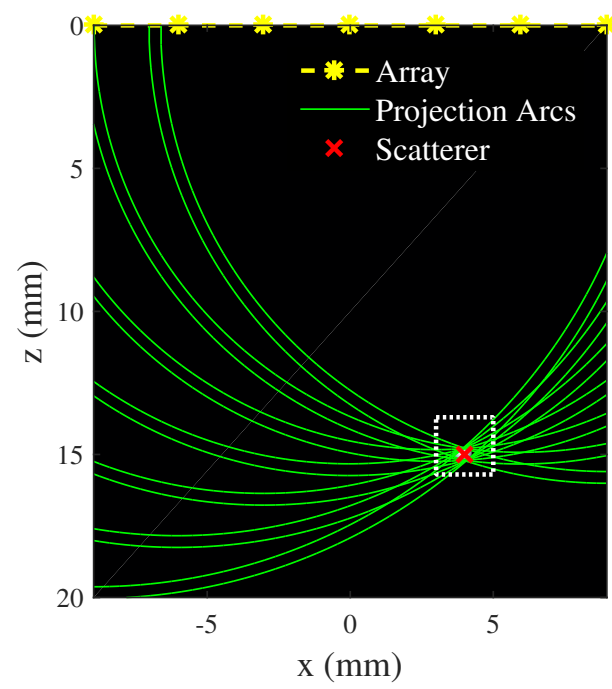

(a)

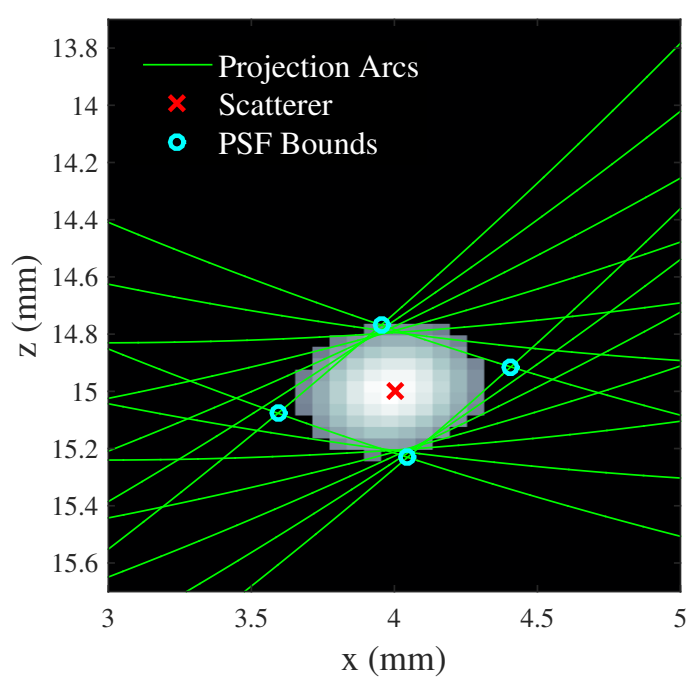

(b)

Figure 3.2.1: Image of a point scatterer at $\left(x^{\prime}, z^{\prime}\right)=(4 \mathrm{~mm}, 15 \mathrm{~mm})$ from 2D simulation data. (a) shows the resulting image along with annular arcs of radial thickness $\delta r=0.41 \mathrm{~mm}$ extending from elements of the array to the location of the scatterer. The rectangle around the scatterer indicates the bounds of the zoomed image (b) which shows the point spread function (PSF) centered at the scatterer's location. The four points bounding the PSF correspond to the intersection points of the annuli from the outermost array elements.

shell with outer radius $\left|\mathbf{r}_{0}-\mathbf{r}^{\prime}\right|+\delta r / 2$ and inner radius $\left|\mathbf{r}_{0}-\mathbf{r}^{\prime}\right|-\delta r / 2$. The process of image reconstruction can then be conceptualized as applying a time delay (or equivalently, a phase shift) to each waveform from each sensor in the array such that their spherical projections overlap and add coherently at the image point [135, 138].

This is illustrated in Fig. 3.2.1 for a set of 2D simulation data in which a 300element linear array was used to image a point scatterer below the array at $\left(x^{\prime}, z^{\prime}\right)=$ (4 mm, $15 \mathrm{~mm})$. The excitation waveforms used in the simulation were Gaussian pulses with $\tau=2.73 \mathrm{ps}$, full-width at half-max (FWHM), which from (3.2.11) corresponds to a range resolution $\delta r=0.41 \mathrm{~mm}$. The backscattered fields on the array of length $L_{x}=17.9 \mathrm{~mm}$ were then calculated and used to form an image via FBP as described above. In the $2 \mathrm{D}$ simulation, the spherical shells indicating the projection bounds 
$\left|\mathbf{r}_{0}-\mathbf{r}^{\prime}\right| \pm \delta r / 2$ become annular arcs centered at the array elements and extending to the point scatterer as shown in Fig. 3.2.1a.

Imaging system performance is typically characterized in terms of a point spread function (PSF), which quantifies the imaging resolution in terms of its impulse response - i.e., the extent to which the image of a point of zero size (such as a delta function) is blurred by the imaging system [85]. Fig. 3.2.1b shows a zoomed in view of the region around the image of the point scatterer, indicated by the rectangle in Fig. 3.2.1a. The image's color map is scaled to omit any pixels whose value is less than half the maximum. Fig. 3.2.1b is therefore an image of the PSF (FWHM) of the SA tomography method for the array configuration and excitation waveform used in the simulation space.

From Fig. 3.2.1b, it can be seen that the PSF is bounded by four points corresponding to the points of intersection of the annuli centered at the outermost array elements. The lateral and axial distances between these four points can therefore be used to quantify the axial and lateral extent of the PSF in terms of the range resolution $\delta r$ of the excitation waveform and the lateral extent $L_{x}$ of the aperture. Specifically, the lateral resolution is given by

$$
x_{\mathrm{res}}=x_{\mathrm{right}}-x_{\mathrm{left}},
$$

where

$$
x_{\mathrm{left}}=\frac{\left(r_{-}+\frac{\delta r}{2}\right)^{2}-\left(r_{-}-\frac{\delta r}{2}\right)^{2}}{2 L_{x}}
$$


and

$$
x_{\text {right }}=\frac{\left(r_{-}-\frac{\delta r}{2}\right)^{2}-\left(r_{-}+\frac{\delta r}{2}\right)^{2}}{2 L_{x}}
$$

are the $x$ coordinates of the points to the left and right side of the scatterer in Fig. 3.2.1 given in terms of

$$
r_{-}=\sqrt{\left(x^{\prime}+\frac{L_{x}}{2}\right)^{2}+z^{\prime 2}}
$$

the radial distance from the leftmost array element to the image point in Fig. 3.2.1, and

$$
r_{+}=\sqrt{\left(x^{\prime}-\frac{L_{x}}{2}\right)^{2}+z^{\prime 2}}
$$

the radial distance from the rightmost array element to the image point. Likewise, the axial resolution is given by

$$
z_{\text {res }}=z_{\text {below }}-z_{\text {above }}
$$

where

$$
z_{\text {below }}=\sqrt{\left(r_{-}+\frac{\delta r}{2}\right)^{2}-\left[\frac{\left(r_{-}+\frac{\delta r}{2}\right)^{2}-\left(r_{+}+\frac{\delta r}{2}\right)^{2}+L_{x}^{2}}{2 L_{x}}\right]^{2}}
$$

and

$$
z_{\text {above }}=\sqrt{\left(r_{-}-\frac{\delta r}{2}\right)^{2}-\left[\frac{\left(r_{-}-\frac{\delta r}{2}\right)^{2}-\left(r_{+}-\frac{\delta r}{2}\right)^{2}+L_{x}^{2}}{2 L_{x}}\right]^{2}}
$$




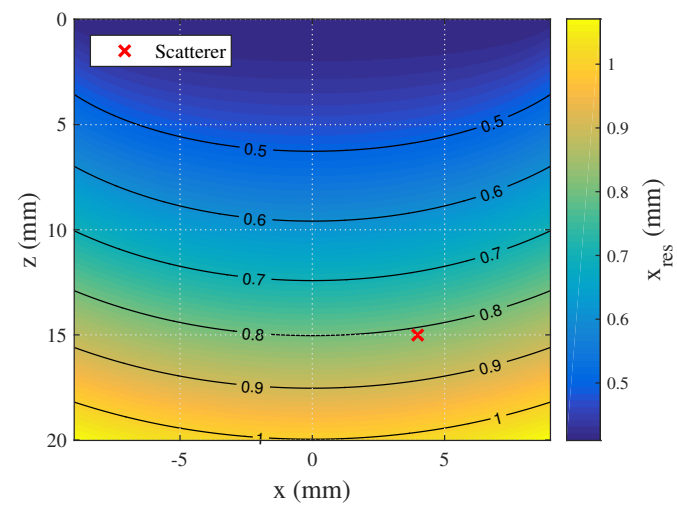

(a)

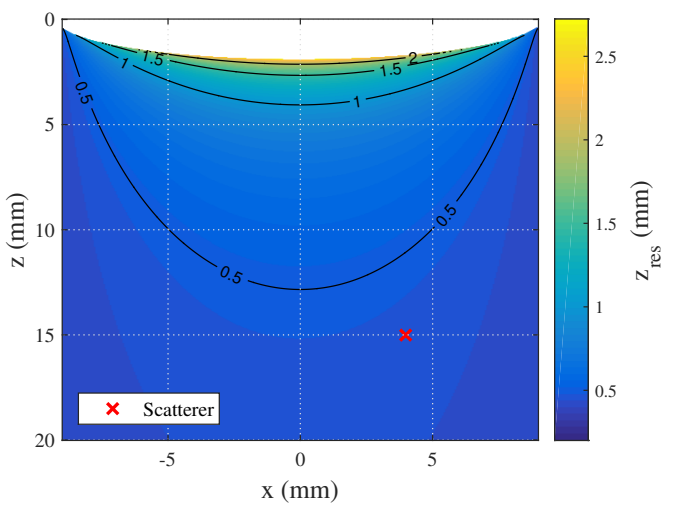

(b)

Figure 3.2.2: Axial (a) and lateral (b) resolution calculated for the simulation space in Fig. 3.2.1 using expressions (3.2.12) and (3.2.17). The red " $\times$ " indicates the location of the scatterer in Fig. 3.2.1 in the parameter space.

are the $z$ coordinates of the points above and below the scatterer in Fig. 3.2.1.

As the expressions indicate, the resolution varies spatially as shown in Fig. 3.2.2 for the $2 \mathrm{D}$ simulation data shown in Fig. 3.2.1. The figure also indicates where the point scatterer in Fig. 3.2.1 falls in the parameter space. From Fig. 3.2.1b, the lateral PSF bounds appear at $x=3.593$ and $4.407 \mathrm{~mm}$, for a lateral resolution of $0.814 \mathrm{~mm}$. This is consistent with Fig. 3.2.2a, which shows a lateral resolution of 0.814 at $(3.99 \mathrm{~mm}, 14.99 \mathrm{~mm})$, the closest image point to the scatterer. Likewise, (3.2.1b) shows the axial PSF bounds at $z=14.77$ and $15.23 \mathrm{~mm}$, for an axial resolution of $0.46 \mathrm{~mm}$, which is consistent with Fig. 3.2.2b, which shows an axial resolution of $0.46 \mathrm{~mm}$ at $(3.99 \mathrm{~mm}, 14.99 \mathrm{~mm})$. Fig. 3.2.2b also shows blank regions near the array corresponding to image points for which (3.2.17) takes imaginary values. However, in SA tomography, it's more likely that objects to be imaged will be placed farther away from the array to be illuminated by the diverging beams. 


\subsubsection{Split-Step Fourier Back-Propagation}

The more general case of spatially-varying refractive index adds a degree of complexity as the $x$ and $y$ second derivatives in the PE operator (3.2.4) are coupled with the refractive index $n(\mathbf{r})$ by the square root operation. In order to make use of efficient fast Fourier transform (FFT) algorithms to carry out the back-propagation, these

derivative terms must be decoupled from the refractive index. This is typically done by successive Taylor expansions of the square root operator around a spatially-invariant reference refractive index, $n_{0}$. The first Taylor expansion yields

$$
Q(\mathbf{r})=\sqrt{(2 k)^{2} n_{0}^{2}+\frac{\partial^{2}}{\partial x^{2}}+\frac{\partial^{2}}{\partial y^{2}}}+\frac{(2 k)^{2} n_{0}}{\sqrt{(2 k)^{2} n_{0}^{2}+\frac{\partial^{2}}{\partial x^{2}}+\frac{\partial^{2}}{\partial y^{2}}}}\left[n(\mathbf{r})-n_{0}\right]+\cdots,
$$

the first term of which depends only on $n_{0}$. Decoupling the second term requires Taylor expanding it as well. Defining the refractive index perturbation

$$
\delta n(\mathbf{r}) \equiv n(\mathbf{r})-n_{0},
$$

we can make use of the Taylor series expansion

$$
\frac{1}{\sqrt{1+X}}=1-\frac{X}{2}+\cdots
$$


to rewrite the second term in $(3.2 .20)$ as

$$
\begin{aligned}
\frac{(2 k)^{2} n_{0} \delta n(\mathbf{r})}{\sqrt{(2 k)^{2} n_{0}^{2}+\frac{\partial^{2}}{\partial x^{2}}+\frac{\partial^{2}}{\partial y^{2}}}} & =\frac{2 k \delta n(\mathbf{r})}{\sqrt{1+\frac{1}{(2 k)^{2} n_{0}^{2}}\left[\frac{\partial^{2}}{\partial x^{2}}+\frac{\partial^{2}}{\partial y^{2}}\right]}} \\
& =2 k \delta n(\mathbf{r})-\frac{1}{2} \frac{2 k \delta n(\mathbf{r})}{(2 k)^{2} n_{0}^{2}}\left[\frac{\partial^{2}}{\partial x^{2}}+\frac{\partial^{2}}{\partial y^{2}}\right]+\cdots
\end{aligned}
$$

Keeping only the first term in the expansion of (3.2.23), the operator is given approximately by

$$
Q(\mathbf{r}) \approx \sqrt{(2 k)^{2} n_{0}^{2}+\frac{\partial^{2}}{\partial x^{2}}+\frac{\partial^{2}}{\partial y^{2}}}+2 k \delta n(\mathbf{r})
$$

Under these approximations, the spatially-varying refractive index term is completely decoupled from the spatial second derivative terms, which are easily applied in the spatial frequency domain using $-k_{x}^{2}$ and $-k_{y}^{2}$ as before in (3.2.8). This results in the final form of the SSF operator,

$$
\begin{aligned}
Q_{S S F}(\mathbf{r}) & \approx \sqrt{(2 k)^{2} n_{0}^{2}-k_{x}^{2}-k_{y}^{2}}+2 k \delta n(\mathbf{r}) \\
& =k_{z 0}+2 k \delta n(\mathbf{r}) .
\end{aligned}
$$

The SSF operator is accurate subject to the approximations made in its derivation, namely:

1. Propagation is weakly $z$-dependent, made in allowing the commutator term to be ignored in (3.2.5), resulting in (3.2.6). 
2. Refractive index perturbations are relatively small, allowing the $2^{\text {nd }}$-order and higher terms in (3.2.20) to be ignored.

3. Low angular frequencies, or equivalently, low lateral propagation angles, which makes the first term in (3.2.23) dominant.

\section{Implementation}

For practical implementation, the spatially-varying refractive index $n(\mathbf{r})=n(x, y, z)$ is separated into a laterally-invariant reference refractive index $n_{0}(z)$ and a laterallyvarying perturbation,

$$
\delta n(x, y) \equiv n(x, y, z)-n_{0}(z)
$$

as in (3.2.21). The two terms in (3.2.25) are then applied in two "split" steps to propagate the fields back a small distance $\Delta z$, over which the refractive index is assumed to be constant [32]:

Step 1: Propagate the fields from the $z$ plane to the $z+\Delta z$ plane by applying a phase shift corresponding to the first term in (3.2.25). This is carried out in the spatial frequency domain by first applying a 2D spatial Fourier transform in (3.1.16) along the $x$ and $y$ dimensions:

$$
\hat{\psi}\left(k_{x}, k_{y}, z+\Delta z, \omega\right)=\mathcal{F}_{x, y}\{\psi(x, y, z, \omega)\} e^{i k_{z 0}(z) \Delta z}
$$

Step 2: Apply a phase correction corresponding to the second term in (3.2.25) to the result of the previous step. This is carried out in the spatial domain by first applying a 2D inverse spatial Fourier transform in (3.1.17) along the $k_{x}$ and $k_{y}$ 
dimensions:

$$
\psi_{S S F}(x, y, z+\Delta z, \omega)=\mathcal{F}_{k_{x}, k_{y}}^{-1}\left\{\hat{\psi}\left(k_{x}, k_{y}, z+\Delta z, \omega\right)\right\} e^{i 2 k \delta n(x, y) \Delta z}
$$

After the fields have been back-propagated to the $z+\Delta z$ plane, the image intensity is calculated by integrating over temporal frequency as in (3.2.10), resulting in

$$
I_{S S F}(x, y, z)=\left|\frac{1}{2 \pi} \int_{-\infty}^{\infty} \psi_{S S F}(x, y, z, \omega) d \omega\right|^{2}
$$

The process is then repeated until a full 3D tomographic image is constructed.

From (3.2.26), it can be seen that for the special case of $n(x, y, z)=1,(3.2 .25)$ simplifies to (3.2.8) and split-step Fourier back-propagation reduces to Fourier backpropagation.

\section{Error Analysis: Propagation Step Size}

With reference to (3.2.7), the fields back-propagated from the $z$ plane to the $z+\Delta z$ plane using the exact operator (3.2.4) can be written as

$$
\psi_{Q}(x, y, z+\Delta z, \omega)=\psi(x, y, z, \omega) \exp \left(i \int_{z}^{z+\Delta z} Q(x, y, \zeta) d \zeta\right)
$$


Likewise, the fields back-propagated using the SSF propagator (3.2.25) can be written as

$$
\psi_{Q_{S S F}}(x, y, z+\Delta z, \omega)=\psi(x, y, z, \omega) \exp \left(i \int_{z}^{z+\Delta z} Q_{S S F}(x, y, \zeta) d \zeta\right)
$$

The relative error between $\psi_{Q}$ and $\psi_{Q_{S S F}}$ can therefore be written as

$$
\begin{aligned}
E_{\psi}(x, y, z+\Delta z, \omega) & \equiv \frac{\psi_{Q_{S S F}}(x, y, z+\Delta z, \omega)-\psi_{Q}(x, y, z+\Delta z, \omega)}{\psi_{Q}(x, y, z+\Delta z, \omega)} \\
& =\frac{\psi_{Q_{S S F}}(x, y, z+\Delta z, \omega)}{\psi_{Q}(x, y, z+\Delta z, \omega)}-1 \\
& =\exp \left(i \int_{z}^{z+\Delta z} Q_{S S F}(x, y, \zeta)-Q(x, y, \zeta) d \zeta\right)-1 .
\end{aligned}
$$

From the mean value theorem for definite integrals [139], there exists at least one set of points $z \leq \zeta(x, y) \leq z+\Delta z$ for which the integral satisfies

$$
i \int_{z}^{z+\Delta z} Q_{S S F}(x, y, \zeta)-Q(x, y, \zeta) d \zeta=\mu(x, y, \omega) \Delta z
$$

which allows rewriting (3.2.32) as

$$
E_{\psi}(x, y, z+\Delta z, \omega)=\exp (\mu(x, y, \omega) \Delta z)-1 .
$$


Expanding the exponential function to $3^{\text {rd }}$ order then yields

$$
\begin{aligned}
E_{\psi}(x, y, z+\Delta z, \omega)=\mu(x, y, \omega) & \Delta z \\
& +\frac{1}{2} \mu^{2}(x, y, \omega)(\Delta z)^{2}+\frac{1}{6} \mu^{3}(x, y, \omega)(\Delta z)^{3}+\cdots .
\end{aligned}
$$

The error in the propagated fields is therefore first order in $\Delta z$ [139]. Common treatments of the split-step Fourier method often introduce four alternative ways to split the operator either symmetrically or asymmetrically to achieve second or third order errors in $\Delta z$ at the expense of computational complexity (i.e. more Fourier transforms) [32]. The analysis here, which closely follows that in [139], demonstrates these alternatives provide no advantages in accuracy, as the error is always first order in $\Delta z$. The SSF back-propagation method described above is the least computationally expensive and was therefore chosen for this work.

In practice, a reasonable upper limit for $\Delta z$ is given by

$$
\Delta z_{\max }=\frac{\pi}{4 \max |k \delta n(x, y)|},
$$

which ensures that the phase correction applied in (3.2.28) doesn't introduce any phase changes greater than $\pi / 2$ radians. 


\section{Error Analysis: Propagation Angle and Refractive Index Perturbation}

For a medium characterized by an arbitrary, spatially-invariant refractive index $n$, the propagator in (3.2.4) can be expressed as

$$
\begin{aligned}
Q & =\sqrt{(2 k)^{2} n^{2}-k_{x}^{2}-k_{y}^{2}} \\
& =2 k n \cos \theta_{p}
\end{aligned}
$$

where $\theta_{p}$ is the propagation angle from the $z$-axis [139]. Similarly, the split-step Fourier propagator from $(3.2 .25)$ can be expressed as

$$
\begin{aligned}
Q_{S S F} & =\sqrt{(2 k)^{2} n_{0}^{2}-k_{x}^{2}-k_{y}^{2}}+2 k \delta n \\
& =\sqrt{(2 k)^{2} n_{0}^{2}-\sin ^{2} \theta_{p}}+2 k \delta n
\end{aligned}
$$

where $n=n_{0}+\delta n$ as in (3.2.26). Using (3.2.37) and (3.2.38), the relative propagator error can then be quantified in terms of the propagation angle $\theta_{p}$ and relative refractive index perturbation $\delta n / n_{0}$ as [139]

$$
\begin{aligned}
E_{Q}\left(\theta_{p}, \delta n / n_{0}\right) & \equiv \frac{Q_{S S F}-Q}{Q}=\frac{Q_{S S F}}{Q}-1 \\
& =\frac{\sqrt{\left(1+\delta n / n_{0}\right)^{-2}-\sin ^{2} \theta_{p}}+\frac{\delta n / n_{0}}{1+\delta n / n_{0}}}{\cos \theta_{p}}-1 .
\end{aligned}
$$

Under the tangent plane approximation, a sensor in the array plane at $x_{0}$ as shown in Fig. 3.2.3 will receive a specular return from the curved surface of radius $a$ at an angle of $\theta_{p}$ to the $z$-axis. The figure also shows that $\theta_{p}$ is equal to the angle of 


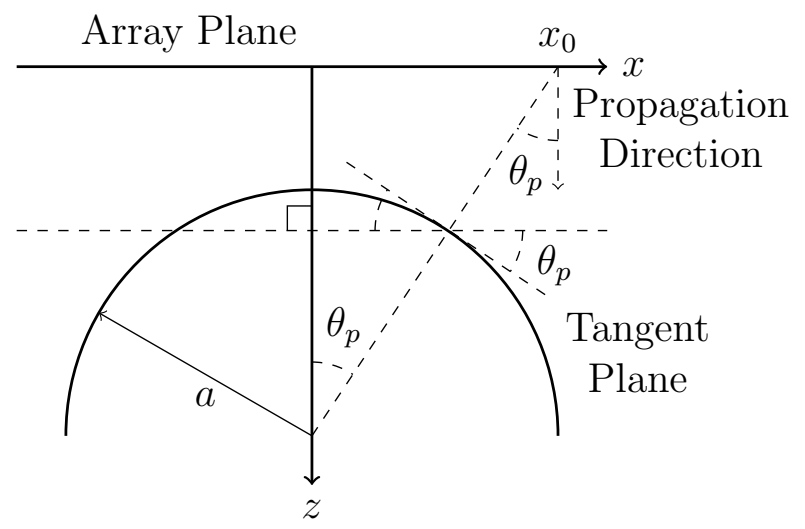

Figure 3.2.3: Diagram illustrating the tangent plane approximation, under which a sensor at $x_{0}$ in the array plane will receive a specular reflection from the curved surface at an angle $\theta_{p}$ from the $z$-axis, which is equal to the tilt angle of the tangent plane.

tilt of the tangent plane. Fig. 3.2.4 shows a contour plot of the relative propagator error in (3.2.39) calculated over a range of propagation angles and refractive index perturbations. As will be shown in Sec. 4.3, this relative propagator error in (3.2.39), combined with the observation from Fig. 3.2.3 that the propagation angle $\theta_{p}$ is equal to the tangent plane tilt angle allows the formulation of simulation test scenarios that enable the investigation of the influence of approximation error on thickness estimates from SSF-processed image data. 


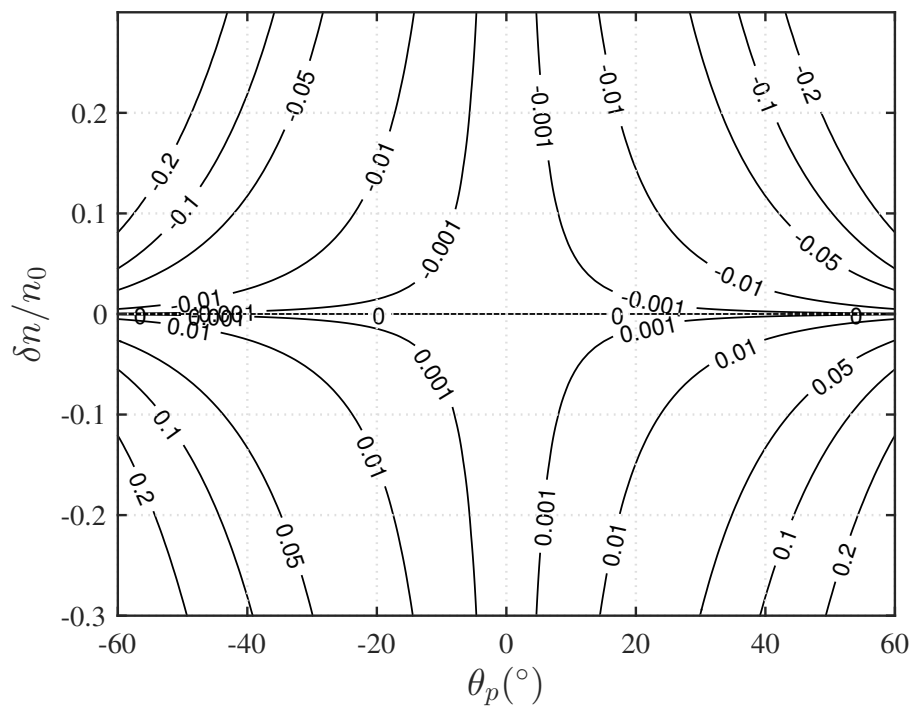

Figure 3.2.4: Relative error in the SSF propagator (3.2.39) as a function of propagation angle $\theta_{p}$ and relative refractive index perturbation $\delta n / n_{0}$. 


\section{Chapter 4}

\section{Implementation, Demonstration, and Analysis}

This chapter presents details on the implementation of the $\mathrm{THz}$ imaging methods described in Chapter 3 using the Picometrix T-Ray $4000 \mathrm{THz}$ imaging system at the Northwest Electromagnetics and Acoustics Research Laboratory (NEAR-Lab). Experimental results demonstrating the ability of the SSF back-propagation method to generate accurate $3 \mathrm{D} \mathrm{THz} \mathrm{tomographic} \mathrm{images} \mathrm{of} \mathrm{a} \mathrm{pharmaceutical} \mathrm{tablet} \mathrm{as} \mathrm{well}$ as more well-characterized test objects are presented, followed by simulation results that quantify and characterize the influence of approximation error in the SSF backpropagation method on thickness estimates of a dielectric layer. Results shown in this chapter were presented at the 2013 SPIE Optics and Photonics conference in San Diego, CA [39]; the 2015 International Radar Symposium (IRS) in Dresden, Germany [40]; and were included in a manuscript recently submitted for publication in the IEEE Transactions on Terahertz Science and Technology [41]. 


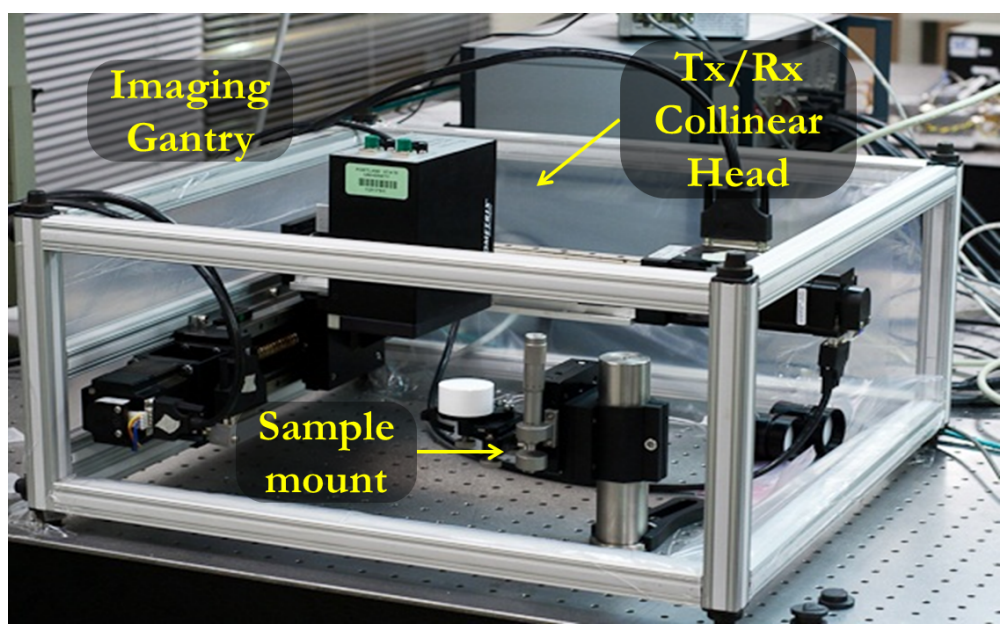

Figure 4.1.1: Picometrix T-Ray $4000 \mathrm{THz}$ time-domain imaging system.

\subsection{Picometrix T-Ray 4000 Imaging System}

While phased-array methods can be applied to data collected using physical sensor arrays, such as those depicted in Fig. 1.5, potentially in multistatic configurations, the measurements presented in this work employ the Picometrix T-Ray $4000 \mathrm{THz}$ time-domain imaging system shown in Fig. 4.1.1 - a convenient experimental testbed for the imaging methods introduced in Sec. 3.2. The T-Ray 4000 records a 4096point waveform every $10 \mathrm{~ms}$. The internal delay line allows a time-domain sampling interval of $78.125 \mathrm{ps}$, for a Nyquist frequency of $6.4 \mathrm{THz}$. As the zero-mean random noise is uncorrelated from one waveform to the next, multiple waveforms are typically averaged to increase signal-to-noise ratio (SNR). In the imaging setup shown in Fig. 4.1.1, both the transmitting and receiving antennas are contained in the collinear head, which uses a beam splitter to allow monostatic measurements through a common high-density-polyethylene (HDPE) focusing lens. In addition to the random noise reduced by averaging multiple waveforms, the beam splitter, lenses, and other internal components introduce a coherent background waveform that must be record- 


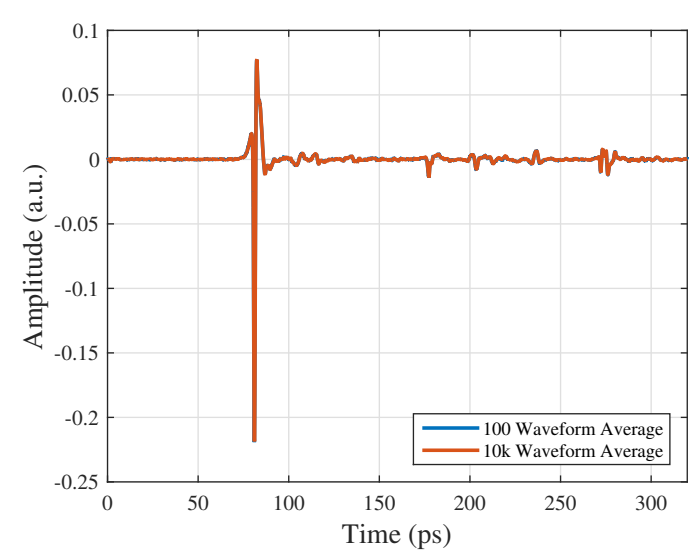

(a)

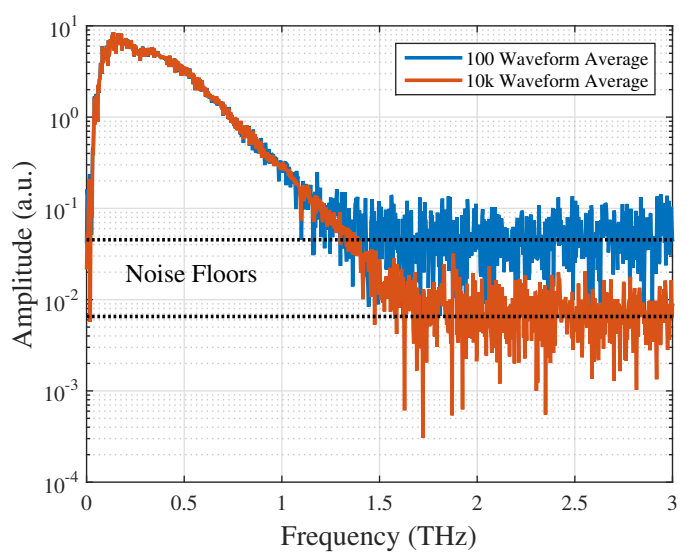

(b)

Figure 4.1.2: Example waveforms and amplitude spectra generated using T-Ray 4000 operating in monostatic reflection mode with a $1^{\prime \prime}$ focal length lens. Waveforms shown in (a) are the coherent average of 100 and 10,000 waveforms. Coherent averaging in this way increases signal-to-noise ratio. This is more evident in (b), which shows the corresponding Fourier amplitude spectra on a log scale. As the figure shows, the noise floor is relatively flat across the $\mathrm{THz}$ spectrum.

ed separately (with significant averaging to minimize the reintroduction of random noise) and subtracted from the measured data prior to use in image processing. Two example waveforms generated in this manner are shown in Fig. 4.1.2a. These waveforms were obtained using the collinear head, equipped with a $1^{\prime \prime}$ focal length HDPE lens to focus the $\mathrm{THz}$ pulses onto a gold reference mirror. The waveforms shown are the coherent average of 100 and 10,000 waveforms. The resulting increase in SNR is most evident in the Fourier amplitude spectra, obtained via FFT of the time-domain waveforms and shown on a log scale in Fig. 4.1.2b. As the figure shows, the noise floor is easily identifiable and relatively flat across the THz spectrum. The dashed lines indicating the noise floors were generated by incoherently averaging the amplitudes of the frequency components above $2 \mathrm{THz}$.

As synthetic aperture methods require a diverging beam, the $1^{\prime \prime}$ focal length HDPE lens is employed to effect a point-like "virtual transceiver" at the lens's focal point 


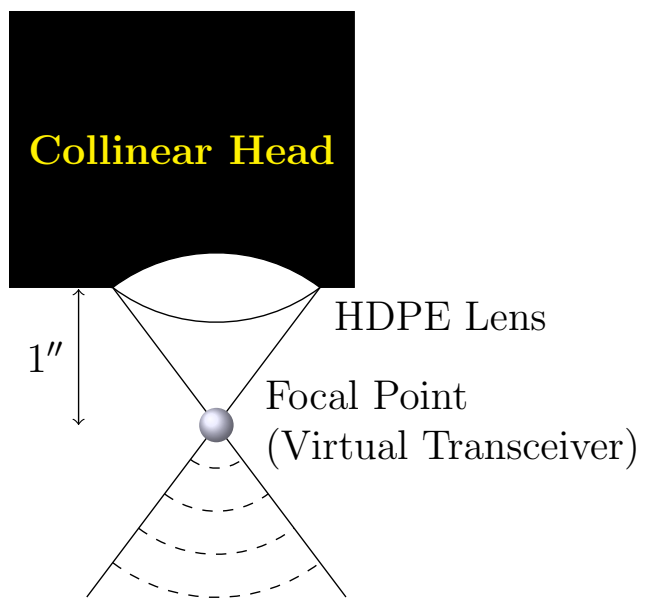

Phase Fronts

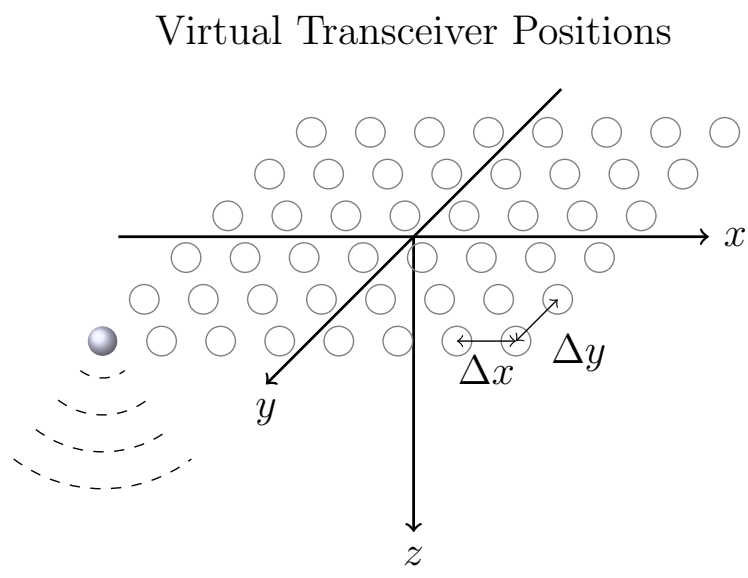

(b)

Figure 4.1.3: (a) Point-like virtual transceiver at focal point of $1^{\prime \prime}$ lens [91]. (b) Synthetic array formed by raster scanning virtual transceiver laterally in the $x y$-plane $[23,24]$.

below the collinear head, as depicted in Fig. 4.1.3a [23, 24, 39, 91]. The computercontrolled imaging gantry then allows the virtual transceiver to be raster scanned laterally in the $x y$ plane as illustrated in Fig. $4.1 .3 \mathrm{~b}$, collecting data much like in stripmap-mode SAR. The array spacing $\Delta x$ and $\Delta y$ are set by the $\lambda / 4$ spatial Nyquist limit for monostatic synthetic aperture measurements as described in Sec. 3.1.

Two additional measurements must be made for each $\mathrm{THz}$ SA image: a measurement of the background waveform described above and a reference measurement with the gold mirror like those shown in Fig. 4.1.2. The planar surface of the gold mirror used in the reference measurement is also used to align the sample stage with the imaging gantry's translation in $x$ and $y$ prior to scanning. As the background waveform is common to both the SA data and the reference waveform, it is used to correct for drift in the T-Ray 4000's delay line. This is done by aligning the SA and reference data with the background waveform prior to subtraction. After the background subtraction, 
the reference waveform is used for preprocessing the measured data using methods described in the following section.

\subsubsection{Waveform Preprocessing}

The reference waveform measured from the reflective mirror can be expressed as [22]

$$
w_{r}(t)=\psi_{i}(t)+n_{r}(t)
$$

where $\psi_{i}(t)$ is the incident waveform and $n_{r}(t)$ is the additive zero-mean Gaussian noise in the reference measurement. In many Terahertz pulsed imaging and spectroscopy applications, the sample under test is assumed to behave like a linear

time-invariant system with impulse response $h(t)$, which acts to generate delayed and attenuated copies of the transmitted waveform [22]. The resulting waveform recorded in the sample measurement can be expressed as a convolution of the incident pulse with the sample's impulse response,

$$
w_{s}(t)=h(t) * \psi_{i}(t)+n_{s}(t)
$$

where $n_{s}(t)$ is additive zero-mean Gaussian noise in the sample measurement. In a typical SA imaging experiment, the reference waveform used in preprocessing is the average of 10,000 waveforms, whereas the sample measurement is the average of only 100 or so waveforms. The reference measurement therefore has a much $(100 \times)$ higher signal-to-noise ratio (SNR) than the sample measurement over the band of interest, as shown in Fig. 4.1.2b. In this case, the reference measurement provides a good estimate of the transmitted waveform and can be used as such in preprocessing of the 
sample waveforms.

One common preprocessing method comes from signal processing in radar and active sonar, in which received signals are cross-correlated with the known transmitted waveform to suppress noise and decrease the temporal pulse width in a process commonly referred to as pulse compression or matched filtering [140]. In the preprocessing of $\mathrm{THz}$ signals, the known transmitted waveform is given by the reference waveform [141] and the matched filter's transfer function is given in the frequency domain by

$$
b_{\mathrm{MF}}(\omega)=w_{r}^{*}(\omega)
$$

where the superscript "*” denotes complex conjugation. This conjugation results in a simple cross-correlation of the sample and reference waveforms when the matched filter is applied.

In contrast to matched filtering, preprocessing of the waveforms often involves normalizing the data by the incident spectrum as described in Sec. 3.1. This method, often referred to as deconvolution, attempts to estimate $h(t)$ given the reference and sample measurements [22]. Taking the ratio of $w_{r}(t)$ and $w_{s}(t)$ in the frequency domain yields

$$
\frac{w_{s}(\omega)}{w_{r}(\omega)}=\frac{h(\omega) \psi_{i}(\omega)}{\psi_{i}(\omega)+n_{r}(\omega)}+\frac{n_{s}(\omega)}{\psi_{i}(\omega)+n_{r}(\omega)} .
$$

As the reference measurement typically has a much higher signal-to-noise ratio (SNR) than the sample measurement over the band of interest, $\psi_{i}(\omega)>>n_{r}(\omega)$ and (4.1.4) can be approximated as

$$
\frac{w_{s}(\omega)}{w_{r}(\omega)} \approx h(\omega)+\frac{n_{s}(\omega)}{\psi_{i}(\omega)}
$$

If the SNR in the sample measurement is sufficiently high (i.e., $\left.|h(\omega)|>>\frac{n_{s}(\omega)}{\psi_{i}(\omega)}\right)$, 
(4.1.5) provides a good estimate of the transfer function $h(\omega)$. However, for large $\left|n_{s}(\omega)\right|$ or small $\left|\psi_{i}(\omega)\right|$, the noise term dominates and the result becomes unreliable. In most pulsed $\mathrm{THz}$ time domain systems, SNR decays with increasing frequency as shown in Fig. 4.1.2b, so various low-pass filters, such as the double Gaussian (DG) or customized skewed Gaussian (CSG) filters are often applied to (4.1.5) to suppress the noisy parts of the spectrum [22]. Another standard approach is to use the Wiener deconvolution filter,

$$
b_{\mathrm{WD}}(\omega)=\frac{w_{r}^{*}(\omega)}{\left|w_{r}(\omega)\right|^{2}+\left|n_{s}(\omega)\right|^{2}},
$$

which provides the minimum mean square estimate of the transfer function in the presence of additive noise $[22,142]$. As the numerator of the Wiener deconvolution filter is in fact the matched filter (4.1.3), the two filters have an identical phase response [141].

The behavior of the Wiener deconvolution filter in (4.1.6) can be analyzed by expressing it as the product of a deconvolution filter and a de-noising filter, i.e.

$$
\begin{aligned}
b_{\mathrm{WD}}(\omega) & =b_{\text {deconv. }}(\omega) b_{\text {de-noise }}(\omega) \\
& =\frac{1}{w_{r}(\omega)} \frac{\left|w_{r}(\omega)\right|^{2}}{\left|w_{r}(\omega)\right|^{2}+\left|n_{s}(\omega)\right|^{2}} .
\end{aligned}
$$

The de-noising filter can be rewritten as

$$
\begin{aligned}
b_{\text {de-noise }}(\omega) & =\frac{\left|w_{r}(\omega)\right|^{2}}{\left|w_{r}(\omega)\right|^{2}+\left|n_{s}(\omega)\right|^{2}} \\
& =\frac{1}{1+\left|\frac{n_{s}(\omega)}{w_{r}(\omega)}\right|^{2}} .
\end{aligned}
$$

From this expression, it can be seen that for frequency components with low SNR, 
$1<<\left|n_{s}(\omega) / w_{r}(\omega)\right|^{2}$ in the denominator, and (4.1.7) reduces to

$$
b_{W D}(\omega) \approx \frac{1}{w_{r}(\omega)}\left|\frac{w_{r}(\omega)}{n_{s}(\omega)}\right|^{2} .
$$

The deconvolution filter is therefore weighted by the (low) SNR, which suppresses the output. Conversely, for frequency components with high SNR, $1>>\left|n_{s}(\omega) / w_{r}(\omega)\right|^{2}$ in the denominator, reducing (4.1.8) to unity and (4.1.7) to the simple deconvolution filter,

$$
b_{W D}(\omega) \approx \frac{1}{w_{r}(\omega)}
$$

For the intermediate case of unit SNR, $\left|w_{r}(\omega) / n_{s}(\omega)\right|^{2}=1$ in the denominator and (4.1.7) reduces to

$$
b_{W D}(\omega) \approx \frac{1}{w_{r}(\omega)} \frac{1}{2}
$$

This shows that while Weiner deconvolution provides the optimal trade off (in the least squares sense) between deconvolution and noise suppression, the de-noising term only suppresses the output by a factor of 2 for signals at the noise floor.

In practice, Wiener deconvolution is often implemented as

$$
b_{\mathrm{P}}(\omega)=\frac{w_{r}^{*}(\omega)}{\left|w_{r}(\omega)\right|^{2}+\eta},
$$

where the regularization parameter $\eta$ is "tuned" manually to achieve a balance between deconvolution and de-noising. A simple, intuitive parameterization of this filter can be obtained by selecting $\eta$ such that the de-noising filter component applies a selectable level of attenuation $\alpha$ to frequency components at a cutoff power level $P_{c}(\omega)$, i.e.

$$
b_{\text {de-noise }}(\omega)=\frac{P_{c}(\omega)}{P_{c}(\omega)+\eta}=\frac{1}{\alpha} \text {. }
$$




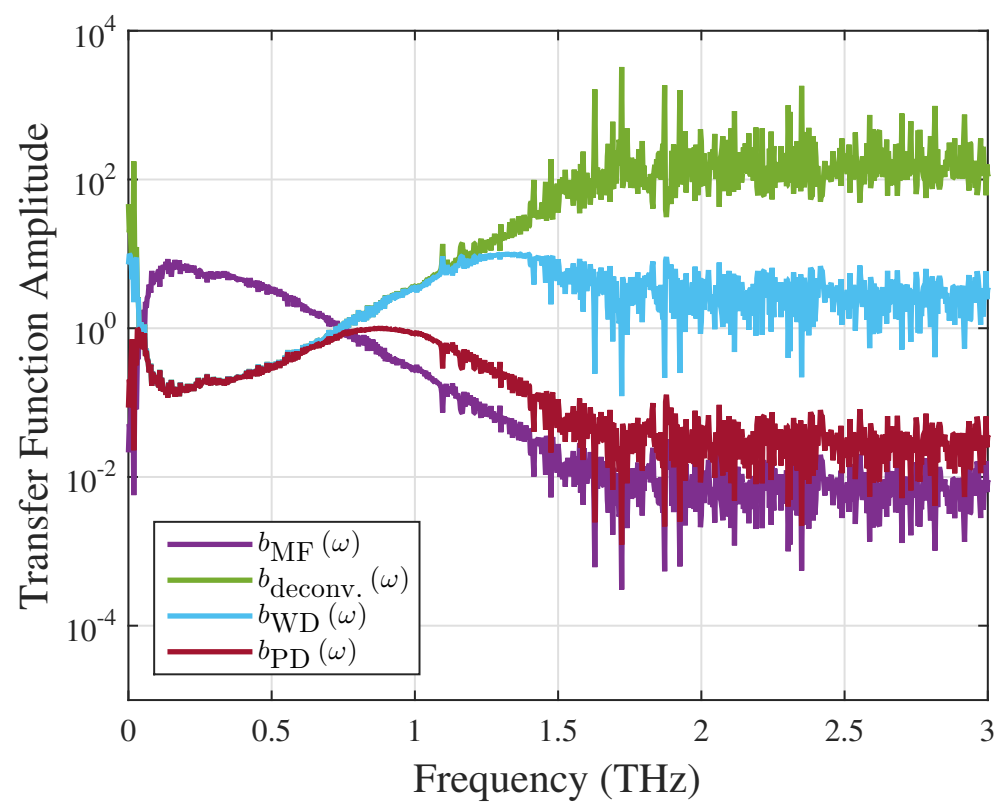

Figure 4.1.4: Comparison of the amplitude response of the matched filter $b_{\mathrm{MF}}(\omega)$ from (4.1.3), the deconvolution filter $b_{\text {deconv. }}(\omega)$ from (4.1.7), the Wiener deconvolution filter $b_{\mathrm{WD}}(\omega)$ from (4.1.6) and the parameterized Wiener deconvolution filter $b_{\mathrm{PD}}(\omega)$ in $(4.1 .14)$, where $w_{r}(\omega)$ is given by the 10,000 reference waveform average in Fig. 4.1.2. For $b_{\mathrm{PD}}(\omega), \alpha=100$ and the power cutoff level was set to the noise floor in the 100-waveform average in Fig. 4.1.2

Solving for $\eta$ and substituting into (4.1.12) results in

$$
b_{\mathrm{PD}}(\omega)=\frac{w_{r}^{*}(\omega)}{\left|w_{r}(\omega)\right|^{2}+(\alpha-1) P_{c}(\omega)} .
$$

For the special case of $\alpha=2$ and $P_{c}(\omega)=\left|n_{s}(\omega)\right|^{2}$, this filter simplifies to the classical Wiener deconvolution filter (4.1.6).

Fig. 4.1.4 shows a comparison of the amplitude response of the matched filter $b_{\mathrm{MF}}(\omega)$ from (4.1.3), the deconvolution filter $b_{\text {deconv. }}(\omega)=1 / w_{r}(\omega)$ from (4.1.7), the Wiener deconvolution filter $b_{\mathrm{WD}}(\omega)$ from (4.1.6) and the parameterized Wiener deconvolution filter $b_{\mathrm{PD}}(\omega)$ in (4.1.14). The reference waveform $w_{r}(\omega)$ used in this analysis is the 10,000-waveform average shown in Fig. 4.1.2. For the parameterized Wiener 
deconvolution, $\alpha=100$ and the power cutoff level was set to the noise floor in the 100-waveform average in Fig. 4.1.2. The figure shows that the matched filter acts to suppress any frequency components that weren't sent out by the system while the deconvolution filter acts to "whiten" the signal by flattening the frequency spectrum over the entire band, amplifying the noise floor in the process. In contrast, the Wiener deconvolution filters flatten the frequency spectrum where the SNR is high and suppress the output where the SNR is low. This whitening effectively increases the bandwidth of the THz pulses, which improves the range resolution (3.2.11) of the $\mathrm{THz}$ imaging system. This can be demonstrated by applying these transfer functions to the 100-waveform averaged signal $w_{100}(t)$ in Fig. 4.1 .2 and taking the squared envelope of the resulting analytic signals, i.e.

$$
\hat{h}(t)=\left|\left(w_{100}(t)+\mathcal{H}\left\{w_{100}(t)\right\}\right) * b(t)\right|^{2},
$$

where $\mathcal{H}\{\cdot\}$ indicates a Hilbert transform and the impulse response $b(t)$ corresponds to the transfer functions shown in Fig. 4.1.4. The normalized output of (4.1.15) for each filter shown in Fig. 4.1.4 is shown in Fig. 4.1.5. As the figure shows, the matched filter does no whitening of the signal and therefore produces the broadest pulse $(\tau \approx 1.8 \mathrm{ps})$ and coarsest range resolution $(\delta r \approx 0.27 \mathrm{~mm})$. As expected, the deconvolution filter acts to whiten the entire signal spectrum, amplifying even the noisiest frequency components and obscuring the pulse altogether. The Wiener deconvolution outputs both show narrower pulses than the matched filter output and a reduction in noise over the deconvolution filter. Specifically, the FWHM pulse widths $\tau$ were $\approx 0.94$ and $0.55 \mathrm{ps}$, corresponding to range resolutions $\delta r \approx 0.140$ and $0.083 \mathrm{~mm}$ for $b_{\mathrm{WD}}(\omega)$ and $b_{\mathrm{PD}}(\omega)$, respectively. These results show how the range resolution can be improved with preprocessing, however the amplitude in most 


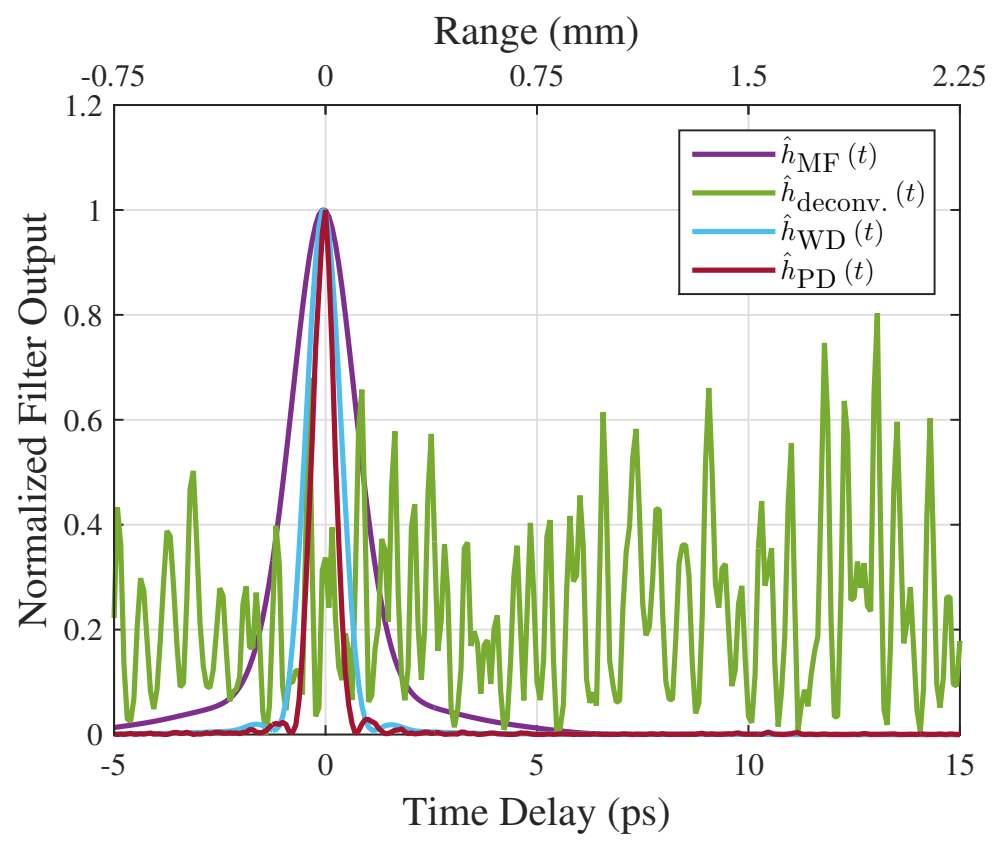

Figure 4.1.5: Comparison of the normalized output of (4.1.15), whose impulse responses correspond to the transfer functions are shown in Fig. 4.1.4.

sample signals $w_{s}(t)$ will be much lower than $w_{100}(t)$ in Fig. 4.1.2, so the actual gains in resolution may be less than the above analysis indicates.

\subsection{Experimental Results}

This section presents results from three experiments conducted using the Picometrix T-Ray 4000 described in the previous section. The first experiment, described in Sec. 4.2.1, features measurement results from an over the counter pharmaceutical gelcap and is presented as a "proof of concept." As the constituent materials of the gelcap are unknown, two further experiments were conducted on well-characterized test samples to demonstrate and quantify the accuracy of the images produced using SSF back-propagation. The first of these experiments, described in Sec. 4.2.2, involved a cylindrically-curved high-density polyethylene sample with high-contrast copper 


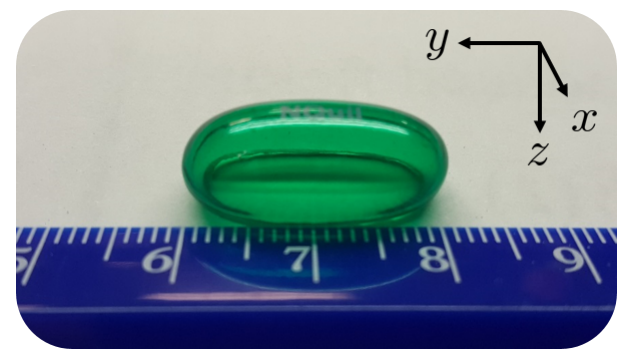

(a)

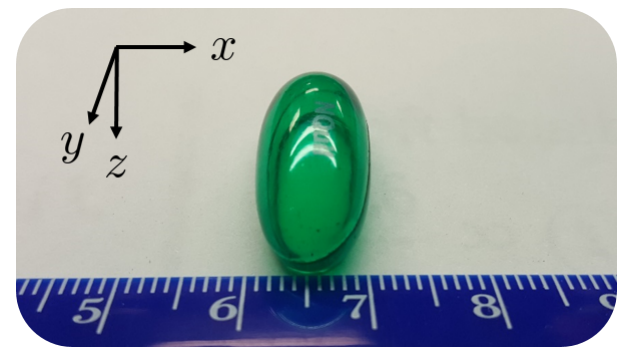

(b)

Figure 4.2.1: Over-the-counter gelcap tablet imaged using the Picometrix T-Ray 4000 imaging system described in Sec. 4.1. Emptying the gelcap of its liquid contents was found to enhance the $\mathrm{THz}$ reflection from the inner surface.

wires embedded within. Results from this experiment were presented at the 2013 SPIE Optics and Photonics conference [39] in San Diego, CA. The second of these experiments, described in Sec. 4.2.3, was conducted on a specially-designed 3D-printed test object. Results from this experiment were presented at the 2015 International Radar Symposium (IRS) in Dresden, Germany [40] and were included in a manuscript recently submitted for publication in the IEEE Transactions on Terahertz Science and Technology [41].

\subsubsection{Pharmaceutical Tablet}

This section describes an experiment carried out as a "proof of concept," which employed the over-the-counter gelcap tablet shown in Fig. 4.2.1. Emptying the gelcap of its liquid contents was found to enhance the $\mathrm{THz}$ reflection from the inner surface. As shown in the figure, the gelcap is ellipsoidal, approximately $2 \mathrm{~cm}$ long along the $y$ direction and with a maximum diameter of $\approx 1 \mathrm{~cm}$ in the $x z$-plane. The hollow gelcap was imaged using the Picometrix T-Ray $4000 \mathrm{THz}$ time domain system to form a $300 \times 300$-element planar synthetic aperture approximately $15 \mathrm{~mm}$ above the 

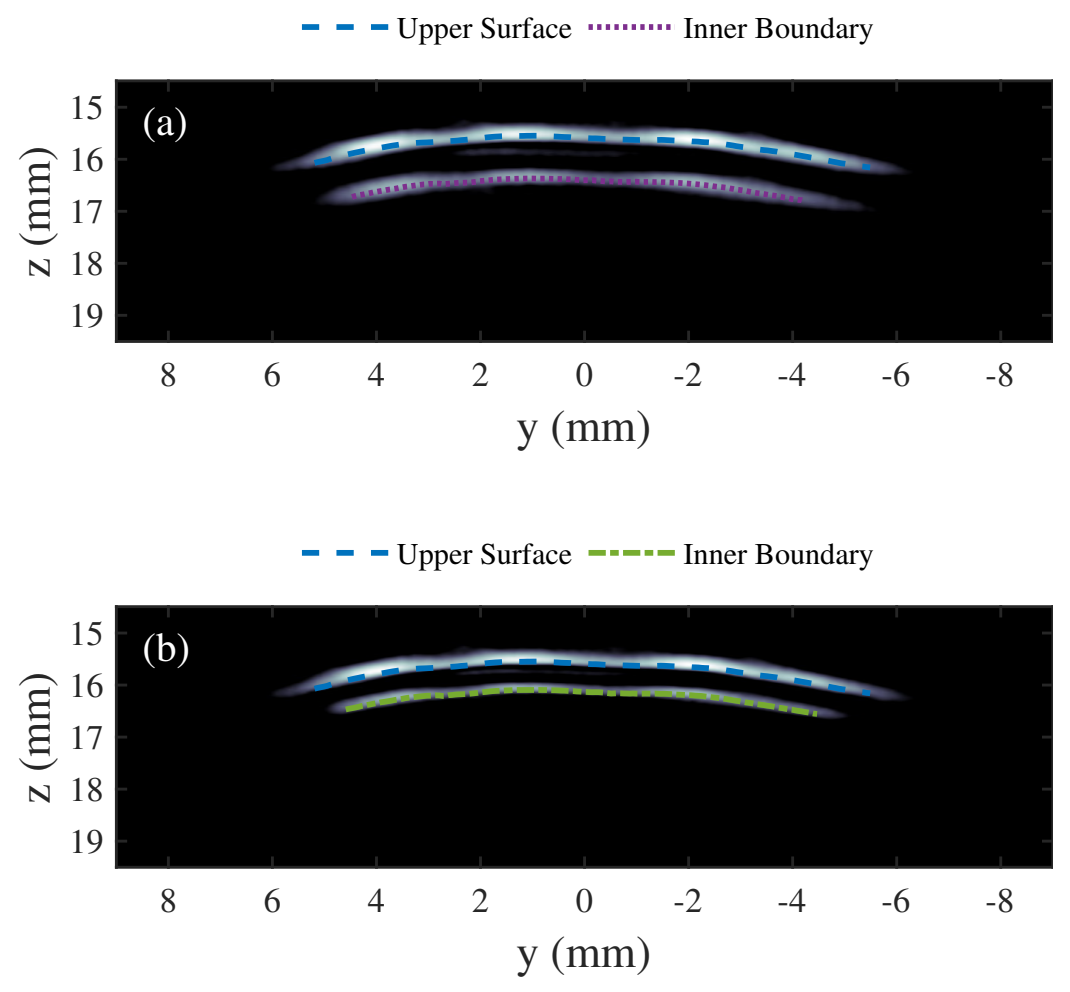

Figure 4.2.2: Axial slices through the FBP-processed (a) and SSF-processed (b) 3D image data along with the depths of the top boundary and inner boundaries.

sample as described in Sec. 4.1. The array spacing in $x$ and $y$ was kept to $0.060 \mathrm{~mm}$ to satisfy $\lambda / 4$ spatial sampling up to $\approx 1.25 \mathrm{THz}$, and 100 waveforms were averaged at each sensor location to increase SNR.

After preprocessing the waveforms using the modified Wiener deconvolution described in Sec. 4.1.1, the data were processed using FBP as described in Sec. 3.2.1 with depth resolution $\Delta z=12.7 \mu \mathrm{m}$, corresponding to $1 / 8^{\text {th }}$ of the upper limit in $(3.2 .36)$. Fig. 4.2.2 shows a cross-sectional slice through the resulting 3D image data along the axis of the gelcap, corresponding to the orientation in Fig. 4.2.1a. As described in Sec. 3.2.1, the 3D image resulting from FBP is used to localize the object's upper surface boundary, shown as a dashed purple line in Fig. 4.2.2a along with the apparent 
location of the inner boundary, shown as a dotted green line.

Having localized the object's upper boundary using the FBP-processed 3D image, the data can be reprocessed using the SSF method as described in Sec. 3.2.2, provided the refractive index of the object under test is known. Mismatch between the actual and assumed refractive index used in SSF back-propagation will introduce a proportional mismatch between the actual and apparent vertical location of the inner boundary. The limiting case of this mismatch, in which free space is assumed throughout the image volume, is represented by FBP processing. Therefore, SSF processing using any assumed refractive index between unity (free space) and the actual refractive index will produce a more accurately reconstructed image than FBP processing. Likewise, if a refractive index greater than the actual refractive index is assumed, the SSF processing will over-correct for the refraction, resulting in a less accurate image. In the case of the gelcap in this experiment, the refractive index of the sample was not known and was therefore assumed to be 1.53; a value taken from the literature [1]. Fig. 4.2.2b shows a cross-sectional slice through the resulting SSF-processed image data at the same location as Fig. 4.2.2a. The figure also shows the apparent location of the inner boundary in the SSF-processed image data.

Fig. 4.2.2 shows the layer appears to be thinner in the SSF-processed data than in the FBP-processed data, as is to be expected due to refraction within the sample. Specifically, the layer's radial thickness (mean \pm standard deviation) was found to be $0.792 \pm 0.025 \mathrm{~mm}$ for the FBP-processed data compared to the $0.520 \pm 0.018 \mathrm{~mm}$ for the SSF-processed data. These numbers are consistent with the radial thickness of $0.484 \pm$ $0.009 \mathrm{~mm}$ obtained from micrometer measurements of a separate gelcap from the same lot as that shown in Figs. 4.2.1 and 4.2.2. However, without an accurate estimate of 


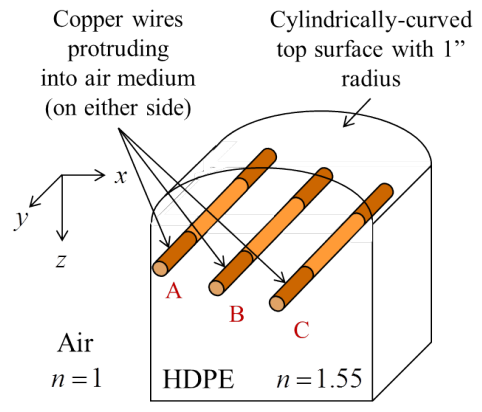

(a)

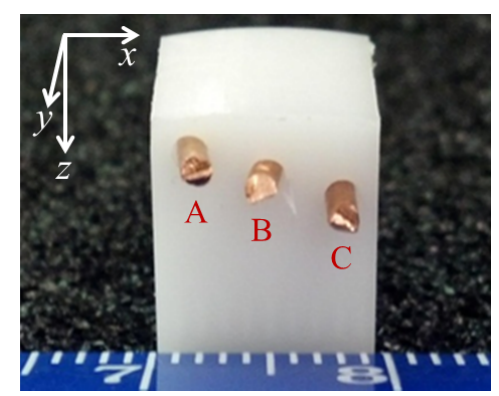

(b)

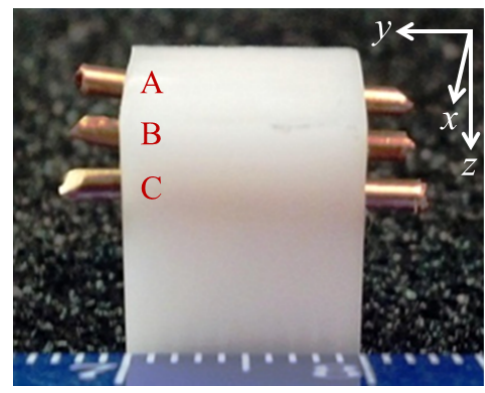

(c)

Figure 4.2.3: HDPE test object with cylindrically-curved top surface and protruding copper wires A, B, and C. (a) Diagram of test object. (b) $x z$-view showing curved top surface. (c) yz-view showing protruding copper wires.

the gelcap's refractive index, it is impossible to determine whether the difference in the thickness estimates is due to refractive index mismatch in the SSF processing, an actual difference in thickness between the two gel caps, or the approximation error in the SSF back-propagation described in Sec. 3.2.2. The following sections present results of experiments designed specifically to disentangle these sources of error.

\subsubsection{High-Density Polyethylene Test Object}

This section describes results from an experiment designed to demonstrate the ability of SSF back-propagation to correctly localize defects within a dielectric object with a curved surface. These results were presented at the 2013 SPIE Optics and Photonics conference in San Diego, California in August 2013 and published in the conference proceedings [39].

The dielectric test object, shown in Fig. 4.2.3, was fabricated from a $2^{\prime \prime}$-diameter highdensity polyethylene (HDPE) rod. HDPE is commonly used in THz lenses and other 
$\mathrm{THz}$ optical components due to its transparency and relatively constant refractive index of 1.55 across the $\mathrm{THz}$ band. The rod was cut and milled down to a roughly $1 \mathrm{~cm}^{3}$ cube with a cylindrically-curved top surface with a $1^{\prime \prime}(2.54 \mathrm{~cm})$ radius of curvature. Internal "defects" were introduced by drilling three holes through which copper wires were fed as shown in Fig. 4.2.3. The ends of the copper wires were left protruding to provide a "ground truth" of the wires' actual location. The wires were designated wire A, wire B, and wire $\mathrm{C}$, as indicated in Fig. 4.2.3, with wire A being closest to the curved top surface, wire C being farthest from the top surface, and wire $\mathrm{B}$ at an intermediate distance from the top surface. The object was then imaged using the T-Ray 4000 imaging system described in Sec. 4.1 to synthesize a $200 \times 200$ synthetic array approximately $10 \mathrm{~mm}$ above the object. The synthetic array elements spaced $0.1 \mathrm{~mm}$ in $x$ and $y$, corresponding to a $\lambda / 4$ spacing up to $\approx 0.75 \mathrm{THz}$.

FBP was then applied to the measured data to generate a 3D THz SA image consisting of 200 slices with $z$ values ranging from 8.2 to $17.6 \mathrm{~mm}$ below the array, a full 3D rendering of which is shown in Fig. 4.2.4. The curved surface is clearly resolved, as are the protruding ends of wires $\mathrm{A}, \mathrm{B}$, and $\mathrm{C}$. Wire returns from within the HDPE are attenuated relative to those from the protruding ends due to transmission and reflection losses through the HDPE surface. The shapes of the wires are also distorted within the object due to the HDPE's refractive index causing the segments of the wires inside the HDPE volume to appear farther below the surface than the ends protruding into free space.

The 3D location of the curved top surface boundary was obtained using the 3D image data shown in Fig. 4.2.4, after which the data were reprocessed using SSF back-propagation assuming $\operatorname{HDPE}(n=1.55)$ in the space below the profiled surface 

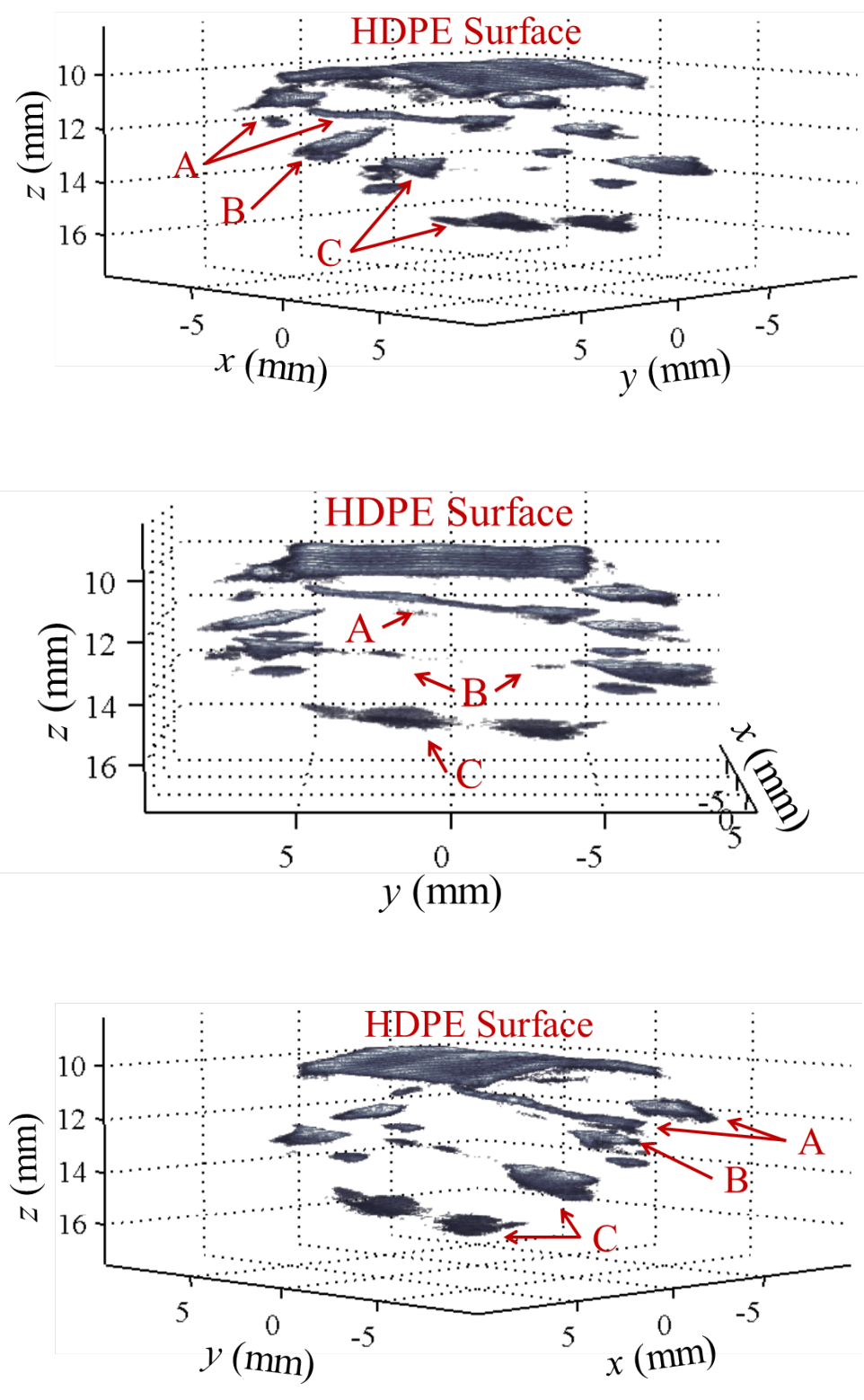

Figure 4.2.4: Volume rendering of $\mathrm{THz} \mathrm{SA}$ tomographic data of HDPE test object obtained using FBP shown at 3 different angles. Returns from the curved HDPE surface and wires A, B, and C (see Fig. 4.2.3) are labeled. 
as in Fig. 4.2.3a. The resulting image data are shown in Fig. 4.2.5. The curved surface and protruding wire ends are again clearly resolved, however the images of the wires appear to be continuous, unlike in Fig. 4.2.4.

This is emphasized in Fig. 4.2.6, which shows a side-by-side comparison of crosssectional slices through the volumetric data produced by both methods. The slices are taken at $x=-3.65 \mathrm{~mm},-0.45 \mathrm{~mm}$, and $2.75 \mathrm{~mm}$, corresponding to the locations of wires A, B, and C, respectively. Fig. 4.2.6a shows slices through the volumetric data in Fig. 4.2.4, obtained using FBP. As in Fig. 4.2.4, the apparent locations of the wires within the HDPE are displaced, as indicated by the arrows. The correct locations of the wires (i.e., where the wire images would be had no distortion occurred) are indicated by the dotted lines that connect the images of the protruding wire ends. Fig. 4.2.6b shows slices through the volumetric data in Fig. 4.2.5, resulting from SSF back-propagation. As in Fig. 4.2.5, the downward shift of the apparent wire locations (shown in Fig. 4.2.6a) has been largely corrected for by SSF back-propagation, resulting in a higher-fidelity image (i.e. the internal structures are correctly localized).

\subsubsection{D-Printed Test Object}

This section describes results from an experiment designed to demonstrate the ability of SSF back-propagation to correctly estimate the thickness of a curved dielectric layer. These results were presented at the 2015 International Radar Symposium (IRS) in Dresden, Germany in June 2015 and published in the conference proceedings [40]. They are also featured in a manuscript recently submitted for publication in the IEEE Transactions on THz Science and Technology [41]. 

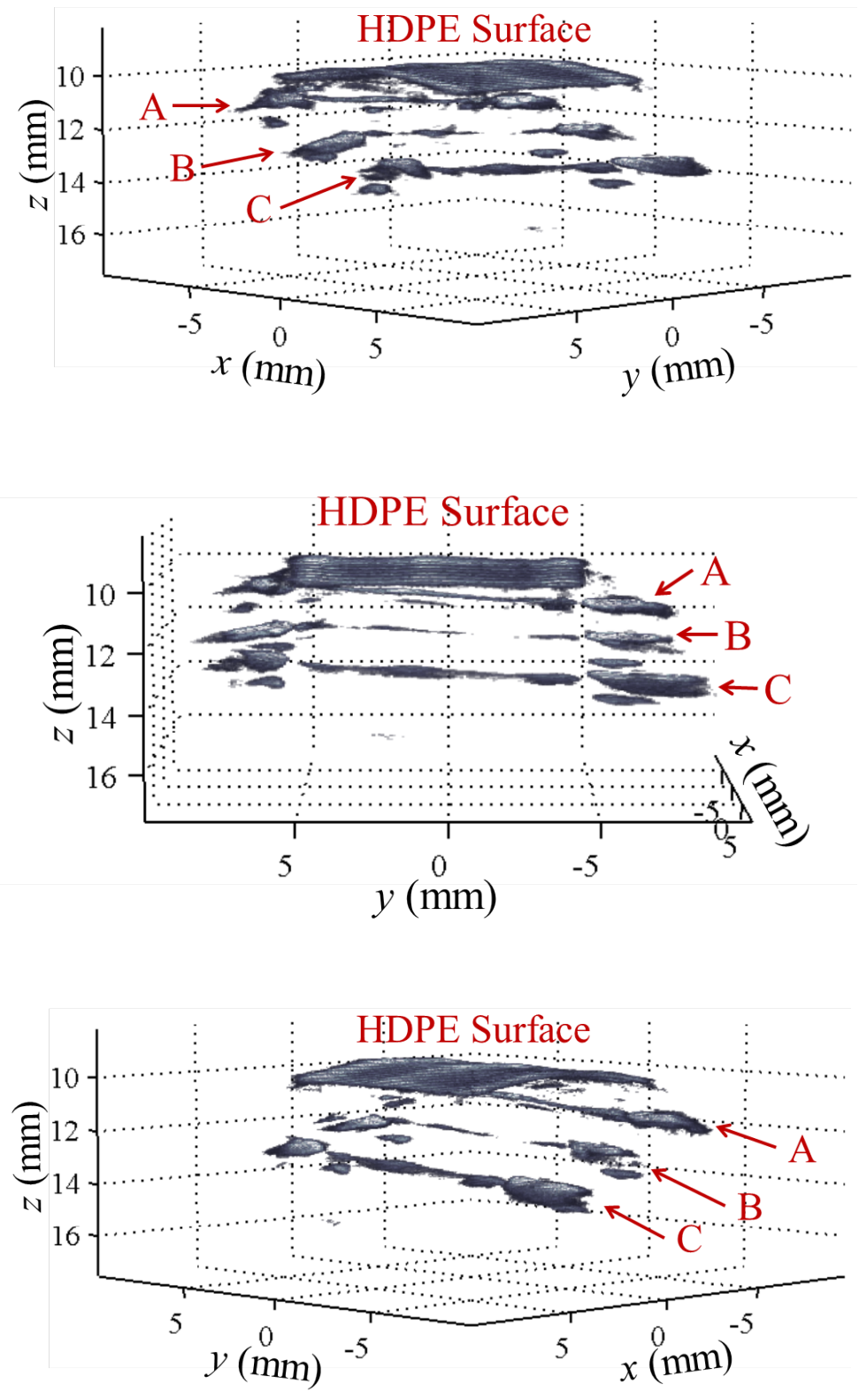

Figure 4.2.5: Volume rendering of $\mathrm{THz}$ SA tomographic data of HDPE test object obtained using SSF back-propagation shown at 3 different angles. Returns from the curved HDPE surface and wires A, B, and C are labeled. 

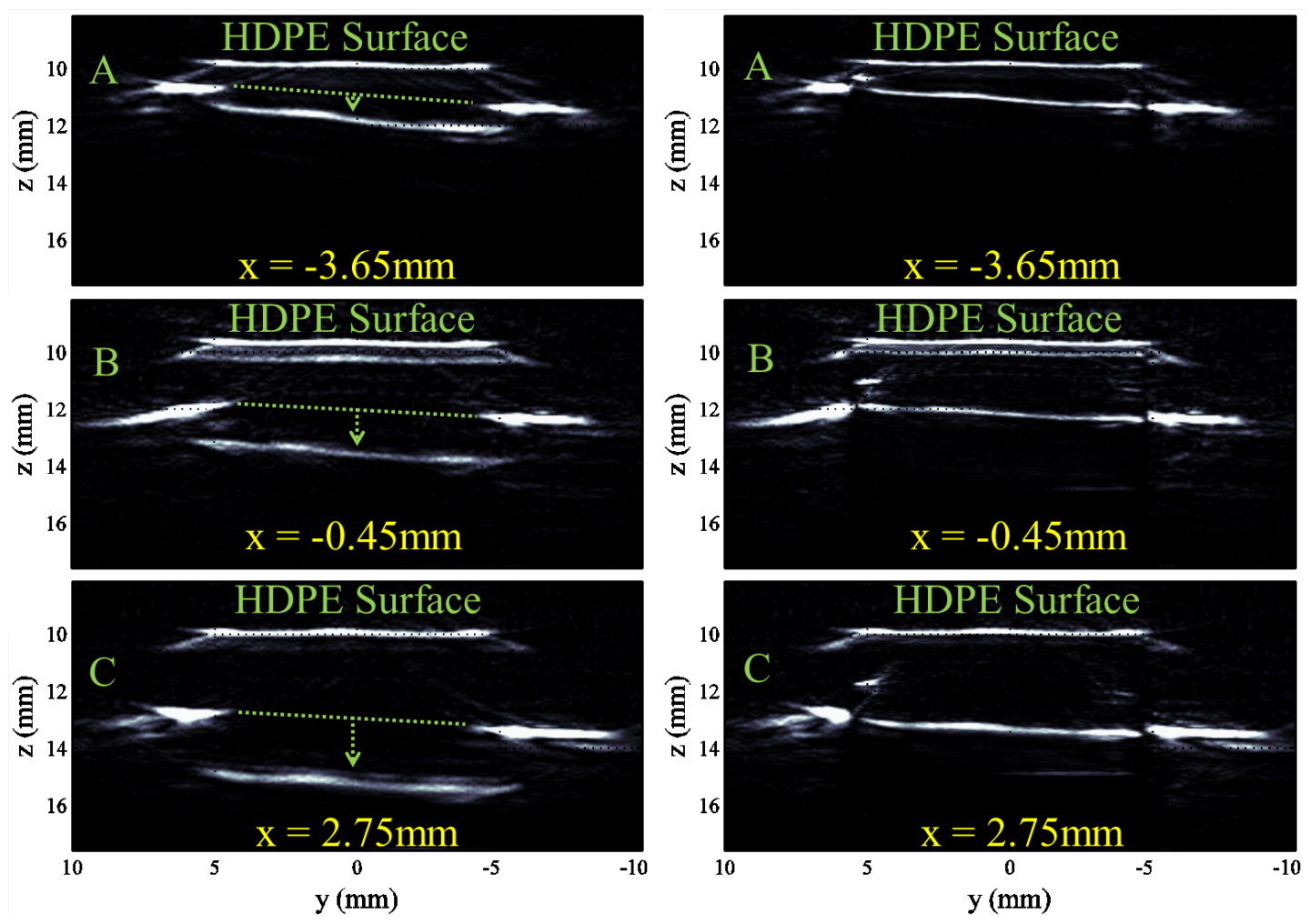

(a) Fourier Back-Propagation

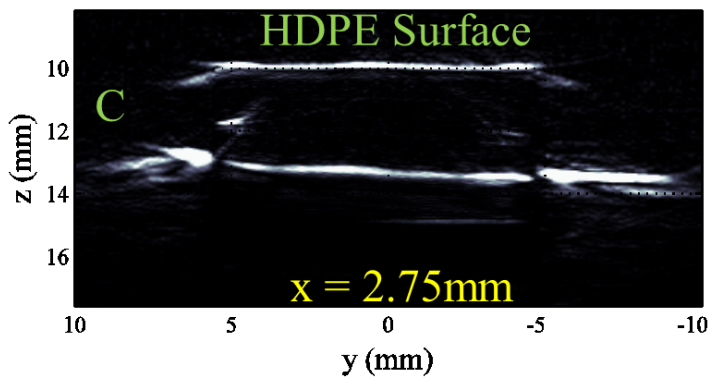

(b) SSF Back-Propagation

Figure 4.2.6: Side-by-side comparison of cross-sectional slices of THz SA tomographic data obtained using (a) FBP and (b) SSF back-propagation. Slices are shown at $x=-3.65 \mathrm{~mm},-0.45 \mathrm{~mm}$, and $2.75 \mathrm{~mm}$, corresponding to wires $\mathrm{A}, \mathrm{B}$, and $\mathrm{C}$, respectively. Returns from the HDPE surface are labeled and arrows indicate the downward shift of the internal wires from their correct location (dotted lines) due to refractive effects. The image distortions introduced by the HDPE have been largely corrected by the SSF method, as indicated by the continuity of the bright returns from the protruding ends of the wires and the segments of the wires within the HDPE. 
In order to demonstrate the ability of the SSF method to quantify the thickness of a curved dielectric layer, the hollow, hemicylindrical dielectric test object shown in Fig. 4.2.7 was fabricated using a 3D printer and smoothed in a room temperature acetone vapor bath. The object was designed with a $5 \mathrm{~mm}$ outer radius and an axiallysloped inner radius, ranging from $4 \mathrm{~mm}$ on one end to $3.5 \mathrm{~mm}$ on the other, as shown in Fig. 4.2.7a. The object therefore constitutes a smooth, curved, dielectric layer with axially-varying thickness. As shown in Fig. 4.2.7b and c, a piece of reflective copper tape was applied to the interior of the sample to provide a high-contrast return from the inner layer boundary. The ends of copper tape were intentionally left protruding from either end of the sample as shown in Fig. 4.2.7c to provide unrefracted points of reference for estimating the true location of the inner surface - the closest possible "ground truth" of the inner surface boundary's location in the experimental data.

The object was imaged using a Picometrix T-Ray 4000 THz time domain system, which was also used to carry out a separate transmission-mode measurement of a planar sample of the plastic extruded by the 3D printer, from which the material's $\mathrm{THz}$ refractive index was determined to be $n \approx 1.59$. As described in Sec. 4.1, the HDPE focusing lens with a $1^{\prime \prime}$ focal length was used in the T-Ray 4000's monostatic transceiver head to focus the $\mathrm{THz}$ beam into a point-like virtual transceiver at the focal point with a diverging $\mathrm{THz}$ beam below. This virtual transceiver was then raster scanned approximately $16 \mathrm{~mm}$ above the object's front surface to form a $300 \times$ 300-element planar synthetic aperture. While the autocorrelation of the $\mathrm{THz}$ pulses produced by the system have a full width at half maximum of $2.7 \mathrm{ps}$, corresponding to a maximum bandwidth of $0.37 \mathrm{THz}$, the aperture spacing in $x$ and $y$ was kept to $0.060 \mathrm{~mm}$ to satisfy $\lambda / 4$ spatial sampling up to $\approx 1.25 \mathrm{THz}$. 


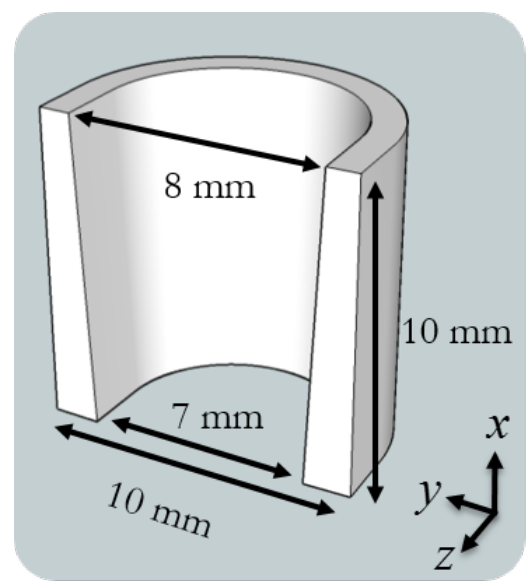

(a)

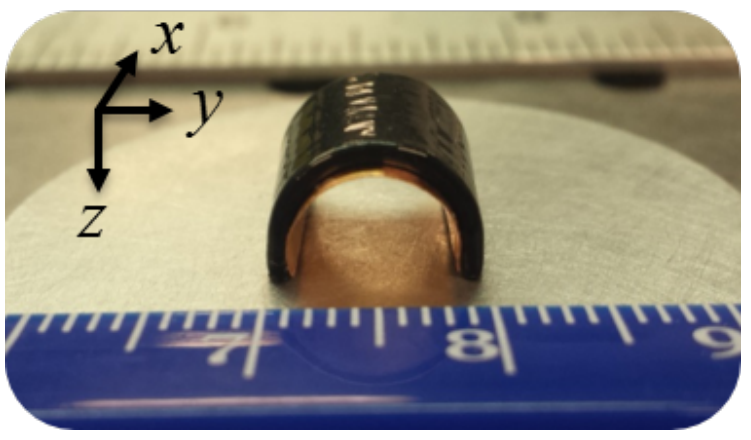

(b)

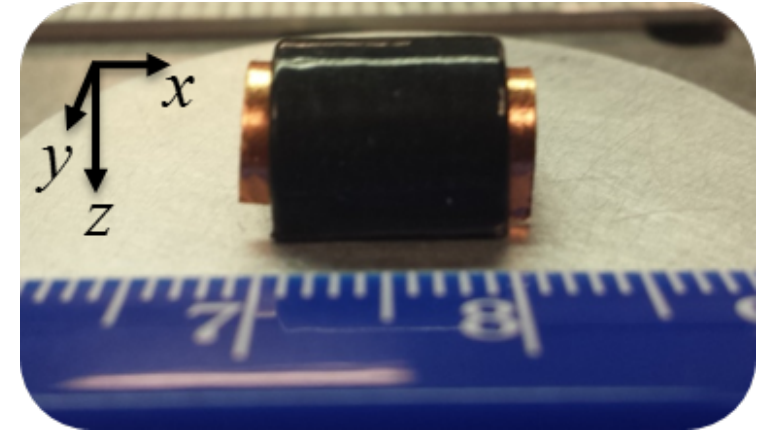

(c)

Figure 4.2.7: (a) Diagram of 3D printed hemicylindrical test object with $5 \mathrm{~mm}$ outer radius and axially-sloped inner radius, ranging from $4 \mathrm{~mm}$ on one end to $3.5 \mathrm{~mm}$ on the other. Copper tape was affixed to the inside of the sample to provide a highcontrast return as shown in the photographs (b) and (c). 

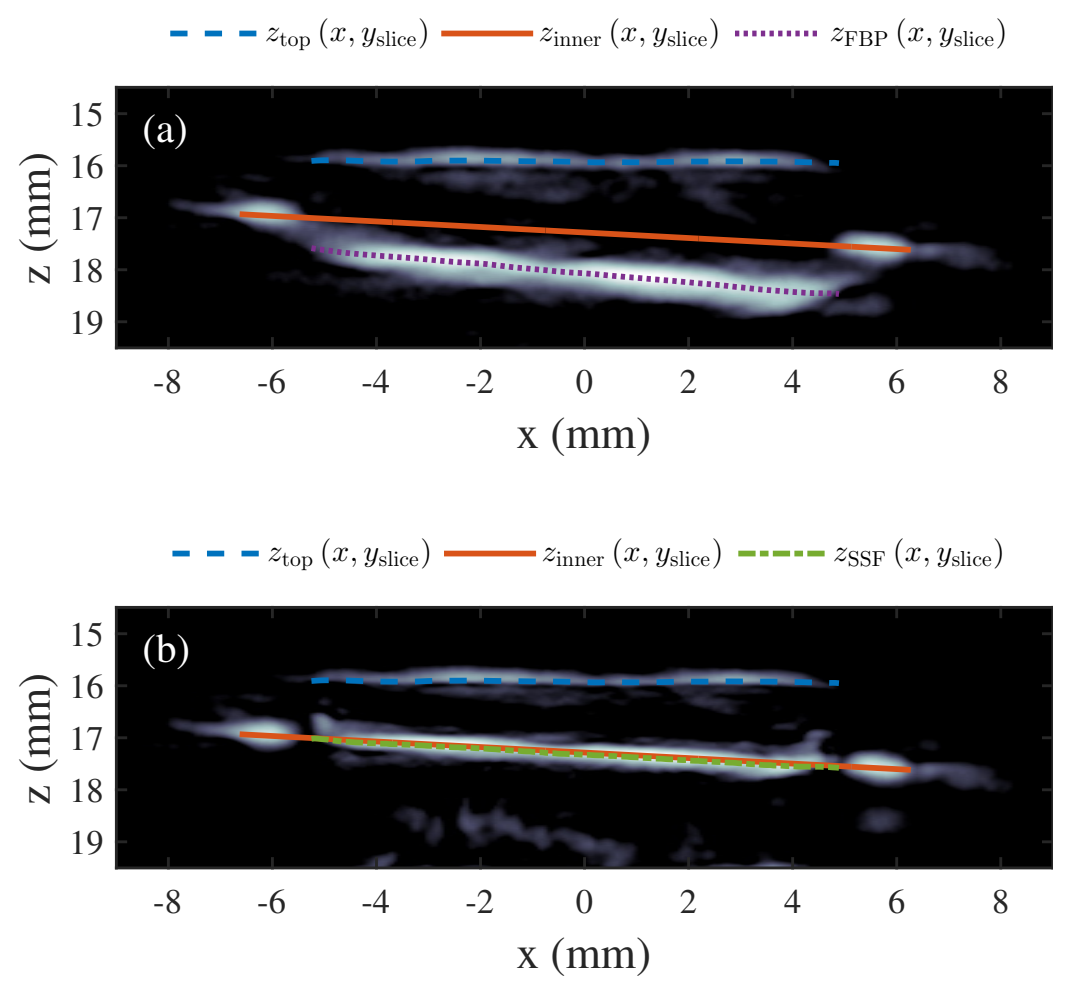

Figure 4.2.8: Cross-sectional slice images through 3D THz tomographic images produced using FBP (a) and SSF (b) methods at the same $y$ value. The top boundary $z_{\text {top }}\left(x, y_{\text {slice }}\right)$ and inner boundaries, estimated from the depth of the protruding ends $z_{\text {inner }}\left(x, y_{\text {slice }}\right)$ are visible in both images as dashed blue and solid orange lines, respectively. In the FBP-processed data (a), the inner surface $z_{\mathrm{FBP}}\left(x, y_{\text {slice }}\right)$ (dotted purple line) appears displaced vertically from $z_{\text {inner }}\left(x, y_{\text {slice }}\right)$ due to refraction. In the SSF-processed data (b), the refraction is corrected, bringing the inner surface $z_{\mathrm{SSF}}\left(x, y_{\text {slice }}\right)$ (dot-dashed green line) into alignment with $z_{\text {inner }}\left(x, y_{\text {slice }}\right)$.

The data were first processed using FBP as described in Sec. 3.2.1 with depth resolution $\Delta z=12.7 \mu \mathrm{m}$, corresponding to $1 / 8^{\text {th }}$ of the maximum step size in $(3.2 .36)$. Fig. 4.2.8a shows a cross-sectional slice through the resulting 3D image data along the axial $(x)$ direction, corresponding to the orientation in Fig. 4.2.7c. As the crosssectional images show, the locations of the upper surface boundary, inner boundary, and protruding copper tape ends are easily identifiable in the $3 \mathrm{D}$ data sets. This allows curves to be fit to the boundary locations in the image, resulting in a $2 \mathrm{D}$ surface 
profile for each interface. For the upper surface, the 2D profile is given by $z_{\text {top }}(x, y)$, a corresponding slice through which is shown as a dashed line in Fig. 4.2.8a. For the protruding copper tape ends, a linear fit was applied to connect the separate surface profiles to either side of the object, resulting in $z_{\text {inner }}(x, y)$, shown in Fig. 4.2.8a as a solid line. As mentioned previously, the protruding copper tape ends provide unrefracted points of reference that enable estimation of the copper tape's true location within the object. The profile $z_{\text {inner }}(x, y)$ is therefore treated as the "ground truth," i.e. the inner surface's true location. Applying the fitting process to the inner surface boundary in the FBP-processed data results in $z_{\mathrm{FBP}}(x, y)$, a slice through which is shown as the dotted line in Fig. 4.2.8a. The figure clearly shows that $z_{\mathrm{FBP}}(x, y)$ appears displaced vertically from $z_{\text {inner }}(x, y)$ due to refraction from the plastic layer, which is not corrected for by FBP processing.

Having localized the object's upper boundary $z_{\text {top }}(x, y)$ using the FBP-processed 3D image, the data were reprocessed using the SSF method, as described in Sec. 3.2.2, assuming the experimentally-determined refractive index of $n=1.59$ for the region below the upper surface and $n=1$ elsewhere in the image space. Fig. 4.2.8b shows a cross-sectional slice through the resulting SSF-processed data at the same location as Fig. 4.2.8a. The slices through $z_{\text {top }}(x, y)$ and $z_{\text {inner }}(x, y)$ are overlaid for reference. As Fig. 4.2.8b shows, the inner surface boundary in the SSF-processed data is much closer to the ground truth $z_{\text {inner }}(x, y)$ than that in the FBP processed data shown in Fig. 4.2.8a. This is due to the SSF method's correcting for refraction within the dielectric object. Applying the fitting process to the inner surface boundary in the SSF-processed data results in $z_{\mathrm{SSF}}(x, y)$, a slice through which is shown as the dotdashed line in Fig. 4.2.8b. With the exception of some edge effects at $y \approx \pm 5 \mathrm{~mm}$, the SSF processing brings the apparent depth $z_{\mathrm{SSF}}(x, y)$ of the inner boundary into 
much closer alignment with the ground truth $z_{\text {inner }}(x, y)$ from the protruding ends.

The thickness of the curved dielectric layer can be obtained from the vertical distance between the object boundaries in the image space. This can be done using the ground truth from the protruding tape ends, resulting in

$$
d_{\text {true }}(x, y)=z_{\text {inner }}(x, y)-z_{\text {top }}(x, y)
$$

and the apparent locations of the inner boundaries in the FBP and SSF-processed data, i.e.

$$
\begin{aligned}
& d_{\mathrm{FBP}}(x, y)=z_{\mathrm{FBP}}(x, y)-z_{\mathrm{top}}(x, y), \\
& d_{\mathrm{SSF}}(x, y)=z_{\mathrm{SSF}}(x, y)-z_{\mathrm{top}}(x, y) .
\end{aligned}
$$

Fig. 4.2.9 shows these thicknesses calculated for the cross-sectional image slices shown in Fig. 4.2.8. The figure shows $d_{\mathrm{SSF}}\left(x, y_{\text {slice }}\right)$ is in much greater agreement with the ground truth $d_{\text {true }}\left(x, y_{\text {slice }}\right)$ than $d_{\mathrm{FBP}}\left(x, y_{\text {slice }}\right)$, as would be expected given the improvement in accuracy provided by SSF processing.

Defining the thickness error in the FBP and SSF-processed data as

$$
\begin{aligned}
\Delta d_{\mathrm{FBP}}(x, y) & =d_{\mathrm{FBP}}(x, y)-d_{\text {true }}(x, y) \\
& =z_{\mathrm{FBP}}(x, y)-z_{\text {inner }}(x, y)
\end{aligned}
$$




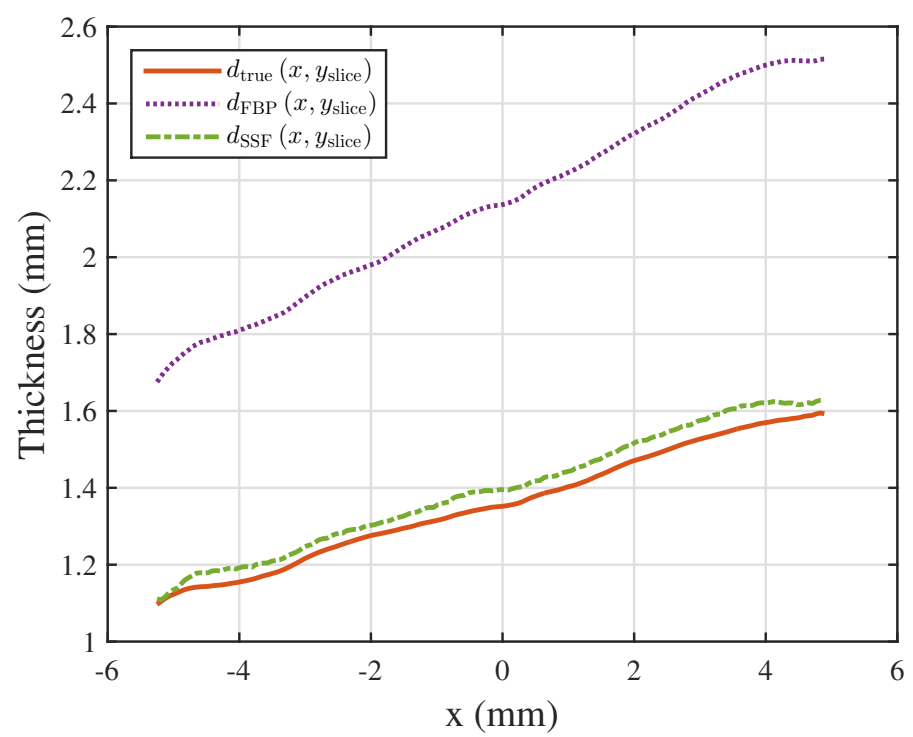

Figure 4.2.9: Layer thickness estimates calculated using (4.2.1)-(4.2.3) for the image data in Fig. 4.2.8.

and

$$
\begin{aligned}
\Delta d_{\mathrm{SSF}}(x, y) & =d_{\mathrm{SSF}}(x, y)-d_{\text {true }}(x, y) \\
& =z_{\mathrm{SSF}}(x, y)-z_{\text {inner }}(x, y),
\end{aligned}
$$

respectively, allows quantifying the thickness error over a range of $y$ values centered about the apex of the object's cylindrically-curved upper surface, as shown in Fig. 4.2.10. Fig. 4.2.10a shows a general increase in $\Delta d_{\mathrm{FBP}}(x, y)$ with increasing $x$ and decreasing $y$ (i.e. in the direction of the upper right corner of the image). The increasing error along the $x$ direction is due to refractive effects causing the apparent axial slope of the inner surface boundary to be steeper than that of the ground truth, as shown in Figs. 4.2.8a and 4.2.9. As Fig. 4.2.8b shows, SSF back-propagation corrects for this refraction, bringing $z_{\text {inner }}(x, y)$ and $z_{\mathrm{SSF}}(x, y)$ into closer alignment, reducing the discrepancy in the axial slope, which in turn reduces the $x$-varying 

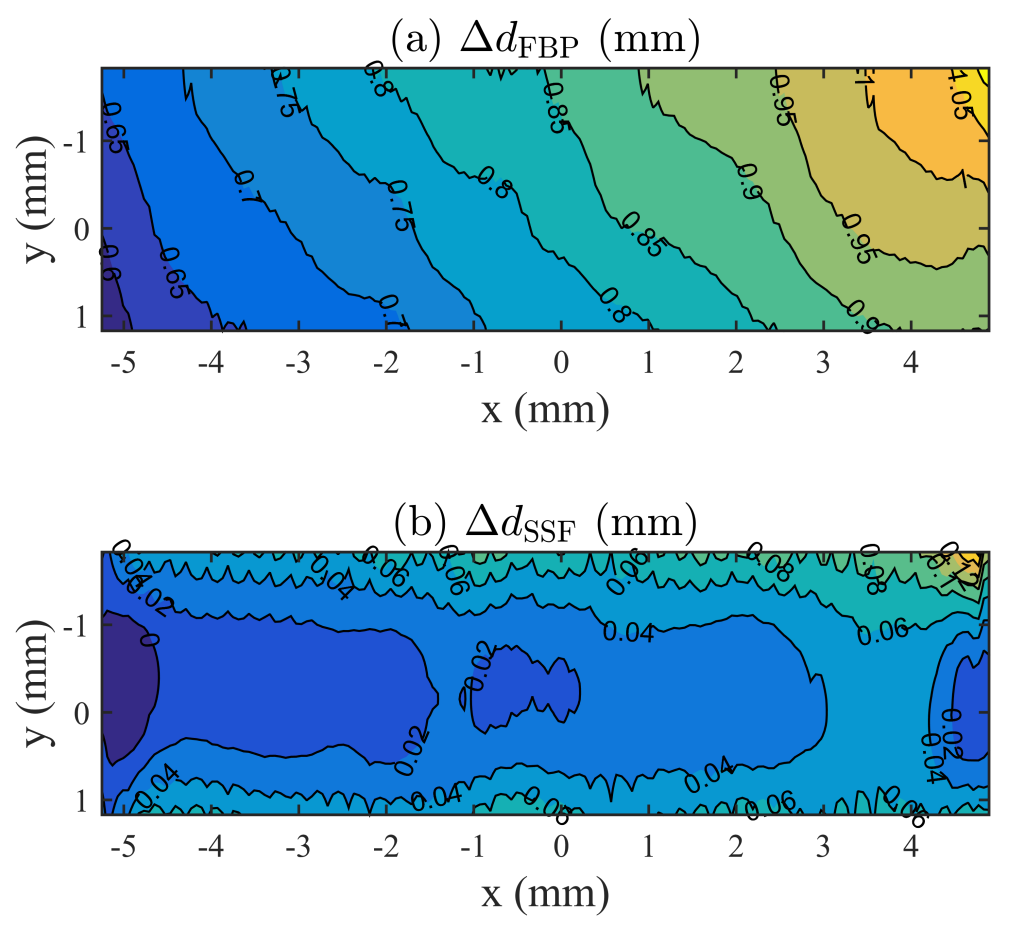

Figure 4.2.10: Contour plots of layer thickness error in millimeters. (a) Layer thickness error from FBP-processed data calculated using (4.2.4). (b) Layer thickness error from SSF-processed data calculated using (4.2.5). While some error remains, SSF processing significantly reduces thickness error. 
change in the thickness error from that shown in Fig. 4.2.10a to that in Fig. 4.2.10b.

The increase in $\Delta d_{\mathrm{FBP}}(x, y)$ in the $-y$ direction in Fig. 4.2.10a is due to the fact that the 3D-printed sample's outer and inner surface boundaries are not exactly coaxial - i.e. the centers of curvature of $z_{\text {top }}(x, y)$ and $z_{\text {inner }}\left(x, y_{\text {slice }}\right)$ are located at slightly different $y$ values. This axial asymmetry combined with the refractive effects results in $z_{\mathrm{FBP}}(x, y)$ having a center of curvature at $y \approx-0.20 \mathrm{~mm}$ compared to $y \approx$ $-0.30 \mathrm{~mm}$ for $z_{\text {inner }}(x, y)$. This $\approx 0.1 \mathrm{~mm}$ misalignment of the centers of curvature then maps into (4.2.4) and gives rise to the asymmetry along the $y$ dimension shown in Fig. 4.2.10a. In contrast, SSF processing largely mitigates these refractive effects, resulting in $z_{\mathrm{SSF}}(x, y)$ having a center of curvature at $y \approx-0.29 \mathrm{~mm}$. With the misalignment of the centers of curvature reduced to $\approx 0.01 \mathrm{~mm}$, the asymmetry in Fig. 4.2.10b is decreased significantly from that in Fig. 4.2.10a.

The overall increase in accuracy due to SSF processing is also evident in the mean of the thickness error, \pm one standard deviation, which is approximately $0.0362 \pm 0.0217 \mathrm{~m}$ $\mathrm{m}$ for $\Delta d_{\mathrm{SSF}}(x, y)$, compared to $0.817 \pm 0.112 \mathrm{~mm}$ for $\Delta d_{\mathrm{FBP}}(x, y)$. The remaining error due to approximations made in the derivation of the SSF-operator will be discussed in greater detail in the following section.

\subsection{Error Analysis Simulations}

As mentioned in Sec. 3.2.2, the SSF method is based on approximations that are valid for small lateral changes in the refractive index and small lateral propagation angles. This section relates the analytic expression (3.2.39) for the SSF propagator error, presented in Sec. 3.2.2, to the experimental results presented in Sec. 4.2.3. 
Idealized simulations are then shown to further examine the influence of propagator error on the SSF method's ability to correctly localize the boundaries and estimate the thickness of a dielectric layer in an object under test. These results are featured in a manuscript recently submitted for publication in the IEEE Transactions on THz Science and Technology [41].

From Fig. 3.2.3, the propagation angle $\theta_{p}$ is equal to the tilt angle of a curved surface's tangent plane. Given the profile $z_{\text {top }}(x, y)$ of the cylindrically-curved upper surface of the 3D printed test object described in Sec. 4.2.3, the surface's tangent plane tilt angle was calculated over the extent of the surface using

$$
\theta_{p}(x, y)=\tan ^{-1}\left[\frac{\partial}{\partial y} z_{\text {top }}(x, y)\right]
$$

which was found to be approximately bounded by $\left|\theta_{p}\right| \leq 32^{\circ}$. This range of propagation angles combined with the refractive indices of the plastic $(n \approx 1.59)$ and surrounding air $(n=1)$ allows calculation of the propagator error using (3.2.39). Fig. 4.3.1 shows lines corresponding to the resulting error in the data overlaid on the relative propagator error shown in Fig. 3.2.4. These lines indicate the relative propagator error in the experimental data stays within approximately $\pm 0.05( \pm 5 \%)$.

While this analysis puts the experimental data in context with respect to the error described in Sec. 3.2.2, the cylindrically-curved surface presents a $y$-varying change in the tangent plane tilt angle, as shown in Fig. 3.2.3, and therefore a laterally-varying change in $\theta_{p}$. In order to quantify the effect of propagation angle-induced error on the layer thickness estimate, the simulation test scenario, shown in Fig. 4.3.2 was devised. The test case consists of a $1 \mathrm{~mm}$-thick tilted planar dielectric layer with top 


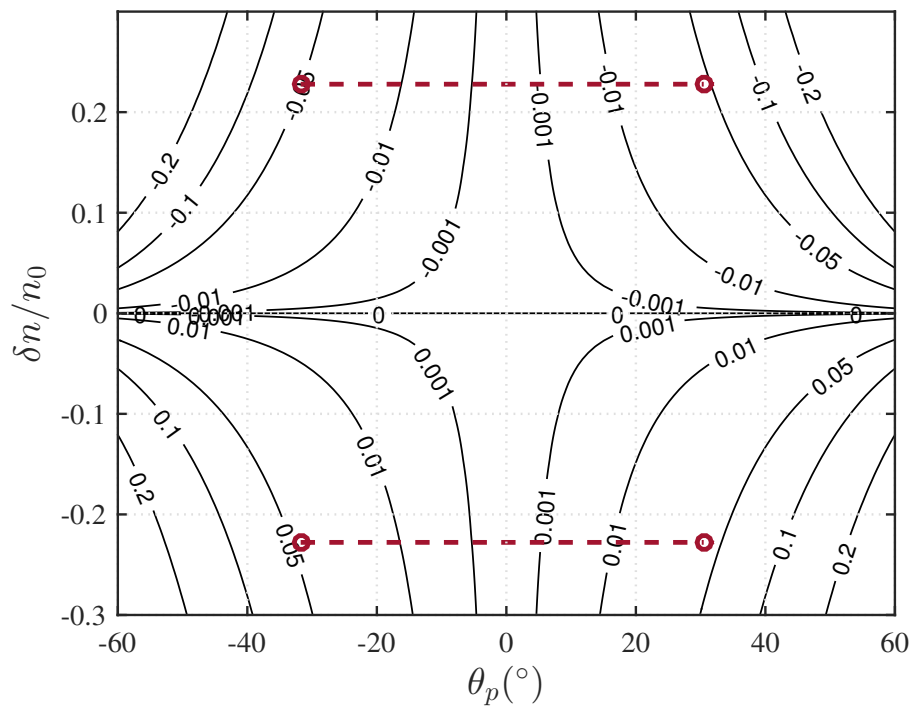

Figure 4.3.1: Relative error in the SSF propagator (3.2.39) as a function of propagation angle $\theta_{p}$ and relative refractive index perturbation $\delta n / n_{0}$ along with lines representing where the error in the data set presented in Sec. 4.2 .3 falls.

and bottom boundaries at

$$
z_{\text {top }}\left(x, \theta_{p}\right)=z_{1}-x \tan \theta_{p}
$$

and

$$
z_{\text {bottom }}\left(x, \theta_{p}\right)=z_{2}-x \tan \theta_{p},
$$

where $z_{1}=15 \mathrm{~mm}$ and $z_{2}=16 \mathrm{~mm}$ as shown in Fig. 4.3.2. The fields on the array reflected from the front and back surfaces of the tilted layer (ignoring etalon reflections reverberating within the layer for simplicity) are given by

$$
\begin{aligned}
\psi_{r}\left(x, \theta_{p}\right)= & R_{1} e^{-i 2 k r\left(x, \theta_{p}\right)} \\
& +R_{2} T_{1} T_{2} e^{-i 2 k\left(r\left(x, \theta_{p}\right)+n \Delta r\left(\theta_{p}\right)\right)},
\end{aligned}
$$




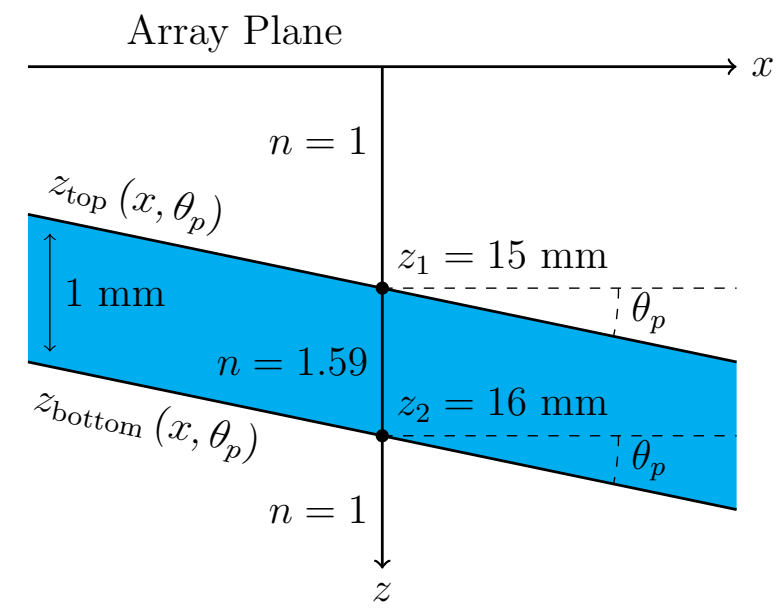

Figure 4.3.2: Diagram of tilted dielectric layer assumed in simulations.

where

$$
\begin{aligned}
& R_{1}=\frac{1-n}{1+n}, \\
& R_{2}=\frac{n-1}{1+n},
\end{aligned}
$$

and

$$
T_{1,2}=1+R_{1,2}
$$

are the Fresnel reflection and transmission coefficients, respectively, at normal incidence for the upper and lower boundaries. From the geometry in Fig. 4.3.2,

$$
r\left(x, \theta_{p}\right)=z_{1} \cos \theta_{p}-x \sin \theta_{p}
$$

and

$$
\Delta r\left(\theta_{p}\right)=\left(z_{2}-z_{1}\right) \cos \theta_{p}
$$

Reflected fields on the array were calculated using (4.3.4) assuming spectrally-flat 
incident pulses with a bandwidth of $1.25 \mathrm{THz}$, corresponding to the $\lambda / 4$ limit for $0.060 \mathrm{~mm}$ array spacing used in the experimental data described in Sec. 4.2.3. The simulation data were then processed with both FBP and SSF back-propagation using a propagation step size $\Delta z=12.7 \mu \mathrm{m}$ as in the measured data from Sec. 4.2.3.

Fig. 4.3.3 shows the images resulting from $\theta_{p}=32^{\circ}$, the 'worst-case' relative propagation error in the experimental data along with lines corresponding to the upper surface $z_{\text {top }}\left(x, \theta_{p}=32^{\circ}\right)$ and lower surface $z_{\text {bottom }}\left(x, \theta_{p}=32^{\circ}\right)$. Just as in the experimental results in Fig. 4.2.8, the lower surface boundary appears lower than $z_{\text {bottom }}\left(x, \theta_{p}=32^{\circ}\right)$ in the FBP-processed image (Fig. 4.3.3a) due to refraction, which is largely corrected for by SSF processing, as shown in Fig. 4.3.3b. However, Fig. 4.3.3b also illustrates the effect of propagator error, which gives rise to a laterally-varying difference in the correct and apparent boundary locations in the image. This lateral increase in boundary location mismatch is due to the fact that propagator error accumulates in regions in which the refractive index varies laterally. In the case of a tilted planar dielectric layer, error begins accumulating when the propagation step $z+\Delta z$ reaches the upper-left edge of $z_{\text {top }}\left(x, \theta_{p}\right)$ in Fig. 4.3.2. As the fields are backpropagated through the layer, the error accumulates, resulting in an increase in total error with increasing $z$. The apparent boundary locations in the resulting image (Fig. 4.3.3b) are therefore increasingly displaced from $z_{\text {top }}\left(x, \theta_{p}\right)$ and $z_{\text {bottom }}\left(x, \theta_{p}\right)$ with increasing $z$. While the accuracy of the boundary localization suffers slightly from the SSF propagator error, the estimated location of the lower surface is still much more accurate than for FBP.

Applying linear fits to the apparent locations of the upper and lower boundaries in the 2D image space allows calculation of the apparent layer thicknesses in a similar fashion 

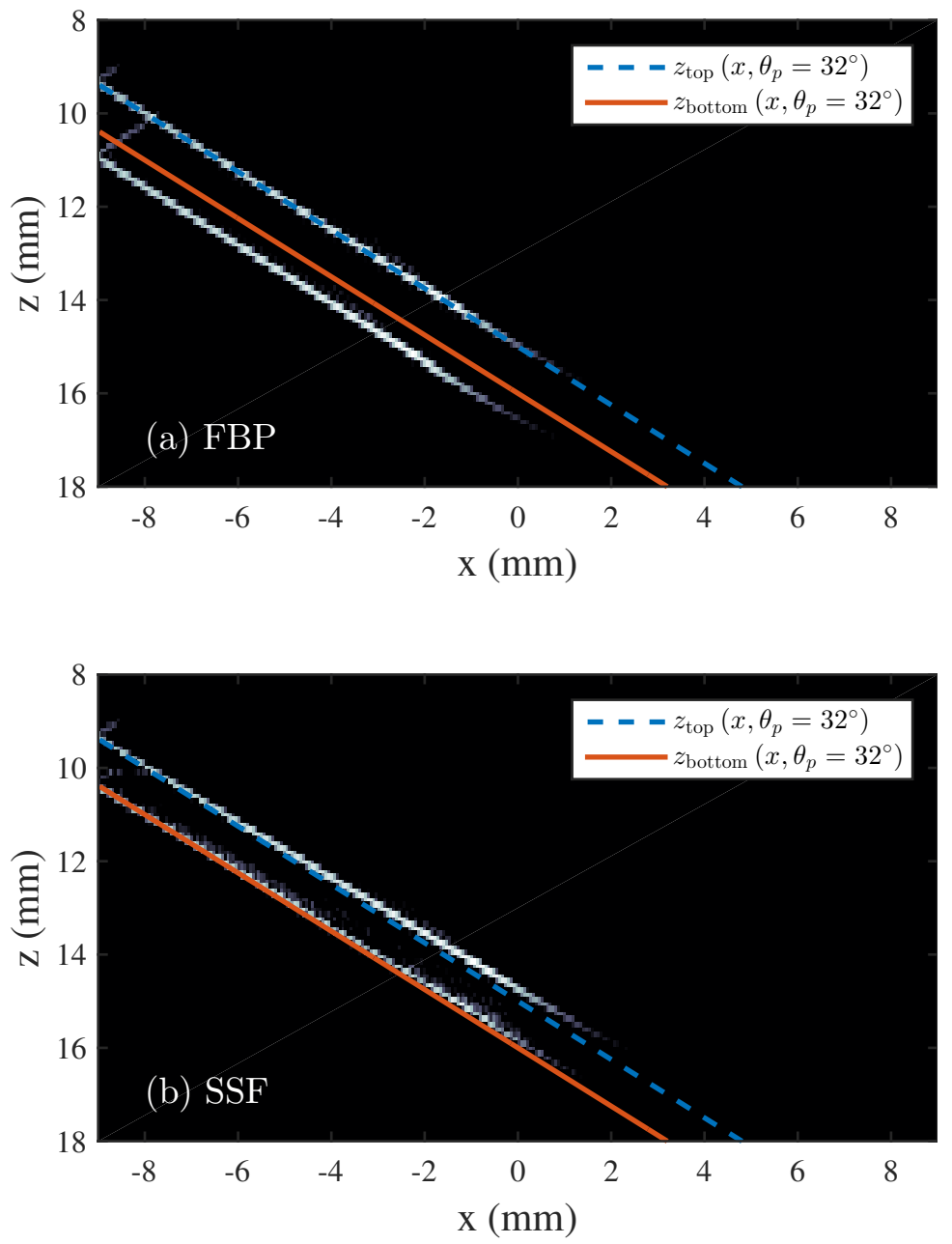

Figure 4.3.3: Imaging results from simulated data from tilted dielectric slab shown in Fig. 4.3.2 for the case of $\theta_{p}=32^{\circ}$, shown with $z_{\text {top }}\left(x, \theta_{p}\right)$ and $z_{\text {bottom }}\left(x, \theta_{p}\right)$ for reference. (a) Results of FBP processing, which doesn't correct for refraction within the object. (b) Results of SSF processing given the layer's THz refractive index $n=1.59$. 


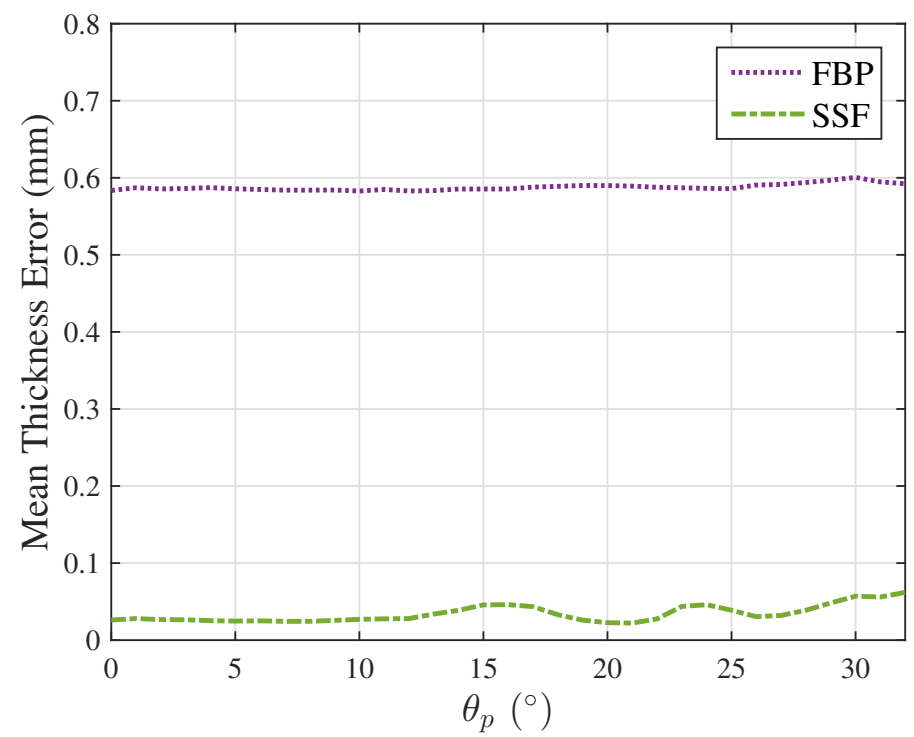

Figure 4.3.4: Thickness error for a range of tilt angles for both FBP and SSF processed data.

to that described for the experimental data in Sec. 4.2.3. The resulting estimates of the boundary locations are given by $\hat{z}_{\text {top }}^{\mathrm{FBP}}\left(x, \theta_{p}\right), \hat{z}_{\mathrm{bottom}}^{\mathrm{FBP}}\left(x, \theta_{p}\right), \hat{z}_{\mathrm{top}}^{\mathrm{SSF}}\left(x, \theta_{p}\right)$, and $\hat{z}_{\text {bottom }}^{\mathrm{SSF}}\left(x, \theta_{p}\right)$, where the superscript indicates the processing applied to the data from which the estimates originate. This allows estimating the thickness errors in the simulated data using

$$
\Delta d_{\mathrm{FBP}}\left(x, \theta_{p}\right)=\hat{z}_{\mathrm{bottom}}^{\mathrm{FBP}}\left(x, \theta_{p}\right)-\hat{z}_{\text {top }}^{\mathrm{FBP}}\left(x, \theta_{p}\right)-d_{\text {true }}
$$

and

$$
\Delta d_{\mathrm{SSF}}\left(x, \theta_{p}\right)=\hat{z}_{\mathrm{bottom}}^{\mathrm{SSF}}\left(x, \theta_{p}\right)-\hat{z}_{\mathrm{top}}^{\mathrm{SSF}}\left(x, \theta_{p}\right)-d_{\mathrm{true}}
$$

where $d_{\text {true }}=z_{2}-z_{1}=1 \mathrm{~mm}$ is the true thickness of the dielectric layer. Fig. 4.3 .4 shows plots of the mean thickness error, calculated by averaging (4.3.10) and (4.3.11) over $x$, for $\theta_{p}$ ranging from $0^{\circ}$ to $32^{\circ}$. In both the FBP and SSF results in Fig. 4.3.4, 
the image pixel size $(\Delta x=60 \mu \mathrm{m} \times \Delta z=12.7 \mu \mathrm{m})$ introduces artifacts in the linear fit routine used to localize the boundaries, which give rise to small deviations in the thickness error. In spite of these small deviations however, two main conclusions can be drawn from the results shown in Fig. 4.3.4. First and foremost, the performance of SSF decreases with increasing $\theta_{p}$, as would be expected from the analysis in Sec. 3.2.2. Beginning around the $\theta_{p} \approx 12^{\circ}$ mark, propagator error combined with the aforementioned artifacts in the linear fit routine act to increase the thickness error, which reaches local maxima at $\theta_{p} \approx 16^{\circ}$ and $24^{\circ}$, before increasing monotonically with increasing $\theta_{p}$. Second, even though the performance of SSF processing suffers with increasing $\theta_{p}$, the thickness error remains much lower than that from FBP processing. 


\section{Chapter 5}

\section{Conclusions and Future Work}

The ability of $\mathrm{THz}$ waves to penetrate many non-polar dielectric materials combined with their sub-millimeter wavelengths and the coherent, broadband nature of many THz systems makes the technology uniquely suited for many nondestructive evaluation applications. However, conventional THz imaging methods such as TPI are not easily adapted for generating 3D images of objects with curved interfaces. Terahertz SA tomography offers several advantages over more conventional TPI methods, including a simplified scan geometry and dynamic numerical focusing to multiple depths with a single data set. However, the irregular surface topographies of some objects of interest complicate numerical focusing, resulting in refractive effects that distort the tomographic images of the object's interior.

This work addresses this issue by introducing parabolic equation (PE) methods namely, the split-step Fourier method - as a means of correcting for the refraction introduced by such curved boundaries. As such, this work represents a novel combination of THz technology, phased array imaging, reflection-mode diffraction tomography, and PE methods. The last of these subjects - PE methods - are a family of wave-equation based propagation techniques commonly used to model electromagnet- 
ic and acoustic wave propagation through inhomogeneous media and/or environments by researchers in the underwater acoustics, seismology, and atmospheric electromagnetic propagation communities. Despite its nearly 70-year history [104], PE methods have only relatively recently been applied to signal processing for applications in ground-penetrating radar [35, 36] and medical ultrasound [37, 38]. To the author's knowledge, this work [39-41] is the first time PE methods have been applied to the $\mathrm{THz}$ frequency band.

\subsection{Broader Impacts and Future Work}

This dissertation advances the capabilities of $\mathrm{THz} 3 \mathrm{D}$ imaging technology by developing an efficient, physics-based image processing method capable of correcting for refractive effects introduced in $3 \mathrm{D} \mathrm{THz}$ tomographic images of transparent objects with irregular boundaries. Though the original motivation for this work was to allow focusing to within the curved layers of pharmaceutical tablets in order to non-destructively estimate the thickness of the various enteric coating layers, the method developed is much more broadly useful and is applicable to a far larger set of THz imaging scenarios in which accurate 3D images of a transparent object's internal structure are sought. Other applications of this method could include NDE and watermarking of IC chips or diagnostic imaging of dental caries.

The specific PE method used in this work - the split-step Fourier method - was selected due to its computational efficiency and conceptual simplicity. However, many other PE methods in common use today may also be applicable to 3D $\mathrm{THz}$ tomographic imaging problems. One logical next step of this work would be applying the split-step Padé parabolic equation method [110, 111] developed by Michael Collins. 
This method is used exclusively by the underwater acoustics community and the U.S. Navy due to its combination of accuracy and computational efficiency $[112,143,144]$. Though typically applied in 2D acoustic simulations, more recent work has extended it to the 3D case [115]. The material presented in this dissertation represents the first step in this direction, laying the foundation for further work in bringing the powerful family of PE methods to bear on THz phased-array tomographic imaging problems.

\subsection{Publications}

Below is a list of publications in which the author of this dissertation proposal either authored or co-authored during the course of conducting this research.

- G. P. Kniffin and L. M. Zurk, "Parabolic Equation Methods for Terahertz 3D Synthetic Aperture Imaging," submitted for publication in the IEEE Transactions on Terahertz Science and Technology, March 18, 2016.

- G. P. Kniffin and L. M. Zurk, "Terahertz Non-Destructive Sensing of Layered Materials with Curved Surfaces," Proceedings of the 16th International Radar Symposium (IRS), 2015.

- S. Schecklman, L. M. Zurk, and G. Kniffin, "Terahertz Scattering from Contaminants Embedded in Textile Rope and Sling Materials," IEEE Symposium on Antennas and Propagation and North America Science Meeting, Session: Subsurface Remote Sensing, 2015

- S. Schecklman, G. Kniffin, and L. M. Zurk, "Terahertz Non-destructive Evaluation of Layered Media with the Maximum Likelihood Estimator," Proceed- 
ings of the International Symposium on Optomechatronic Technologies (ISOT), 2014.

- S. Schecklman, G. Kniffin, and L. M. Zurk, "Terahertz Non-destructive Evaluation of Ropes and Slings," Proceedings of the International Symposium on Optomechatronic Technologies (ISOT), 2014.

- G. Kniffin, L. M. Zurk, S. Schecklman, S. C. Henry, "3D Terahertz Synthetic Aperture Imaging of Objects with Arbitrary Boundaries," Proceedings of SPIE - The International Society for Optical Engineering, 2013.

- S.C. Henry, G. Kniffin, L.M. Zurk, "3-D Broadband Terahertz Synthetic Aperture Imaging," IRMMW-THz, Sept, 2012.

- G. P. Kniffin, L. M. Zurk, "Model-Based Material Parameter Estimation for Terahertz Reflection Spectroscopy," IEEE Transactions on Terahertz Science and Technology, Vol. 2, No. 2, pp. 231-241, March 2012.

- L.M. Zurk, S.C. Henry, G. P. Kniffin, "Terahertz material detection from diffuse surface scattering," Journal of Applied Physics, Vol. 109, No. 9, May 1, 2011.

- S. Schecklman, L. M. Zurk, S. Henry, G. Kniffin, D. Duncan, "Terahertz reflection spectroscopy for random rough surfaces," Journal of Applied Physics, Vol. 109, No. 9, May 1, 2011.

- G. Kniffin, S. Schecklman, J. Chen, L. M. Zurk, B. Pejcinovic, A. Timchenko, "Measurement and Modeling of THz Spectral Signatures from Layered Material," Proceedings of SPIE - The International Society for Optical Engineering, 2010 . 
- S.C. Henry, G. Kniffin, S. Schecklman, and L. M. Zurk, "Measurement and Modeling of Rough Surface Effects on Terahertz Spectroscopy," Proceedings of SPIE - The International Society for Optical Engineering, 2010. 


\section{Bibliography}

[1] J. A. Zeitler, Y. Shen, C. Baker, P. F. Taday, M. Pepper, and T. Rades, "Analysis of coating structures and interfaces in solid oral dosage forms by three dimensional terahertz pulsed imaging," Journal of Pharmaceutical Sciences, vol. 96, no. 2, pp. 330-340, 2007.

[2] M. Kemp, P. Taday, B. Cole, J. Cluff, A. Fitzgerald, and W. Tribe, "Security applications of terahertz technology," English, Proceedings of SPIE - The International Society for Optical Engineering, vol. 5070, pp. 44 -52, 2003.

[3] J. Chen, Y. Chen, H. Zhao, G. J. Bastiaans, and X.-C. Zhang, "Absorption coefficients of selected explosives and related compounds in the range of 0.1-2.8 thz," English, Optics Express, vol. 15, no. 19, pp. 12060 -12 067, 2007.

[4] S. Zhong, Y.-C. Shen, L. Ho, R. May, J. Zeitler, M. Evans, P. Taday, M. Pepper, T. Rades, K. Gordon, R. Muller, and P. Kleinebudde, "Non-destructive quantification of pharmaceutical tablet coatings using terahertz pulsed imaging and optical coherence tomography," English, Optics and Lasers in Engineering, vol. 49, no. 3, pp. $361-5,2011$.

[5] R. May, M. Evans, S. Zhong, C. Byers, L. Gladden, Y. Shen, and J. Zeitler, "Terahertz pulsed imaging of surface variations on pharmaceutical tablets," English, Piscataway, NJ, USA, 2010, pp. 1-2. 
[6] T. Kurabayashi, S. Yodokawa, and S. Kosaka, "Terahertz imaging through paint layers," in 2012 37th International Conference on Infrared, Millimeter, and Terahertz Waves, 2012.

[7] T. Kurabayashi, S. Sakai, and K. Fujino, "Sub-terahertz imaging of a painted steel," in Infrared Millimeter and Terahertz Waves (IRMMW-THz), 2010 35th International Conference on, IEEE, 2010, pp. 1-2.

[8] S Wietzke, N Krumbholz, C Jördens, B Baudrit, M Bastian, and M Koch, "Inspection of plastic weld joints with terahertz imaging," in Optical Metrology, International Society for Optics and Photonics, 2007, 66163N-66163N.

[9] S Wietzke, C Jansen, C Jördens, N Krumbholz, N Vieweg, M Scheller, M. Shakfa, D Romeike, T Hochrein, M Mikulics, et al., "Industrial applications of thz systems," in International Symposium on Photoelectronic Detection and Imaging 2009, International Society for Optics and Photonics, 2009, pp. $738506-738506$.

[10] S Wietzke, C Jansen, N Krumbholz, O Peters, N Vieweg, C Jördens, M Scheller, D Romeike, T Jung, M Reuter, et al., "Terahertz spectroscopy: a powerful tool for the characterization of plastic materials," in Solid Dielectrics (ICSD), 2010 10th IEEE International Conference on, IEEE, 2010, pp. 1-4.

[11] M. Yamashita, T. Kiwa, M. Tonouchi, and K. Kawase, "Development of laserterahertz emission microscope for inspecting the electrical faults in semiconductor devices," in Conference on Lasers and Electro-Optics, Optical Society of America, 2004, CThN5.

[12] M. Yamashita, C. Otani, K. Kawase, T. Matsumoto, K. Nikawa, S. Kim, H. Murakami, and M. Tonouchi, "Backside observation of large-scale integrat- 
ed circuits with multilayered interconnections using laser terahertz emission microscope," Applied Physics Letters, vol. 94, no. 19, p. 191 104, 2009.

[13] M Yamashita, C Otani, T Matsumoto, Y Midoh, K Miura, K Nikawa, K Nakamae, and M Tonouchi, "Non-bias inspection of electrical failures in lsi interconnects using ltem prototype system," in Infrared Millimeter and Terahertz Waves (IRMMW-THz), 2010 35th International Conference on, IEEE, 2010, pp. $1-2$.

[14] M Vandewal, J Depauw, K Rombaut, R Beigang, J Jonuscheit, P Mounaix, I. S. de Ocáriz, R. M. Edo, A Priegue, and Y Sternberg, "Development and optimization of thz ndt on aeronautics composite multilayered structures," in SPIE Defense, Security, and Sensing, International Society for Optics and Photonics, 2012, 83630Y-83630Y.

[15] M. Bohn, C. Stoik, and J. Blackshire, "Terahertz imaging of aircraft composites," English, Piscataway, NJ, USA, 2009, 2 pp. --.

[16] J. White, G Fichter, A Chernovsky, D. A. Zimdars, J. F. Whitaker, D Das, and T. M. Pollock, "Time-domain terahertz mapping of thickness and degradation of aircraft turbine blade thermal barrier coatings," in Conference on Lasers and Electro-Optics, Optical Society of America, 2008, CThN7.

[17] D. M. Mittleman, R. H. Jacobsen, and M. C. Nuss, "T-ray imaging," Selected Topics in Quantum Electronics, IEEE Journal of, vol. 2, no. 3, pp. 679-692, 1996.

[18] D. Mittleman, S. Hunsche, L. Boivin, and M. Nuss, "T-ray tomography," English, Optics Letters, vol. 22, no. 12, pp. 904 -6, 1997. 
[19] A. J. Fitzgerald, B. E. Cole, and P. F. Taday, "Nondestructive analysis of tablet coating thicknesses using terahertz pulsed imaging," Journal of Pharmaceutical Sciences, vol. 94, no. 1, pp. 177-183, 2005.

[20] T. Yasui, T. Yasuda, K. Sawanaka, and T. Araki, "Terahertz paintmeter for noncontact monitoring of thickness and drying progress in paint film," English, Applied Optics, vol. 44, no. 32, pp. 6849 -56, 2005.

[21] J. Takayanagi, H. Jinno, S. Ichino, K. Suizu, M. Yamashita, T. Ouchi, S. Kasai, H. Ohtake, H. Uchida, N. Nishizawa, and K. Kawase, "High-resolution timeof-flight terahertz tomography using a femtosecond fiber laser," Opt. Express, vol. 17, no. 9, pp. 7533-7539, 2009.

[22] K.-E. Peiponen, A. Zeitler, and M. Kuwata-Gonokami, Terahertz Spectroscopy and Imaging. Springer, 2013.

[23] S. C. Henry, L. M. Zurk, S. Schecklman, and D. D. Duncan, "Three-dimensional broadband terahertz synthetic aperture imaging," Optical Engineering, vol. 51, no. 9, pp. $091603-1,2012$.

[24] S. Henry, G. Kniffin, and L. Zurk, "3-d broadband terahertz synthetic aperture imaging," in 2012 37th International Conference on Infrared, Millimeter, and Terahertz Waves, 2012.

[25] L. Zurk, S. Henry, and S Schecklman, "Terahertz spectral imaging using correlation processing," in Infrared, Millimeter, and Terahertz Waves (IRMMWTHz), 2012 37th International Conference on, IEEE, 2012, pp. 1-2.

[26] M Awad, M Nagel, H Kurz, J Herfort, and K Ploog, "Characterization of low temperature gaas antenna array terahertz emitters," Applied Physics Letters, vol. 91, no. 18, p. $181124,2007$. 
[27] P. Hale, J. Madeo, C. Chin, S. Dhillon, J. Mangeney, J. Tignon, and K. Dani, "Broadband thz generation using interdigitated photoconductive antennas with a 15 fs, high power oscillator," in CLEO: Science and Innovations, Optical Society of America, 2013, CW3K-3.

[28] D. Saeedkia, M.-R. Esmaili-Rad, and M. Nagel, "Photoconductive aperture antenna arrays for generation and detection of terahertz radiation," in Infrared, Millimeter, and Terahertz Waves, 2009. IRMMW-THz 2009. 34th International Conference on, IEEE, 2009, pp. 1-2.

[29] P. Stoffa, J. Fokkema, R. de Luna Freire, and W. Kessinger, "Split-step fourier migration," English, Geophysics, vol. 55, no. 4, pp. $410-21,1990$.

[30] D. Lee, I. M. Mason, and G. M. Jackson, "Split-step fourier shot-record migration with deconvolution imaging," Geophysics, vol. 56, no. 11, pp. 1786-1793, 1991.

[31] R. H. Hardin and F. D. Tappert, "Applications of the split-step fourier method to the numerical solution of nonlinear and variable coefficient wave equation," SIAM Rev., vol. 15, 1973.

[32] F. B. Jensen, Computational ocean acoustics. Springer, 2011.

[33] F. J. Ryan, "Analysis of electromagnetic propagation over variable terrain using the parabolic wave equation," DTIC Document, Tech. Rep., 1991.

[34] J. R. Kuttler, "Differences between the narrow-angle and wide-angle propagators in the split-step fourier solution of the parabolic wave equation," Antennas and Propagation, IEEE Transactions on, vol. 47, no. 7, pp. 1131-1140, 1999. 
[35] A. R. Sena, P. L. Stoffa, and M. K. Sen, "Split step fourier migration of ground penetrating radar data," in 73rd Annual International Meeting, Society of Exploration Geophysicists, Expanded Abstracts, 2003, pp. 1023-1026.

[36] A. Sena, P. Stoffa, and M. Sen, "Split-step fourier migration of gpr data in lossy media," English, Geophysics, vol. 71, no. 4, pp. 77 -91, 2006.

[37] L. Huang and Y. Quan, "Ultrasound pulse-echo imaging using the split-step fourier propagator," in Medical Imaging, International Society for Optics and Photonics, 2007, pp. 651305-651305.

[38] H. Li, L. H. Le, M. D. Sacchi, and E. H. Lou, "Ultrasound imaging of long bone fractures and healing with the split-step fourier imaging method," Ultrasound in medicine $\& 3$ biology, vol. 39, no. 8, pp. 1482-1490, 2013.

[39] G. Kniffin, L. Zurk, S Schecklman, and S. Henry, "3d terahertz synthetic aperture imaging of objects with arbitrary boundaries," in SPIE Optical Engineering + Applications, International Society for Optics and Photonics, 2013, $88460 \mathrm{~T}-88460 \mathrm{~T}$.

[40] G. P. Kniffin and L. M. Zurk, "Terahertz non-destructive sensing of layered materials with curved surfaces," in Radar Symposium (IRS), 2015 16th International, IEEE, 2015, pp. 197-202.

[41] _ _ "Parabolic equation methods for terahertz 3d synthetic aperture imaging," Submitted for publication in IEEE Transactions on Terahertz Science and Technology, 2016.

[42] B. B. Hu and M. C. Nuss, "Imaging with terahertz waves," Opt. Lett., vol. 20, no. 16 , pp. 1716-1718, 1995. 
[43] C. M. Ciesla, D. D. Arnone, A. Corchia, D. A. Crawley, C. Longbottom, E. H. Linfield, and M. Pepper, Biomedical applications of terahertz pulse imaging, 2000.

[44] E Pickwell and V. P. Wallace, "Biomedical applications of terahertz technology," Journal of Physics D: Applied Physics, vol. 39, no. 17, R301, 2006.

[45] Y. Cai, Z. Wang, R. Dias, and D. Goyal, "Electro optical terahertz pulse reflectometryan innovative fault isolation tool," in Electronic Components and Technology Conference (ECTC), 2010 Proceedings 60th, IEEE, 2010, pp. 13091315.

[46] J. M. Chin, V. Narang, X. Zhao, M. Y. Tay, A. Phoa, V. Ravikumar, L. H. Ei, S. H. Lim, C. W. Teo, S. Zulkifli, M. C. Ong, and M. C. Tan, "Fault isolation in semiconductor product, process, physical and package failure analysis: importance and overview," Microelectronics Reliability, vol. 51, no. 9 - 11, pp. 1440 $-1448,2011$.

[47] C. Zandonella, "Terahertz imaging: t-ray specs," Nature, vol. 424, no. 6950, pp. $721-722,2003$.

[48] D. Zimdars, J. Valdmanis, J. S. White, G Stuk, S Williamson, W. P. Winfree, and E. I. Madaras, "Technology and applications of terahertz imaging non-destructive examination: inspection of space shuttle sprayed on foam insulation," in AIP Conference Proceedings, vol. 760, 2005, p. 570.

[49] A. Redo-Sanchez, N. Karpowicz, J. Xu, and X. Zhang, "Damage and defect inspection with terahertz waves," in International Workshop on Ultrasonic and Advanced Methods for Nondestructive Testing and Material Charact, 2006, pp. $67-78$. 
[50] D. Zimdars, J. S. White, G Stuk, A Chernovsky, G Fichter, and S Williamson, "Large area terahertz imaging and non-destructive evaluation applications," Insight-Non-Destructive Testing and Condition Monitoring, vol. 48, no. 9, pp. 537-539, 2006.

[51] N. Karpowicz, H. Zhong, C. Zhang, K.-I. Lin, J.-S. Hwang, J. Xu, and X.-C. Zhang, "Compact continuous-wave subterahertz system for inspection applications," Applied Physics Letters, vol. 86, no. 5, pp. 054 105-054 105, 2005.

[52] R. F. Anastasi and E. I. Madaras, "Application of hilbert-huang transform for improved defect detection in terahertz nde of shuttle tiles," in Nondestructive Evaulation for Health Monitoring and Diagnostics, International Society for Optics and Photonics, 2005, pp. 356-362.

[53] T. Lu, C. Snapp, T.-H. Chao, A. Thakoor, T. Bechtel, S. Ivashov, and I. Vasiliev, "Evaluation of holographic subsurface radar for nde of space shuttle thermal protection tiles," in Defense and Security Symposium, International Society for Optics and Photonics, 2007, 65550S-65550S.

[54] Y. Morita, A. Dobroiu, K. Kawase, and C. Otani, "Terahertz technique for detection of microleaks in the seal of flexible plastic packages," Optical Engineering, vol. 44, no. 1, pp. 019 001-019 001, 2005.

[55] Y. Morita, A. Dobroiu, C. Otani, and K. Kawase, "A real-time inspection system using a terahertz technique to detect microleak defects in the seal of flexible plastic packages," Journal of Food Protection®, vol. 68, no. 4, pp. 833$837,2005$.

[56] C Jördens, M Scheller, S Wietzke, D Romeike, C Jansen, T Zentgraf, K Wiesauer, V Reisecker, and M Koch, "Terahertz spectroscopy to study the 
orientation of glass fibres in reinforced plastics," Composites Science and Technology, vol. 70, no. 3, pp. 472-477, 2010.

[57] K. Fukunaga and M. Picollo, "Terahertz spectroscopy applied to the analysis of artists materials," Applied Physics A, vol. 100, no. 3, pp. 591-597, 2010.

[58] A. J. Adam, P. C. Planken, S. Meloni, and J. Dik, "Terahertz imaging of hidden paint layers on canvas," in Infrared, Millimeter, and Terahertz Waves, 2009. IRMMW-THz 2009. 34th International Conference on, IEEE, 2009, pp. 1-2.

[59] R. Groves, B Pradarutti, E Kouloumpi, W Osten, and G Notni, "2d and 3d non-destructive evaluation of a wooden panel painting using shearography and terahertz imaging," $N d t \&$ E International, vol. 42, no. 6, pp. 543-549, 2009.

[60] J. Jackson, M Mourou, J. Whitaker, I. Duling III, S. Williamson, M Menu, and G. Mourou, "Terahertz imaging for non-destructive evaluation of mural paintings," Optics Communications, vol. 281, no. 4, pp. 527-532, 2008.

[61] K Fukunaga, I Hosako, Y Kohdzuma, T Koezuka, M.-J. Kim, T Ikari, and X Du, "Terahertz analysis of an east asian historical mural painting," Journal of the European Optical Society-Rapid publications, vol. 5, 2010.

[62] J. A. Zeitler and L. F. Gladden, "In-vitro tomography and non-destructive imaging at depth of pharmaceutical solid dosage forms," European Journal of Pharmaceutics and Biopharmaceutics, vol. 71, no. 1, pp. 2-22, 2009.

[63] Y.-C. Shen, "Terahertz pulsed spectroscopy and imaging for pharmaceutical applications: a review," International journal of pharmaceutics, vol. 417, no. 1, pp. 48-60, 2011. 
[64] K. J. Siebert, H. Quast, R. Leonhardt, T. Loffler, M. Thomson, T. Bauer, H. G. Roskos, and S. Czasch, "Continuous-wave all-optoelectronic terahertz imaging," Applied Physics Letters, vol. 80, no. 16, pp. 3003-3005, 2002.

[65] N. Karpowicz, H. Zhong, J. Xu, K.-I. Lin, J.-S. Hwang, and X. Zhang, "Comparison between pulsed terahertz time-domain imaging and continuous wave terahertz imaging," Semiconductor Science and Technology, vol. 20, no. 7, S293, 2005.

[66] B. Ferguson, S. Wang, D. Gray, D. Abbot, and X.-C. Zhang, "T-ray computed tomography," Opt. Lett., vol. 27, no. 15, pp. 1312-1314, 2002.

[67] B. Ferguson, S. Wang, D. Gray, D. Abbott, and X. Zhang, "Towards functional 3d t-ray imaging," Physics in medicine and biology, vol. 47, p. 3735, 2002.

[68] B. Ferguson, S. Wang, D. Gray, D. Abbott, and X.-C. Zhang, "T-ray diffraction tomography," in OSA Trends in Optics and Photonics (TOPS), The Thirteenth International Conference on Ultrafast Phenomena, vol. 72, 2002, pp. $450-451$.

[69] S Wang, B Ferguson, D. Abbott, and X.-C. Zhang, "T-ray imaging and tomography," Journal of Biological Physics, vol. 29, no. 2-3, pp. 247-256, 2003.

[70] S. Wang and X.-C. Zhang, "Pulsed terahertz tomography," English, Journal of Physics D (Applied Physics), vol. 37, no. 4, pp. 1-36, 2004.

[71] M. Awad and R. Cheville, "Transmission terahertz waveguide-based imaging below the diffraction limit," Applied Physics Letters, vol. 86, no. 22, pp. $221107-221107,2005$. 
[72] K. L. Nguyen, M. L. Johns, L. Gladden, C. H. Worrall, P. Alexander, H. E. Beere, M. Pepper, D. A. Ritchie, J. Alton, S. Barbieri, et al., "Threedimensional imaging with a terahertz quantum cascade laser," Optics express, vol. 14, no. 6, pp. 2123-2129, 2006.

[73] A Brahm, M Bauer, T Hoyer, H Quast, T Loeffler, S Riehemann, G Notni, and A Tunnermann, "All-electronic 3d computed thz tomography," in Infrared, Millimeter and Terahertz Waves (IRMMW-THz), 2011 36th International Conference on, IEEE, 2011, pp. 1-2.

[74] W. Sun, X. Wang, Y. Cui, and Y. Zhang, "Continuous-wave terahertz tomography with phase unwrapping," in Lasers and Electro-Optics Europe (CLEO EUROPE/EQEC), 2011 Conference on and 12th European Quantum Electronics Conference, IEEE, 2011, pp. 1-1.

[75] N. Sunaguchi, Y. Sasaki, N. Maikusa, M. Kawai, T. Yuasa, and C. Otani, "Depth-resolving thz imaging with tomosynthesis," Opt. Express, vol. 17, no. 12, pp. 9558-9570, 2009.

[76] B. Ferguson and D. Abbott, "Wavelet de-noising of optical terahertz pulse imaging data," Fluctuation and Noise Letters, vol. 1, no. 02, pp. L65-L69, 2001.

[77] X. Yin, B. W.-H. Ng, B. Ferguson, and D. Abbott, "Wavelet based local tomographic image using terahertz techniques," Digital Signal Processing, vol. 19, no. 4, pp. 750-763, 2009.

[78] E. Abraham, A. Younus, C. Aguerre, P. Desbarats, and P. Mounaix, "Refraction losses in terahertz computed tomography," Optics Communications, vol. 283, no. 10, pp. 2050-2055, 2010. 
[79] S. Mukherjee, J. Federici, P. Lopes, and M. Cabral, "Elimination of fresnel reflection boundary effects and beam steering in pulsed terahertz computed tomography," Journal of Infrared, Millimeter, and Terahertz Waves, pp. 117, 2013.

[80] J. Pearce, H. Choi, D. Mittleman, J. White, and D. Zimdars, "Terahertz wide aperture reflection tomography," English, Optics Letters, vol. 30, no. 13, pp. $1653-5,2005$.

[81] C. Zhang, K. Mu, X. Jiang, Y. Jiao, L. Zhang, Q. Zhou, Y. Zhang, J. Shen, G. Zhao, and X. Zhang, "Identification of explosives and drugs and inspection of material defects with thz radiation," in Proc. of SPIE, vol. 6840, 2007, 68400S.

[82] H. L. Van Trees, Detection, Estimation, and Modulation Theory, Optimum Array Processing. John Wiley \& Sons, 2004.

[83] M. C. Sullivan, Practical Array Processing. McGraw-Hill, 2009.

[84] C. V. Jakowatz, D. E. Wahl, P. H. Eichel, D. C. Ghiglia, and P. A. Thompson, Spotlight-mode synthetic aperture radar: a signal processing approach. Kluwer Academic Publishers Norwell, MA, 1996, vol. 101.

[85] J. W. Goodman, Introduction to Fourier Optics, 3rd. Roberts \& Company, 2005.

[86] M. Dobrin and C. Savit, Introduction to geophysical prospecting, ser. McGrawHill International Editions: Geology series. McGraw-Hill Book Co., 1988.

[87] A. B. Ruffin, J. Decker, L. Sanchez-Palencia, L. Le Hors, J. F. Whitaker, T. B. Norris, and J. Rudd, "Time reversal and object reconstruction with singlecycle pulses," Optics Letters, vol. 26, no. 10, pp. 681-683, 2001. 
[88] A. B. Ruffin, J Van Rudd, J. Decker, L. Sanchez-Palencia, L. Le Hors, J. F. Whitaker, and T. B. Norris, "Time reversal terahertz imaging," Quantum Electronics, IEEE Journal of, vol. 38, no. 8, pp. 1110-1119, 2002.

[89] T. D. Dorney, J. L. Johnson, J Van Rudd, R. G. Baraniuk, W. W. Symes, and D. M. Mittleman, "Terahertz reflection imaging using kirchhoff migration," Optics letters, vol. 26, no. 19, pp. 1513-1515, 2001.

[90] J. O'Hara and D Grischkowsky, "Synthetic phased-array terahertz imaging," Optics letters, vol. 27, no. 12, pp. 1070-1072, 2002.

[91] Z. Zhang and T. Buma, "Adaptive terahertz imaging using a virtual transceiver and coherence weighting," Opt. Express, vol. 17, no. 20, pp. 17812-17817, 2009.

[92] _ _ " "Terahertz impulse imaging with sparse arrays and adaptive reconstruction," Selected Topics in Quantum Electronics, IEEE Journal of, vol. 17, no. 1, pp. 169-176, 2011.

[93] C. Passmann and H. Ermert, "A 100-mhz ultrasound imaging system for dermatologic and ophthalmologic diagnostics," Ultrasonics, Ferroelectrics and Frequency Control, IEEE Transactions on, vol. 43, no. 4, pp. 545-552, 1996.

[94] C. Frazier and W. O'Brien Jr, "Synthetic aperture imaging with a virtual source element," in Ultrasonics Symposium, 1996. Proceedings., 1996 IEEE, IEEE, vol. 2, 1996, pp. 1555-1558.

[95] C. H. Frazier and W. D. O'Brien Jr, "Synthetic aperture techniques with a virtual source element," Ultrasonics, Ferroelectrics and Frequency Control, IEEE Transactions on, vol. 45, no. 1, pp. 196-207, 1998. 
[96] K Mayer, R Marklein, K. Langenberg, and T Kreutter, "Three-dimensional imaging system based on fourier transform synthetic aperture focusing technique," Ultrasonics, vol. 28, no. 4, pp. 241-255, 1990.

[97] M. Schickert, M. Krause, and W. Müller, "Ultrasonic imaging of concrete elements using reconstruction by synthetic aperture focusing technique," Journal of Materials in Civil Engineering, vol. 15, no. 3, pp. 235-246, 2003.

[98] J. Case, J Robbins, S Kharkovsky, F Hepburn, and R. Zoughi, "Microwave and millimeter wave imaging of the space shuttle external fuel tank spray on foam insulation (sofi) using synthetic aperture focusing techniques (saft)," in AIP Conference Proceedings, vol. 820, 2006, p. 1546.

[99] S. Kharkovsky and R. Zoughi, "Microwave and millimeter wave nondestructive testing and evaluation-overview and recent advances," Instrumentation $\mathcal{E}$ Measurement Magazine, IEEE, vol. 10, no. 2, pp. 26-38, 2007.

[100] E. M. Johansson and J. E. Mast, "Three-dimensional ground penetrating radar imaging using synthetic aperture time-domain focusing," in Proc. of SPIE Conference on Advanced Microwave and Millimeter Wave Detectors, vol. 2275, 1994, pp. 205-214.

[101] L. Busse, "Three-dimensional imaging using a frequency-domain synthetic aperture focusing technique," Ultrasonics, Ferroelectrics and Frequency Control, IEEE Transactions on, vol. 39, no. 2, pp. 174-179, 1992.

[102] J. T. Ylitalo and H. Ermert, "Ultrasound synthetic aperture imaging: monostatic approach," Ultrasonics, Ferroelectrics and Frequency Control, IEEE Transactions on, vol. 41, no. 3, pp. 333-339, 1994. 
[103] M. H. Skjelvareid, T. Olofsson, Y. Birkelund, and Y. Larsen, "Synthetic aperture focusing of ultrasonic data from multilayered media using an omega-k algorithm," Ultrasonics, Ferroelectrics and Frequency Control, IEEE Transactions on, vol. 58, no. 5, pp. 1037-1048, 2011.

[104] M Leontovich and V Fock, "Solution of the problem of propagation of electromagnetic waves along the earths surface by the method of parabolic equation," Acad. Sci. USSR. J. Phys, vol. 10, pp. 13-24, 1946.

[105] V. A. Fock, Electromagnetic diffraction and propagation problems. Pergamon Press, 1965.

[106] D. Lee, A. D. Pierce, and E.-C. Shang, "Parabolic equation development in the twentieth century," Journal of Computational Acoustics, vol. 8, no. 04, pp. 527-637, 2000.

[107] D. Lee and S. McDaniel, Ocean Acoustic Propagation by Finite Difference Methods. New York: Pergamon, 1988.

[108] D. Huang, "Finite element solution to the parabolic wave equation," The Journal of the Acoustical Society of America, vol. 84, p. 1405, 1988.

[109] M. Feit and J. Fleck, "Light propagation in graded-index optical fibers," Applied optics, vol. 17, no. 24, pp. 3990-3998, 1978.

[110] M. D. Collins, "A split-step padé solution for the parabolic equation method," The Journal of the Acoustical Society of America, vol. 93, p. 1736, 1993.

[111] _ - "Generalization of the split-step padé solution," The Journal of the Acoustical Society of America, vol. 96, p. 382, 1994. 
[112] M. D. Collins, R. Cederberg, D. B. King, and S. A. Chin-Bing, "Comparison of algorithms for solving parabolic wave equations," The Journal of the Acoustical Society of America, vol. 100, p. 178, 1996.

[113] T. F. Duda, "Initial results from a cartesian three-dimensional parabolic equation acoustical propagation code," DTIC Document, Tech. Rep., 2006.

[114] Y.-T. Lin and T. F. Duda, "A higher-order split-step fourier parabolic-equation sound propagation solution scheme," The Journal of the Acoustical Society of America, vol. 132, no. 2, EL61-EL67, 2012.

[115] Y.-T. Lin, J. M. Collis, and T. F. Duda, "A three-dimensional parabolic equation model of sound propagation using higher-order operator splitting and padé approximants," The Journal of the Acoustical Society of America, vol. 132, no. 5, EL364-EL370, 2012.

[116] Y.-T. Lin, T. F. Duda, and A. E. Newhall, "Three-dimensional sound propagation models using the parabolic-equation approximation and the split-step fourier method," Journal of Computational Acoustics, vol. 21, no. 01, 2013.

[117] C. F. Bohren and D. R. Huffman, "Absorption and scattering by a sphere," Absorption and Scattering of Light by Small Particles, pp. 82-129, 1983.

[118] C. A. Balanis, Advanced Engineering Electromagnetics. John Wiley and Sons, 1989.

[119] A. L. Aden and M. Kerker, "Scattering of electromagnetic waves from two concentric spheres," Journal of Applied Physics, vol. 22, no. 10, pp. 1242$1246,1951$.

[120] G. T. Ruck, D. E. Barrick, W. D. Stuart, and C. K. Krichbaum, Radar cross section handbook. Plenum press New York, 1970, vol. 1. 
[121] C.-T. Tai, Dyadic Green functions in electromagnetic theory. IEEE press New York, 1994, vol. 272.

[122] N. L. Tsitsas and C. Athanasiadis, "On the scattering of spherical electromagnetic waves by a layered sphere," The Quarterly Journal of Mechanics and Applied Mathematics, vol. 59, no. 1, pp. 55-74, 2006.

[123] N. L. Tsitsas, "Direct and inverse dipole electromagnetic scattering by a piecewise homogeneous sphere," ZAMM-Journal of Applied Mathematics and Mechanics/Zeitschrift für Angewandte Mathematik und Mechanik, vol. 89, no. 10, pp. 833-849, 2009.

[124] W. J. Wiscombe, Mie scattering calculations: advances in technique and fast, vector-speed computer codes. Atmospheric Analysis and Prediction Division, National Center for Atmospheric Research, 1979.

[125] B. Johnson, "Light scattering by a multilayer sphere," Applied optics, vol. 35, no. 18, pp. 3286-3296, 1996.

[126] W. Yang, "Improved recursive algorithm for light scattering by a multilayered sphere," Applied optics, vol. 42, no. 9, pp. 1710-1720, 2003.

[127] O Peña and U Pal, "Scattering of electromagnetic radiation by a multilayered sphere," Computer Physics Communications, vol. 180, no. 11, pp. 2348-2354, 2009.

[128] A. Barnett, "The calculation of spherical bessel functions and coulomb functions," 1996.

[129] E Hernández, K Commeford, and M. Pérez-Quiles, "Matlab gui for computing bessel functions using continued fractions algorithm," Revista Brasileira de Ensino de Física, vol. 33, no. 1, pp. 01-08, 2011. 
[130] C. D. Cantrell et al., "Numerical methods for the accurate calculation of spherical bessel functions and the location of mie resonances," 2013.

[131] E. Wolf, "Three-dimensional structure determination of semi-transparent objects from holographic data," Optics Communications, vol. 1, no. 4, pp. 153$156,1969$.

[132] — "Determination of the amplitude and the phase of scattered fields by holography," JOSA, vol. 60, no. 1, pp. 18-20, 1970.

[133] R. Thomson, "Transverse and longitudinal resolution of the synthetic aperture focusing technique," Ultrasonics, vol. 22, no. 1, pp. 9-15, 1984.

[134] K. Langenberg, M Berger, T. Kreutter, K Mayer, and V Schmitz, "Synthetic aperture focusing technique signal processing," NDT international, vol. 19, no. 3, pp. 177-189, 1986.

[135] S. J. Norton and M. Linzer, "Ultrasonic reflectivity imaging in three dimensions: exact inverse scattering solutions for plane, cylindrical, and spherical apertures," Biomedical Engineering, IEEE Transactions on, no. 2, pp. 202$220,1981$.

[136] W. C. Chew, Waves and fields in inhomogenous media. IEEE press New York, 1995.

[137] R. Y. Chiao and L. J. Thomas, "Analytic evaluation of sampled aperture ultrasonic imaging techniques for nde," Ultrasonics, Ferroelectrics and Frequency Control, IEEE Transactions on, vol. 41, no. 4, pp. 484-493, 1994.

[138] C. V. Jakowatz Jr, D. E. Wahl, and D. A. Yocky, "Beamforming as a foundation for spotlight-mode sar image formation by backprojection," in SPIE 
Defense and Security Symposium, International Society for Optics and Photonics, 2008, 69700Q-69700Q.

[139] L.-J. Huang and M. C. Fehler, "Accuracy analysis of the split-step fourier propagator: implications for seismic modeling and migration," Bulletin of the Seismological Society of America, vol. 88, no. 1, pp. 18-29, 1998.

[140] D. W. Ricker, Echo signal processing. Springer, 2003.

[141] S. Schecklman, G. P. Kniffin, and L. M. Zurk, "Terahertz non-destructive evaluation of layered media with the maximum likelihood estimator," in $O p$ tomechatronic Technologies (ISOT), 2014 International Symposium on, IEEE, 2014, pp. 81-85.

[142] B. Haider, P. A. Lewin, and K. E. Thomenius, "Pulse elongation and deconvolution filtering for medical ultrasonic imaging," Ultrasonics, Ferroelectrics and Frequency Control, IEEE Transactions on, vol. 45, no. 1, pp. 98-113, 1998.

[143] M. D. Collins, "Users guide for ram versions 1.0 and 1.0 p," Naval Research Lab, Washington, DC, vol. 20375, 1995.

[144] R. A. Zingarelli and D. King, Ram to navy standard parabolic equation: transition from research to fleet acoustic model, 2003. 


\section{Appendix A}

\section{Mie Series Model for Scattering from a Layered Dielectric Sphere}

This appendix describes work conducted in the implementation and testing of a Mie series model for the electromagnetic scattering from a layered dielectric sphere, which was originally intended to serve as a source of simulation data for testing the ability of the split-step Fourier method from Sec. 3.2.2 to accurately quantify the thickness of a curved dielectric layer. However, the analysis in Sec. 4.3 proved to be more useful for this purpose. Nonetheless, the work in Mie series scattering from a layered dielectric sphere represents a significant body of the work completed as part of this research and is therefore included in hopes that it may assist in future work in this area.

\section{A.1 Mie Series Model Derivation}

The basic concept of the Mie formulation of electromagnetic scattering from a sphere is to decompose the fields into a sum of spherical harmonics, each of which are weighted by a corresponding Mie coefficient. Mie coefficients are obtained by enforcing the boundary conditions, i.e. by ensuring that the tangential components of the electric and magnetic fields are continuous across the boundaries between media. This 


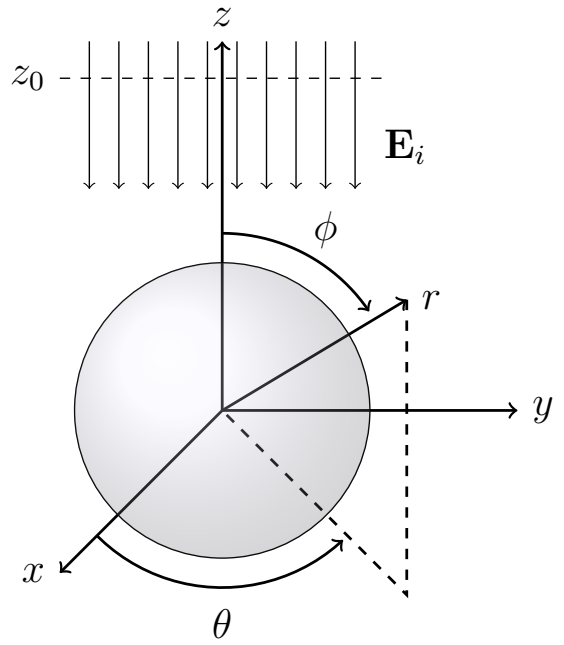

Figure A.1.1: Dielectric sphere illuminated by a plane wave propagating in the $-z$ direction, whose phase is 0 in the $z_{0}$ plane.

appendix gives an overview of the methods used in implementing the Mie series calculations in Matrab. The formulation is based on references [125-127].

For the unit-amplitude, $x$-polarized plane wave $\mathbf{E}_{i}$ with zero phase at $z=0$ illustrated in Fig. A.1.1, the Mie series expansion is given by $[117,125]$

$$
\begin{aligned}
\mathbf{E}_{i} & =\hat{\mathbf{x}} e^{i k\left(z-z_{0}\right)} \\
& =e^{-i k z_{0}} \sum_{m=1}^{\infty} \frac{2 m+1}{m(m+1)} i^{m}\left(\mathbf{M}_{o 1 m}^{(1)}-i \mathbf{N}_{e 1 m}^{(1)}\right)
\end{aligned}
$$

Similarly, the scattered electric fields are given by

$$
\mathbf{E}_{s}=e^{-i k z_{0}} \sum_{m=1}^{\infty} \frac{2 m+1}{m(m+1)} i^{m}\left(a_{m} \mathbf{M}_{o 1 m}^{(3)}-i b_{m} \mathbf{N}_{e 1 m}^{(3)}\right)
$$

Outside the sphere, the total electric fields are given by the sum of the incident and scattered fields,

$$
\mathbf{E}=\mathbf{E}_{i}+\mathbf{E}_{s}
$$




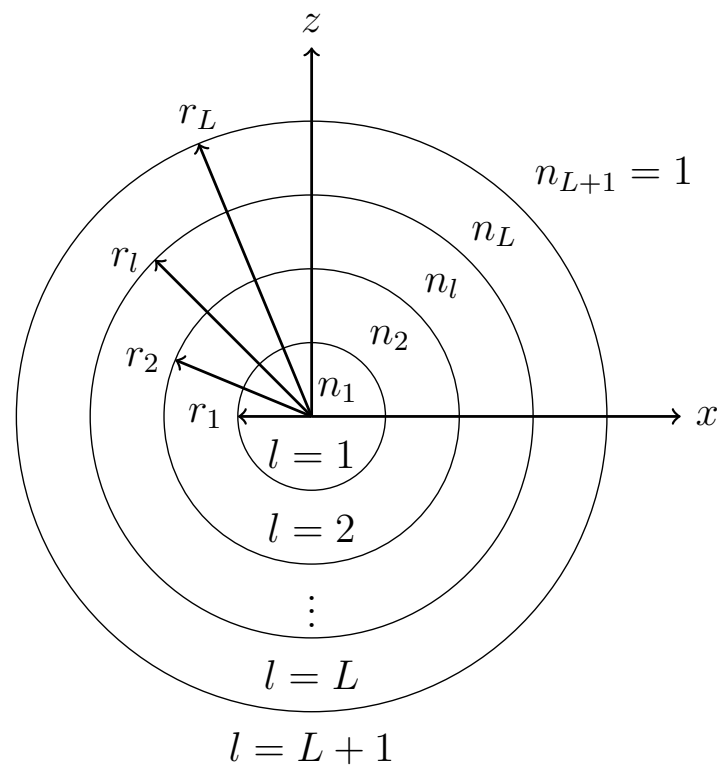

Figure A.1.2: L-layered dielectric sphere. Each layer has outer radius $r_{l}$ and refractive index $n_{l}$. Outside the sphere, $l=L+1$ and $n_{L+1}=1$ (free space).

while inside the sphere - which is assumed to be composed of $L$ layers of dielectric material as shown in Fig. A.1.2 - the total electric and magnetic fields in the $l^{\text {th }}$ layer are given by

$\mathbf{E}_{l}=e^{-i k z_{0}} \sum_{m=1}^{\infty} \frac{2 m+1}{m(m+1)} i^{m}\left[A_{m}^{(l)}\left(\mathbf{M}_{o 1 m}^{(1)}+a_{m}^{(l)} \mathbf{M}_{o 1 m}^{(3)}\right)-i B_{m}^{(l)}\left(\mathbf{N}_{e 1 m}^{(1)}+b_{m}^{(l)} \mathbf{N}_{e 1 m}^{(3)}\right)\right]$

$\mathbf{H}_{l}=-\frac{k e^{-i k z_{0}}}{\omega \mu_{0}} \sum_{m=1}^{\infty} \frac{2 m+1}{m(m+1)} i^{m}\left[B_{m}^{(l)}\left(\mathbf{M}_{e 1 m}^{(1)}+b_{m}^{(l)} \mathbf{M}_{e 1 m}^{(3)}\right)+i A_{m}^{(l)}\left(\mathbf{N}_{o 1 m}^{(1)}+a_{m}^{(l)} \mathbf{N}_{o 1 m}^{(3)}\right)\right]$

where $A_{m}^{(l)}, B_{m}^{(l)}, a_{m}^{(l)}$, and $b_{m}^{(l)}$ are the Mie coefficients in the $l^{\text {th }}$ layer and the vector 
spherical harmonics are given by [117]

$$
\begin{aligned}
\mathbf{M}_{o 1 m}= & \cos \phi \pi_{m}(\theta) z_{m}(n k r) \hat{\boldsymbol{\theta}}-\sin \phi \tau_{m}(\theta) z_{m}(n k r) \hat{\boldsymbol{\phi}}, \\
\mathbf{M}_{e 1 m}= & -\sin \phi \pi_{m}(\theta) z_{m}(n k r) \hat{\boldsymbol{\theta}}-\cos \phi \tau_{m}(\theta) z_{m}(n k r) \hat{\boldsymbol{\phi}} \\
\mathbf{N}_{o 1 m}= & \sin \phi m(m+1) \sin \theta \pi_{m}(\theta) \frac{z_{m}(n k r)}{n k r} \hat{\mathbf{r}} \\
& \quad+\sin \phi \tau_{m}(\theta) \frac{\hat{z}_{m}^{\prime}(n k r)}{n k r} \hat{\boldsymbol{\theta}}+\cos \phi \pi_{m}(\theta) \frac{\hat{z}_{m}^{\prime}(n k r)}{n k r} \hat{\boldsymbol{\phi}} \\
& \quad \cos \phi m(m+1) \sin \theta \pi_{m}(\theta) \frac{z_{m}(n k r)}{n k r} \hat{\mathbf{r}} \\
& \quad+\cos \phi \tau_{m}(\theta) \frac{\hat{z}_{m}^{\prime}(n k r)}{n k r} \hat{\boldsymbol{\theta}}-\sin \phi \pi_{m}(\theta) \frac{\hat{z}_{m}^{\prime}(n k r)}{n k r} \hat{\boldsymbol{\phi}} .
\end{aligned}
$$

The superscripts applied to the spherical harmonics in (A.1.4) indicate the type of spherical Bessel function represented by $z_{m}(\rho)$ in (A.1.5), i.e.

$$
z_{m}(\rho)= \begin{cases}j_{m}(\rho) & \text { for } \mathbf{M}^{(1)} \text { and } \mathbf{N}^{(1)} \\ h_{m}^{(1)}(\rho) & \text { for } \mathbf{M}^{(3)} \text { and } \mathbf{N}^{(3)}\end{cases}
$$

In addition, the Riccati-Bessel functions and their derivatives are given by

$$
\begin{aligned}
& \hat{z}_{m}(\rho)=\rho z_{m}(\rho), \\
& \hat{z}_{m}^{\prime}(\rho)=\frac{\partial}{\partial \rho} \hat{z}_{m}(\rho) .
\end{aligned}
$$

Substituting (A.1.5) into (A.1.4a) and (A.1.4b) yields the $r, \theta$, and $\phi$ components of 
the electric fields [125],

$$
\begin{aligned}
& E_{r}=-i e^{-i k z_{0}} \frac{\cos \phi \sin \theta}{[n(r) k r]^{2}} \sum_{m=1}^{\infty} i^{m}(2 m+1) T_{m}(r) \pi_{m}(\theta) \\
& E_{\theta}=e^{-i k z_{0}} \frac{\cos \phi}{k r} \sum_{m=1}^{\infty} i^{m} \frac{2 m+1}{m(m+1)}\left[S_{m}(r) \pi_{m}(\theta)-\frac{i}{k n^{2}(r)} T_{m}^{\prime}(r) \tau_{m}(\theta)\right] \\
& E_{\phi}=-e^{-i k z_{0}} \frac{\sin \phi}{k r} \sum_{m=1}^{\infty} i^{m} \frac{2 m+1}{m(m+1)}\left[S_{m}(r) \tau_{m}(\theta)-\frac{i}{k n^{2}(r)} T_{m}^{\prime}(r) \pi_{m}(\theta)\right],
\end{aligned}
$$

and magnetic fields [125],

$$
\begin{aligned}
& H_{r}=-i \frac{k e^{-i k z_{0}}}{\omega \mu_{0}} \frac{\sin \phi \sin \theta}{(k r)^{2}} \sum_{m=1}^{\infty} i^{m}(2 m+1) S_{m}(r) \pi_{m}(\theta) \\
& H_{\theta}=\frac{k e^{-i k z_{0}}}{\omega \mu_{0}} \frac{\sin \phi}{k r} \sum_{m=1}^{\infty} i^{m} \frac{2 m+1}{m(m+1)}\left[T_{m}(r) \pi_{m}(\theta)-\frac{i}{k} S_{m}^{\prime}(r) \tau_{m}(\theta)\right] \\
& H_{\phi}=\frac{k e^{-i k z_{0}}}{\omega \mu_{0}} \frac{\cos \phi}{k r} \sum_{m=1}^{\infty} i^{m} \frac{2 m+1}{m(m+1)}\left[T_{m}(r) \tau_{m}(\theta)-\frac{i}{k} S_{m}^{\prime}(r) \pi_{m}(\theta)\right]
\end{aligned}
$$

where the $\pi_{m}$ and $\tau_{m}$ terms are given by

$$
\begin{aligned}
\pi_{m}(\theta) & =\frac{1}{\sin \theta} P_{m}^{1}(\cos \theta) \\
\tau_{m}(\theta) & =\frac{d}{d \theta} P_{m}^{1}(\cos \theta)
\end{aligned}
$$

and $P_{m}^{1}(\cos \theta)$ is the associated Legendre function of order 1 and degree $m$. The radial dependence in (A.1.9) and (A.1.10) is given by the Debye potentials $S_{m}(r)$ and $T_{m}(r)$, their radial derivatives $S_{m}^{\prime}(r)$ and $T_{m}^{\prime}(r)$, and the radially-varying refractive index 
$n(r)$. In the $l^{\text {th }}$ layer, the Debye potentials and their derivatives are given by

$$
\begin{aligned}
S_{m}^{(l)}(r) & =A_{m}^{(l)}\left[\hat{j}_{m}\left(n_{l} k r\right)+a_{m}^{(l)} \hat{h}_{m}\left(n_{l} k r\right)\right], \\
T_{m}^{(l)}(r) & =B_{m}^{(l)}\left[\hat{j}_{m}\left(n_{l} k r\right)+b_{m}^{(l)} \hat{h}_{m}\left(n_{l} k r\right)\right],
\end{aligned}
$$

and

$$
\begin{aligned}
S_{m}^{(l)}(r) & \equiv \frac{\partial}{\partial r} S_{m}^{(l)}(r), \\
T_{m}^{(l)}(r) & \equiv \frac{\partial}{\partial r} T_{m}^{(l)}(r) .
\end{aligned}
$$

Given that the tangential $(\theta$ and $\phi)$ components must be continuous across the boundaries, (A.1.9b) and (A.1.9c) imply that $S_{m}(r)$ and $T_{m}^{\prime}(r) / n^{2}(r)$ must also be continuous across the boundaries. Likewise, (A.1.10b) and (A.1.10c) imply that $T_{m}(r)$ and $S_{m}^{\prime}(r)$ must also be continuous across the boundaries. The Mie coefficients are solved by enforcing these continuity conditions, starting with the logarithmic derivatives of the Riccati-Bessel functions

$$
\begin{aligned}
& D_{m}^{(1)}(\rho) \equiv \frac{\hat{j}_{m}^{\prime}(\rho)}{\hat{j}_{m}(\rho)}, \\
& D_{m}^{(2)}(\rho) \equiv \frac{\hat{h}_{m}^{\prime}(\rho)}{\hat{h}_{m}(\rho)},
\end{aligned}
$$

and the ratio

$$
R_{m}(\rho) \equiv \frac{\hat{j}_{m}(\rho)}{\hat{h}_{m}(\rho)} .
$$

Calculations of (A.1.16), (A.1.17), and (A.1.18) are more efficient and numerically stable than that of the Riccati-Bessel functions or their derivatives themselves [124- 
126] and can be easily implemented using the methods in [127]. Taking the ratios of the continuous terms scaled by $1 / k$ yields the modified logarithmic derivatives of the Debye potentials [125],

$$
\begin{aligned}
H_{m}^{a}(r) & =\frac{1}{k} \frac{S_{m}^{\prime}(r)}{S_{m}(r)} \\
H_{m}^{b}(r) & =\frac{1}{k n^{2}(r)} \frac{T_{m}^{\prime}(r)}{T_{m}(r)},
\end{aligned}
$$

which at just inside of the $l^{\text {th }}$ boundary (in the $l^{\text {th }}$ region) can be rewritten in terms of $R_{m}\left(n_{l} k r_{l}\right), D_{m}^{(1)}\left(n_{l} k r_{l}\right)$, and $D_{m}^{(2)}\left(n_{l} k r_{l}\right)$ as [125]

$$
\begin{aligned}
H_{m}^{a}\left(r_{l}\right) & =n_{l} \frac{R_{m}\left(n_{l} k r_{l}\right) D_{m}^{(1)}\left(n_{l} k r_{l}\right)+a_{m}^{(l)} D_{m}^{(2)}\left(n_{l} k r_{l}\right)}{R_{m}\left(n_{l} k r_{l}\right)+a_{m}^{(l)}}, \\
H_{m}^{b}\left(r_{l}\right) & =\frac{1}{n_{l}} \frac{R_{m}\left(n_{l} k r_{l}\right) D_{m}^{(1)}\left(n_{l} k r_{l}\right)+b_{m}^{(l)} D_{m}^{(2)}\left(n_{l} k r_{l}\right)}{R_{m}\left(n_{l} k r_{l}\right)+b_{m}^{(l)}}
\end{aligned}
$$

As these expressions are the same just outside the $l^{\text {th }}$ boundary (in the $l+1^{\text {th }}$ region), they can be set equal to one another across the boundary and solved for $a_{n}^{(l+1)}$ and $b_{n}^{(l+1)}$, resulting in [125]

$$
\begin{aligned}
& a_{m}^{(l+1)}=-R_{m}\left(n_{l+1} k r_{l}\right) \frac{H_{m}^{a}\left(n_{l} k r_{l}\right)-n_{l+1} D_{m}^{(1)}\left(n_{l+1} k r_{l}\right)}{H_{m}^{a}\left(n_{l} k r_{l}\right)-n_{l+1} D_{m}^{(2)}\left(n_{l+1} k r_{l}\right)}, \\
& b_{m}^{(l+1)}=-R_{m}\left(n_{l+1} k r_{l}\right) \frac{n_{l+1} H_{m}^{b}\left(n_{l} k r_{l}\right)-D_{m}^{(1)}\left(n_{l+1} k r_{l}\right)}{n_{l+1} H_{m}^{b}\left(n_{l} k r_{l}\right)-D_{m}^{(2)}\left(n_{l+1} k r_{l}\right)} .
\end{aligned}
$$

Using these terms, the Mie coefficients for each layer can be determined recursively, starting with the innermost layer, where $l=1$ and

$$
a_{m}^{(1)}=b_{m}^{(1)}=0
$$


which zeros out the influence of the spherical Hankel functions, whose imaginary parts go to $-\infty$ at the origin [125-127]. From (A.1.21) and (A.1.22), this also results in

$$
\begin{aligned}
& H_{m}^{a}\left(n_{1} k r_{1}\right)=n_{1} D_{m}^{(1)}\left(n_{1} k r_{1}\right), \\
& H_{m}^{b}\left(n_{1} k r_{1}\right)=\frac{1}{n_{1}} D_{m}^{(1)}\left(n_{1} k r_{1}\right) .
\end{aligned}
$$

Once the Mie coefficients $a_{m}^{(L+1)}$ and $b_{m}^{(L+1)}$ in the background medium have been determined, the scattered electric fields can be calculated by substituting $a_{m}=a_{m}^{(L+1)}$ and $b_{m}=b_{m}^{(L+1)}$ into (A.1.2).

\section{A.2 Mie Series Model Validation}

This section outlines numerical tests of the MATLAB implementation described in the previous section conducted to verify that the code is generating results comparable to published results from the literature.

Two publications in particular, [127] and [125], provide useful results for validating sphere model calculations described in Sec. A.1. The first reference, a 2009 paper by Peña and Pal [127], presents an algorithm for calculating the Mie coefficients $a_{m}$ and $b_{m}$ for a layered dielectric sphere, which are then used to calculate the extinction cross section $C_{\text {ext }}$, scattering cross section $C_{s c a}$, and albedo $A$ as a function of the size 
Table A.2.1: Refractive indices and fractional volumes used in calculations for 5layered sphere shown in Fig. A.2.1 [127].

\begin{tabular}{|c||c|c|c|c|c|}
\hline Layer, $l$ & 1 & 2 & 3 & 4 & 5 \\
\hline $\begin{array}{c}\text { Refractive Index, } \\
n_{l}\end{array}$ & $1.8+\mathrm{i} 1.7$ & $0.8+\mathrm{i} 0.7$ & $1.2+\mathrm{i} 0.09$ & $2.8+\mathrm{i} 0.2$ & $1.5+\mathrm{i} 0.4$ \\
\hline $\begin{array}{c}\text { Fractional Volume, } \\
V_{l} / V_{\text {total }}\end{array}$ & 0.1 & 0.26 & 0.044 & 0.3666 & 0.2294 \\
\hline
\end{tabular}

parameter $k r_{L}$ using

$$
\begin{aligned}
C_{e x t} & =\frac{2}{\left(k r_{L}\right)^{2}} \sum_{m=1}^{\infty}(2 m+1) \operatorname{Re}\left(a_{m}+b_{m}\right), \\
C_{s c a} & =\frac{2}{\left(k r_{L}\right)^{2}} \sum_{m=1}^{\infty}(2 m+1)\left(\left|a_{m}\right|^{2}+\left|b_{m}\right|^{2}\right), \\
A & =\frac{C_{s c a}}{C_{\text {ext }}} .
\end{aligned}
$$

The paper also provides an implementation of these calculations in ANSI C, which allows a convenient means of comparing the results side-by-side with those from the Matlab implementation of the algorithm in Sec. A.1. One benchmark calculation from [127] is the case of a lossy 5-layered sphere characterized by refractive indices and fractional volumes summarized in Table A.2.1. The calculated cross sections and albedo are plotted in Fig. A.2.1 as a function of the size parameter using both implementations. The results are shown to be in close agreement.

As SA imaging requires coherent field measurements rather than just the scattering cross sections from (A.2.3), simulations of SA data require coherent calculations of the scattered electric fields. One benchmark for these calculations is provided in a 1996 paper by B. R. Johnson [125] for the case of a Luneburg lens, which has a 


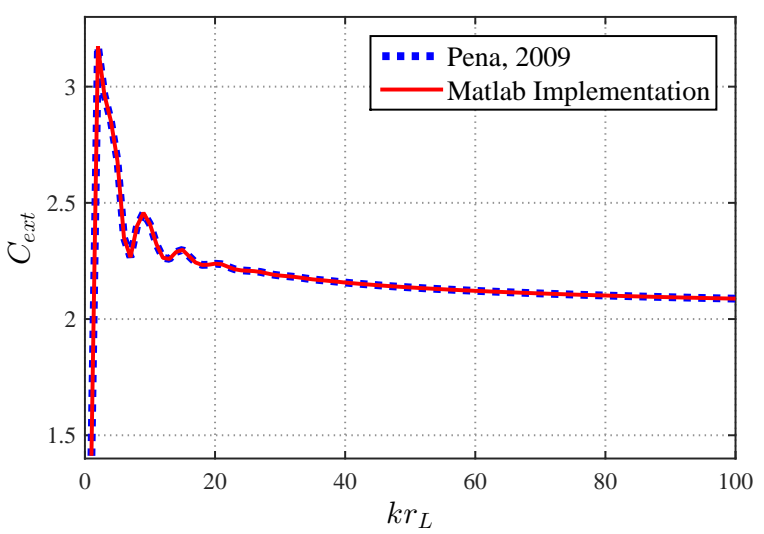

(a)

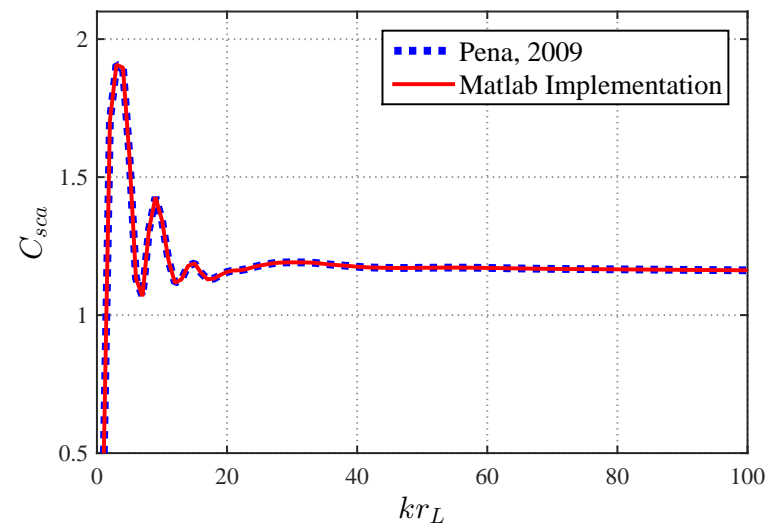

(b)

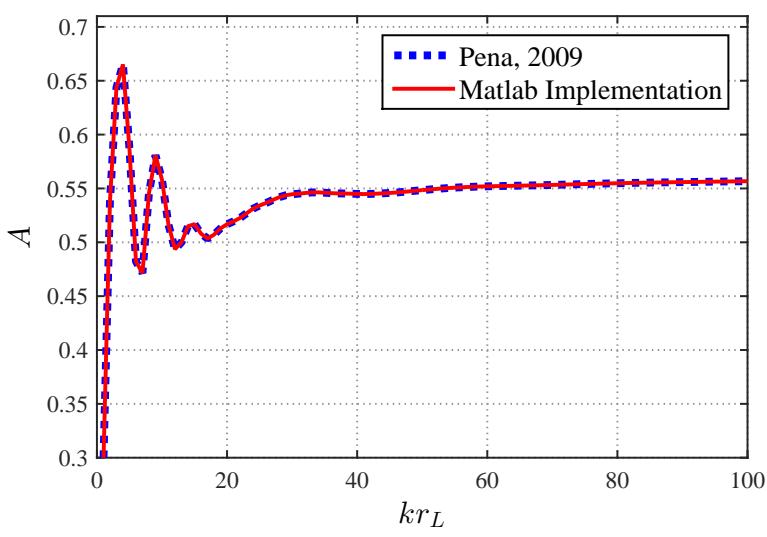

(c)

Figure A.2.1: Comparison of (a) extinction cross section $C_{\text {ext }}$, (b) scattering cross section $C_{s c a}$, and (c) albedo $A$ as a function of size parameter $k r_{L}$ using (A.2.3) from Mie coefficients $a_{m}$ and $b_{m}$ calculated using ANSI C code from Peña, 2009 [127] and MatLab implementation of layered sphere model described in Sec. A.1. 


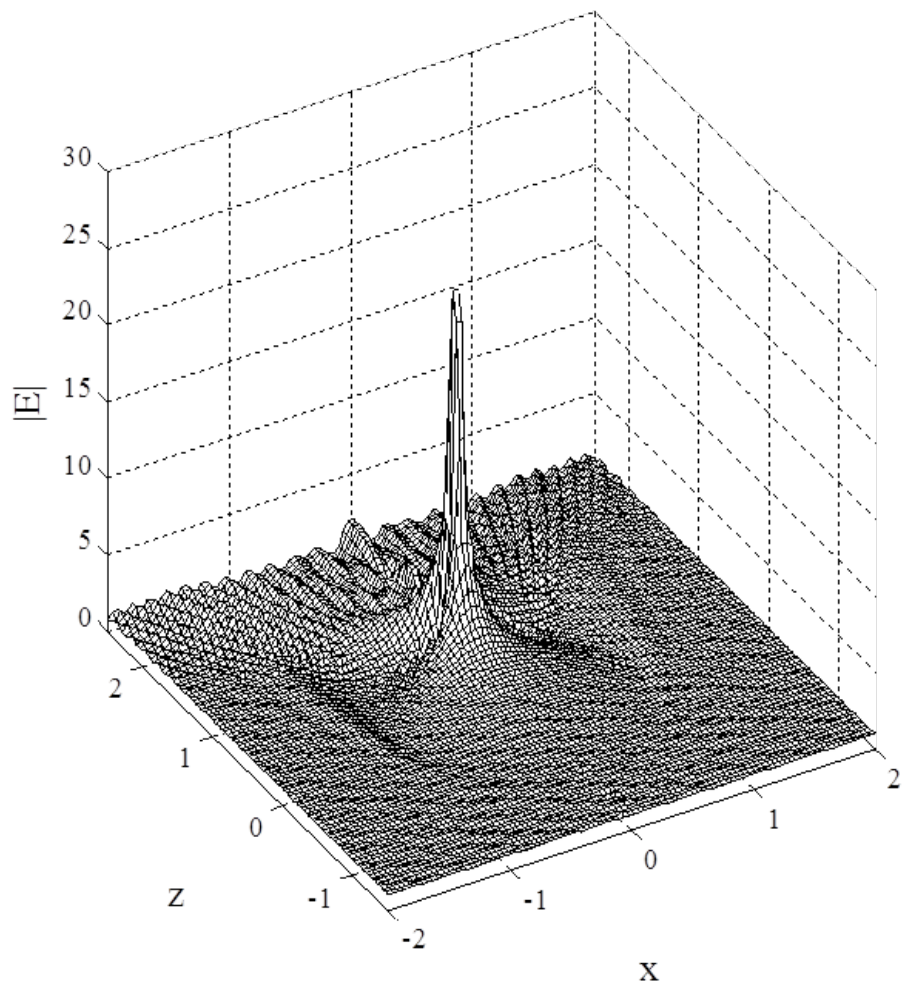

Figure A.2.2: Internal and external fields calculated using MATLAB implementation of Mie series calculations described in Sec. A.1 for validation against Fig. 7 of [125].

radially-varying refractive index profile given by

$$
n(r)=\sqrt{2-\left(\frac{r}{a}\right)^{2}} .
$$

This refractive index profile acts to focus an incident plane wave to a point on the back surface of the spherical lens. Fig. 7 in [125] gives a surface plot of the electric field amplitude both inside and outside of the Luneburg lens. The simulation was carried out with the refractive index in (A.2.4) approximated as a 500-layer sphere with size parameter $k a=60$. These calculations were duplicated using the MATLAB implementation for comparison, the results of which are shown in Fig. A.2.2. Fig. 7 in $[125]$ is nearly identical to the result in the figure, with the prominent $30 \mathrm{~V} / \mathrm{m}$ amplitude spike occurring in the same place for both results. Also, the electric field 
"ripples" are similarly behaved, indicting the MATLAB implementation from Sec. A.1 is operating consistently with the published results from [125]. 